O problema de Kepler, uma solução coreográfica para

o problema de três corpos e alguns resultados sobre configurações centrais

Antonio Carlos Fernandes

\author{
DISSERTAÇÃO APRESENTADA \\ AO \\ Instituto de Matemática e Estatística \\ DA \\ Universidade de SÃo Paulo \\ PARA \\ OBTENÇÃO DO TÍTULO \\ $\mathrm{DE}$ \\ Mestre em CiênCIAS
}

Programa: Matemática Aplicada

Orientador: Prof. Dr. Clodoaldo Grotta Ragazzo

Durante o desenvolvimento deste trabalho o autor recebeu auxílio financeiro da FAPESP

Bolsa 2007/58037-6

São Paulo, Julho de 2009 


\title{
O problema de Kepler, uma solução coreográfica para \\ o problema de três corpos e alguns resultados sobre configurações centrais
}

\author{
Este exemplar corresponde à redação \\ final da dissertação devidamente corrigida \\ e defendida por (Antonio Carlos Fernandes) \\ e aprovada pela Comissão Julgadora.
}

Banca Examinadora:

- Prof. Dr. Clodoaldo Grotta Ragazzo(orientador) - IME-USP.

- Prof. Dr. Luis Fernando de Osório Mello - DMC-UNIFEI.

- Prof. Dr. Eduardo Shirlipe Goes Leandro - DMAT-UFPE. 


\section{Agradecimentos}

Primeiramente, agradeço muito ao Professor Clodoaldo pela amizade, orientação e pelos ensinamentos sobre Mecânica.

Agradeço ao Professor Luis Fernando pela motivação matemática de algumas das idéias aqui colocadas.

Agradeço à Tatiane e ao Guilherme, por estarem junto a mim nesta empreitada.

Por fim, agradeço aos meus familiares e amigos que direta ou indiretamente contribuíram para a realização deste trabalho. 


\section{Resumo}

No presente trabalho apresentamos algumas soluções clássicas para o problema de dois e três corpos. Uma solução memorável para o problema de três corpos, na qual os corpos perfazem uma coreografia em forma de Oito, esta solução foi encontrada em 2000 por Montgomery e Chenciner. Por fim abordamos um pouco do problema clássico de $n$-Corpos e suas soluções particulares.

Palavras-chave: Problema de Kepler, Solução Coreográfica, Configuração Central. 


\section{Abstract}

In this work we present some classical solutions for the Newtonian problem with two and three bodies. A remarkable solution for the three body problem, found by Montgomery and Chenciner, in which each body has an eight-shaped orbit is presented. Finally some particular solutions of the $n$-body problem and the so called central configurations, are presented and discussed.

Keywords: Kepler problem, Choreographic Solution, Central Configurations. 


\section{Sumário}

$\begin{array}{ll}\text { Lista de Figuras } & \text { ix }\end{array}$

1 Introdução $\quad 1$

1.1 Noções Preliminares da Mecânica Newtoniana . . . . . . . . . . . . . . . . . . . 4

1.2 Noções Preliminares da Mecânica Lagrangiana . . . . . . . . . . . . . . . . . 7

2 O Problema de Kepler $\quad 11$

2.1 Leis de Kepler e a Gravitação de Newton . . . . . . . . . . . . . . . . . . . . . 12

2.2 Alguns Aspectos Geométricos do Problema de Kepler . . . . . . . . . . . . . . . . 22

2.3 Regularização . . . . . . . . . . . . . . . . . . . . . . . 27

3 Problemas de Três Corpos $\quad 39$

3.1 Problema Newtoniano de 3-corpos . . . . . . . . . . . . . . . . . . 39

3.2 Uma Solução Memorável para o Problema de Três Corpos . . . . . . . . . . . . . . 48

3.2.1 Construção do Espaço de Triângulos Orientados. . . . . . . . . . . . . . . . . 48

3.2.2 A Construção da Órbita em Forma de "Oito". . . . . . . . . . . . . . . . 57

3.2 .3 A Órbita em Oito . . . . . . . . . . . . . . . . . . . . 59

4 O Problema de $n$-Corpos $\quad 71$

4.1 Não-Integrabilidade do Problema de $n$-corpos . . . . . . . . . . . . . . . 73

4.2 Alguns resultados do problema geral de $n$-corpos . . . . . . . . . . . . . 74

5 Configurações Centrais $\quad \mathbf{8 1}$

5.1 Definições e Resultados da Literatura . . . . . . . . . . . . . . . . . . 81

5.2 Resultados . . . . . . . . . . . . . . . . . . . 85

Referências Bibliográficas 111

$\begin{array}{lr}\text { Índice Remissivo } & 114\end{array}$ 


\section{Lista de Figuras}

1.1 Representação da trajetória epicicloidal de um planeta no modelo de Ptolomeu. . . . 2

1.2 Representação do sistema solar segundo Copérnico [6] . . . . . . . . . . . . . . 2

2.1 Plano de movimento onde evidenciamos o uso de coordenadas polares. . . . . . . . . 14

2.2 Possíveis movimentos para $J \neq 0 \ldots \ldots \ldots \ldots \ldots \ldots \ldots$

2.3 Órbita em forma de elipse. . . . . . . . . . . . . . . . . . . 18

2.4 Lei das áreas $A_{1}=A_{2} \ldots \ldots \ldots \ldots \ldots \ldots \ldots \ldots$

2.5 Hodógrafo do caso $E<0$, note que o círculo de velocidades é completamente percorrido. 26

2.6 Hodógrafo do caso $E>0$, note que o círculo de velocidades não é completamente

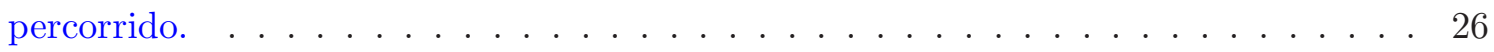

2.7 Hodógrafo do caso $E=0$, note que o círculo de velocidades é percorrido a menos de

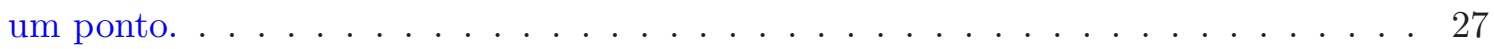

2.8 Movimento regularizado para os vários valores de energia. . . . . . . . . . . . . . 29

2.9 Projeção estereográfica para o caso $n=2 \ldots \ldots \ldots \ldots \ldots$

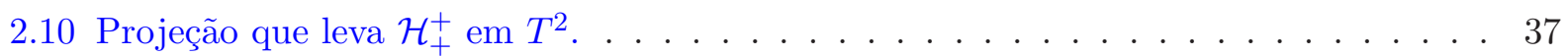

3.1 Representação das coordenadas relativas. . . . . . . . . . . . . . . . . . . 41

3.2 Representação da solução de equilíbrio relativo de Euler. Aqui $m_{1}: m_{2}: m_{3}=2: 3: 4$. 44

3.3 Representação da solução de Euler. Aqui $m_{1}: m_{2}: m_{3}=2: 3: 4 \ldots \ldots 4$

3.4 Representação da solução de equilíbrio relativo de Lagrange. Aqui devemos ter

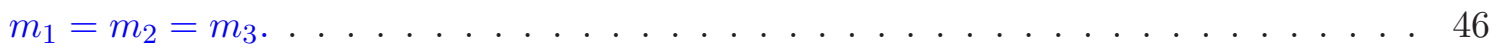

3.5 Representação da solução de Lagrange. Aqui devemos ter $m_{1}<m_{2}<m_{3}$. . . . . 47

3.6 A Órbita em oito de Chenciner e Montgomery. . . . . . . . . . . . . . . . . . . 48

3.7 Triângulo $\mathcal{T}$ formado pelos três corpos. . . . . . . . . . . . . . . . . . . . . 49

3.8 Coordenadas e ângulos em $\mathcal{T} \ldots \ldots \ldots \ldots$

3.9 Plano equivalente ao momento de inércia unitário. . . . . . . . . . . . . . 51

3.10 Representação de alguns pontos no plano. . . . . . . . . . . . . . . . 53

3.11 Ilustração da retirada do disco de $a+b+c=3 \ldots \ldots \ldots \ldots$. . . . . . . . 54

3.12 Espaço dos triângulos orientados. . . . . . . . . . . . . . . . . . 55 
3.13 Representação da aplicação de Hopf, note que a esfera à esquerda está em $\mathbb{C}^{2} \ldots$. . 57

3.14 A curva do Oito sobre a esfera de forma. . . . . . . . . . . . . . . . . 58

3.15 Movimento dos corpos sobre o Oito. . . . . . . . . . . . . . 59

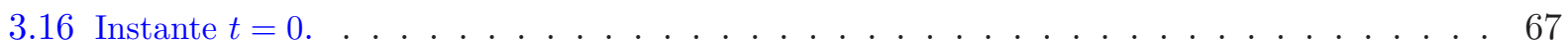

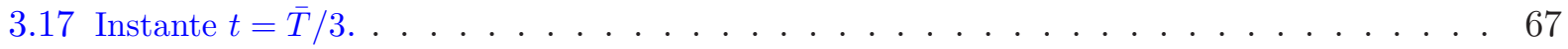

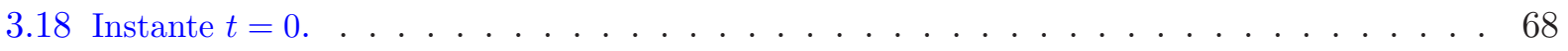

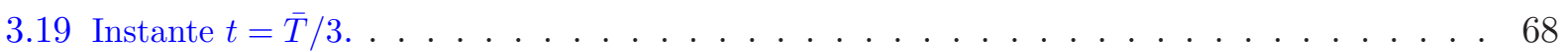

5.1 Dois exemplos de configurações centrais planares . . . . . . . . . . . . . . 85

5.2 Exemplo de Roberts: temos configurações centrais para todos os valores positivos de

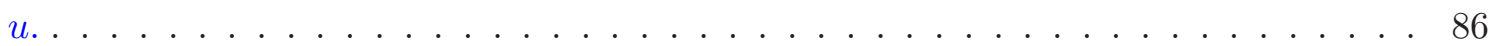

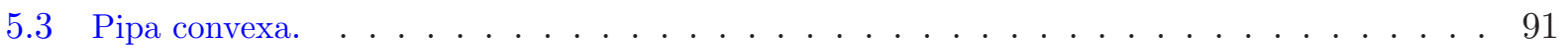

5.4 Pipa côncava. . . . . . . . . . . . . . . . . . . . . . . . 91

5.5 Configuração central do tipo Pipa. . . . . . . . . . . . . . . . . . 92

5.6 Regiões de existência de configurações centrais do tipo pipa. . . . . . . . . . . . . . . 96

5.7 Configurações centrais espaciais para 5-corpos. Configuração Côncava (a). Configuração Convexa (b) . . . . . . . . . . . . . . . . . . . . 97

5.8 Coordenadas para o problema com 5 corpos. . . . . . . . . . . . . . . . 98

5.9 Regiões abertas $\mathcal{H}_{1}, \mathcal{H}_{2}$ e $\mathcal{W} \ldots \ldots \ldots \ldots$

5.10 Região aberta U . . . . . . . . . . . . . . . . . . . 106

5.11 Os corpos com massas $m_{1}, m_{2}$ e $m_{3}$ estão nos vértices de um triângulo equilátero $\mathcal{T}$, o corpo com massa $m_{6}$ está no baricentro de $\mathcal{T}$ e os corpos com massas $m_{4}, m_{5}$ estão localizados

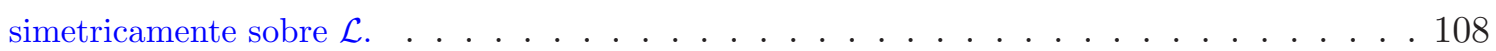

5.12 Solução homográfica para seis corpos. . . . . . . . . . . . . . . . . . 110 


\section{Capítulo 1}

\section{Introdução}

O movimento dos corpos celestes há muito tempo encanta os homens. Portanto, como funciona tal movimento foi uma das primeiras perguntas da dinâmica. Com o intuito de responder a essa pergunta diversos modelos foram criados. Dois destes seguem abaixo:

- Os gregos teocêntricos diziam que a terra ocupava o centro geométrico do universo e os corpos celestes moviam-se ao seu redor. Primeiro sugeriram que o movimento se dava sobre círculos concêntricos, o que em seguida se tornou insatisfatório, pois não explicava certas observações. Em 200 a.C. o astrônomo Ptolomeu de Alexandria propõe o uso de epiciclos, vide figura 1.1, mesmo assim alguns fenômenos ainda não eram explicados ou a sua descrição era muito complexa. Estas idéias foram aceitas até o século dezesseis;

- Na primeira parte do século XVI Nicolau Copérnico publica um trabalho intitulado De Revolutionibus Orbium Coelestium, vide [6], no qual faz a hipótese de que a terra e os demais planetas giravam ao redor do sol. Vide figura 1.2. Este modelo já havia sido sugerido 2000 anos antes pelo astrônomo grego Aristarco. Mas ambos não foram bem recebidos.

As idéias de Aristarco e Copérnico aproximam melhor o movimento dos corpos celestes, como é sabido hoje. Estes pensamentos serviram para os trabalhos de Galileu e de Kepler.

Os trabalhos de Galileu e Kepler dão início a estudos qualitativos da mecânica. Em seguida temos uma era quantitativa da mecânica que começa com Newton, quando ele resolve o problema de dois corpos no seu célebre [1]. Neste período quantitativo, no contexto da mecânica celeste um 


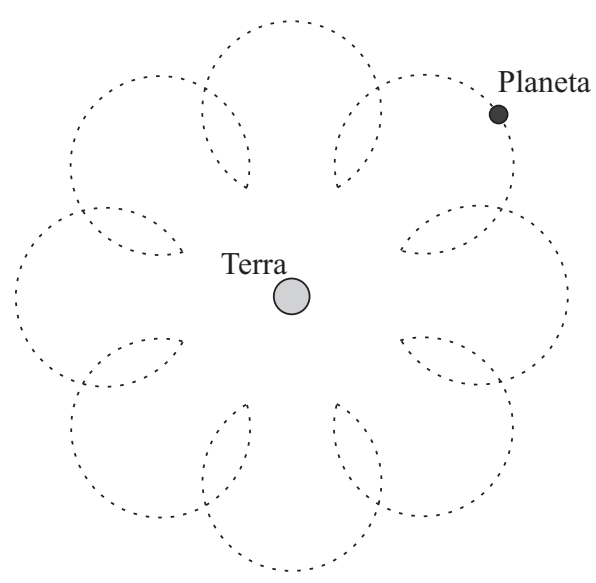

Figura 1.1: Representação da trajetória epicicloidal de um planeta no modelo de Ptolomeu.

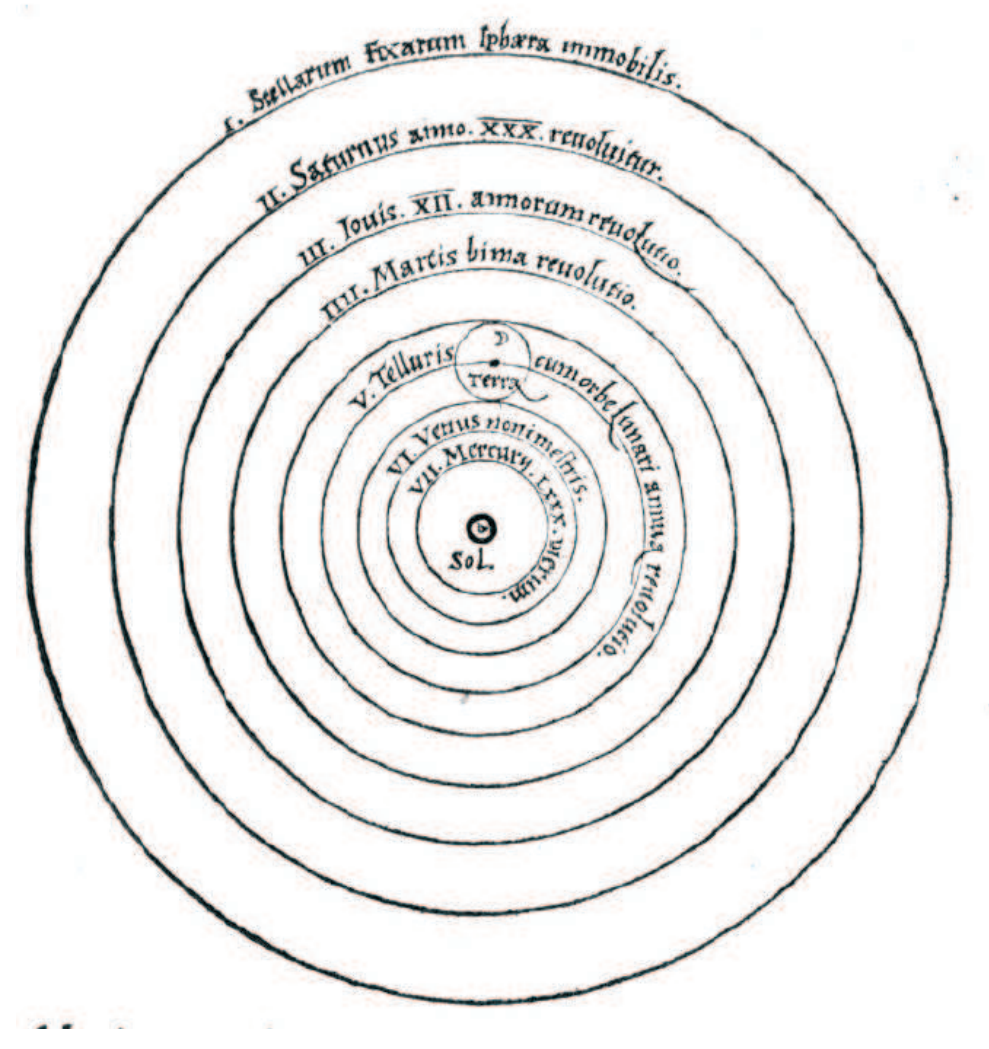

Figura 1.2: Representação do sistema solar segundo Copérnico [6].

problema bastante estudado foi o de $n$-corpos com forças gravitacionais, sobre o qual escreveram grandes nomes da ciência. Uma questão bastante abordada era a estabilidade do sistema solar, que foi analisada por Laplace usando expansão em série. Laplace concluiu que o sistema solar é 
estável, outros também o fizeram como Lagrange, Poisson, Dirichlet e Haretu, entre outros. Muitos resultados importantes foram obtidos neste período dentre eles as soluções de Euler e de Lagrange para o problema de três corpos, que serão mostradas a seguir. Recentemente uma nova solução para o problema de três corpos foi obtida por Chenciner e Montgomery, também será mostrada a seguir. Uma coleção de soluções homográficas também foi construída.

Em 1889 Poincaré mostrou que as séries usadas por Laplace et al divergiam para pequenas perturbações. Em 1887 Ernst Heinrich Bruns provou que nenhum método analítico envolvendo as equações algébricas usuais pode resolver o problema de $n$-corpos [2]. Estes dois eventos marcam o início da era qualitativa da mecânica. Após estas conclusões, Poincaré desenvolveu diversos métodos para fazer estudos qualitativos sobre as equações diferenciais, o que torna possível obter informações sobre as soluções de problemas, que como o de $n$-corpos, não tem solução explícita [3]. Muitos dos esforços para resolvê-lo quantitativamente não foram em vão. Nessas tentativas vários ramos da matemática e da mecânica se desenvolveram, tendo surgido técnicas de análise numérica, teoria dos sistemas dinâmicos, teoria de perturbações, métodos quantitativos e qualitativos das equações diferenciais, topologia, probabilidade, análise combinatória, geometrias diferencial e algébrica, entre outros [4].

Aqui abordaremos em especial o problema de Kepler, que na linguagem moderna é o problema de dois corpos com potencial Newtoniano. Mostraremos um teorema que relaciona a lei da gravitação de Newton e as leis de Kepler. Discorreremos também a cerca de alguns aspectos geométricos e topológicos do problema. Uma seção, em especial, é dedicada à regularização e à construção de equivalências entre o fluxo do problema de Kepler e o fluxo geodésico em superfícies de curvatura constante.

Por fim falaremos sobre o problema de $n$-corpos e suas soluções particulares conhecidas na literatura. Seguem algumas noções e notações que facilitarão o entendimento do texto. O leitor que estiver familiarizado com os formalismos da mecânica clássica pode omitir o restante desta seção. 


\subsection{Noções Preliminares da Mecânica Newtoniana}

Para complementação do texto introduzimos aqui algumas noções preliminares. Este assunto pode ser encontrado, por exemplo, em [5].

Definição 1.1.1 Chamaremos de partícula (ou corpo) todo ente físico cujas dimensões puderem ser esquecidas quando descrevemos seu movimento.

Definição 1.1.2 Os resultados obtidos levarão em conta os seguintes postulados de Newton:

1. Tempo(Absoluto) é uma noção exata e universal, e flui uniformemente sem relação com qualquer coisa externa;

2. Espaço(Absoluto) é uma noção exata e universal, e se estende uniformemente sem relação com qualquer coisa externa;

3. Referencial Inercial é uma coleção coerente de instrumentos de medida (réguas e relógios) capaz de determinar diferenças de Tempo Absoluto e de Espaço Absoluto;

4. Momento Linear é uma grandeza vetorial para cada sistema físico, que determina sua capacidade potencial de mudar sua vizinhança;

5. Força é uma grandeza vetorial que determina a maneira como os corpos sensíveis interagem mutuamente.

Estes enunciados podem ser encontrados em [7].

Definição 1.1.3 Num dado referencial inercial a posição de uma partícula é descrita por um vetor $\mathbf{r} \in \mathbb{R}^{3}$, então um sistema de $n$ partículas é descrito por $n$ vetores $\mathbf{r}_{1}, \ldots, \mathbf{r}_{n}$. A velocidade de uma partícula é a taxa de variação temporal de seu vetor posição,

$$
\mathbf{v}_{i}=\dot{\mathbf{r}}_{i}=\frac{d \mathbf{r}_{i}}{d t}
$$

Daqui por diante $\dot{x}$ denotará a derivada temporal de $x$. 
Definição 1.1.4 Chamaremos de massa da partícula a quantidade que relaciona seu momento linear com sua velocidade.

Com isto podemos enunciar as leis de Newton.

Definição 1.1.5 As leis que regem o movimento dos corpos segundo a mecânica Newtoniana são as seguintes:

1. Primeira Lei de Newton: Um sistema físico tem momento linear constante se sobre ele não atuam forças.

2. Segunda Lei de Newton: Seja $\mathrm{x}: \mathbb{R} \rightarrow \mathbb{R}^{3}$ a aplicação que descreve a trajetória de uma partícula de massa $m$ sob a ação de um campo vetorial

$$
\mathbf{F}: \mathbb{R}^{7} \rightarrow \mathbb{R}^{3}, \quad \mathbf{F}=\mathbf{F}(\mathbf{x}, \dot{\mathbf{x}}, t)
$$

Então,

$$
\frac{d}{d t}\{m \dot{\mathbf{x}}\}=\mathbf{F}(\mathbf{x}, \dot{\mathbf{x}}, t)
$$

3. Terceira Lei de Newton: Para cada força aplicada sobre um sistema físico corresponde uma reação com mesmo módulo e sentido oposto ao da força.

Definição 1.1.6 O momento angular de uma partícula de massa $m$ e vetor posição $\mathbf{r}$ é o seguinte vetor

$$
\mathbf{j}=\mathbf{r} \wedge m \dot{\mathbf{r}}
$$

Definição 1.1.7 Dado um sistema com $n$ massas $m_{1}, \ldots, m_{n}$ localizadas por $\mathbf{r}_{1}, \ldots, \mathbf{r}_{n}$, respectivamente, chamaremos de centro de massa o seguinte vetor

$$
\mathbf{X}=\frac{\sum_{i=1}^{n} m_{i} \mathbf{r}_{i}}{\sum_{i=1}^{n} m_{i}}
$$


Definição 1.1.8 Dado um sistema com $n$ massas $m_{1}, \ldots, m_{n}$ localizadas por $\mathbf{r}_{1}, \ldots, \mathbf{r}_{n}$, respectivamente. Chamaremos de momento linear total o seguinte vetor

$$
\mathbf{P}=\sum_{i=1}^{n} m_{i} \dot{\mathbf{r}}_{i} .
$$

Definição 1.1.9 Dado um sistema com $n$ massas $m_{1}, \ldots, m_{n}$ localizadas por $\mathbf{r}_{1}, \ldots, \mathbf{r}_{n}$, respectivamente. Chamaremos de momento angular total o seguinte vetor

$$
\mathbf{J}=\sum_{i=1}^{n} \mathbf{r}_{i} \wedge m_{i} \dot{\mathbf{r}}_{i}
$$

O porque destas quantidades serem tomadas com essas formas será dado logo a seguir.

Definição 1.1.10 Dado um sistema com $n$ massas $m_{1}, \ldots, m_{n}$ localizadas por $\mathbf{r}_{1}, \ldots, \mathbf{r}_{n}$, respectivamente. Chamaremos de momento de inercia do sistema a seguinte quantidade escalar

$$
I=\sum_{i=1}^{n} m_{i} \mathbf{r}_{i} \bullet \mathbf{r}_{i}
$$

Definição 1.1.11 Um campo de forças $\mathbf{F}: \mathbb{R}^{3 n} \rightarrow \mathbb{R}^{3}$ é dito conservativo se existe uma função escalar $V: \mathbb{R}^{3 n} \rightarrow \mathbb{R}$ tal que

$$
\mathbf{F}=-\sum_{i=1}^{n} \nabla_{i} V
$$

onde $\nabla_{i}$ é o gradiente em relação às coordenadas de $\mathbf{r}_{i}$. Chamamos $V$ de potencial do campo de forças $\mathbf{F}$.

Definição 1.1.12 Um campo de forças $\mathbf{F}: \mathbb{R}^{3 n} \rightarrow \mathbb{R}^{3}$ é dito central se existe uma função escalar $\phi: \mathbb{R}^{3 n} \rightarrow \mathbb{R}$ tal que

$$
\mathbf{F}_{i}=\phi\left(\mathbf{r}_{1}, \ldots, \mathbf{r}_{n}\right) \mathbf{r}_{i}
$$

onde $i=1, \ldots, n$.

Definição 1.1.13 Dado um sistema com $n$ massas $m_{1}, \ldots, m_{n}$ localizadas por $\mathbf{r}_{1}, \ldots, \mathbf{r}_{n}$, respec- 
tivamente. Chamaremos de energia cinética do sistema a seguinte quantidade escalar

$$
T=\frac{1}{2} \sum_{i=1}^{n} m_{i} \dot{\mathbf{r}}_{i} \bullet \dot{\mathbf{r}}_{i}
$$

Definição 1.1.14 Dado um sistema conservativo com $n$ massas $m_{1}, \ldots, m_{n}$ localizadas por $\mathbf{r}_{1}, \ldots, \mathbf{r}_{n}$, respectivamente. Chamaremos de energia mecânica do sistema a seguinte quantidade escalar

$$
E=T+V=\frac{1}{2} \sum_{i=1}^{n} m_{i} \dot{\mathbf{r}}_{i} \bullet \dot{\mathbf{r}}_{i}+V
$$

onde $V$ é o potencial é chamado de potencial do sistema.

Destas definições podemos retirar os seguintes resultados.

Teorema 1.1.15 Num sistema isolado com $n$ massas $m_{1}, \ldots, m_{n}$ localizadas por $\mathbf{r}_{1}, \ldots, \mathbf{r}_{n}$, respectivamente, o momento linear total do sistema se conserva.

Teorema 1.1.16 Num sistema conservativo com $n$ massas $m_{1}, \ldots, m_{n}$ localizadas por $\mathbf{r}_{1}, \ldots, \mathbf{r}_{n}$, respectivamente, a energia mecânica do sistema se conserva.

Teorema 1.1.17 Num sistema isolado com $n$ massas $m_{1}, \ldots, m_{n}$ localizadas por $\mathbf{r}_{1}, \ldots, \mathbf{r}_{n}$, respectivamente, se o campo de forças é central, o momento angular total do sistema se conserva.

\subsection{Noções Preliminares da Mecânica Lagrangiana}

Aqui mostramos algumas definições e resultados da mecânica Lagrangiana. As noções de tempo e espaço continuam as mesmas. Uma boa referência para este assunto é [8].

Definição 1.2.1 Entende-se por número de graus de liberdade de um sistema físico o número mínimo de quantidades necessárias para definir univocamente sua posição. Considerando um sistema com $\beta$ graus de liberdade, chamaremos de coordenadas generalizadas quaisquer $\beta$ quantidades $\left(q_{1}, \ldots, q_{\beta}\right) \equiv q \in \mathbb{R}^{\beta}$, que consigam definir univocamente sua posição. O conjunto de todas as coordenadas generalizadas é chamado de espaço de configurações. Em acordo com a definição 1.1.3 chamaremos $\left(\dot{q}_{1}, \ldots, \dot{q_{\beta}}\right) \equiv \dot{q} \in \mathbb{R}^{\beta}$ de velocidades generalizadas do sistema. 
Definição 1.2.2 Chamaremos de função principal de Lagrange ou Lagrangiana de um sistema físico a seguinte função escalar

$$
\begin{aligned}
L: \mathbb{R}^{\beta} \times \mathbb{R}^{\beta} \times \mathbb{R} & \rightarrow \mathbb{R} \\
& (q, \dot{q}, t) \mapsto L(q, \dot{q}, t)=T-V .
\end{aligned}
$$

Aqui $V$ e $T$ são as mesmas definidas em 1.1.11 e 1.1.13, porém devemos imaginar que elas foram escritas como acima e que, possivelmente, passaram por uma mudança de coordenadas que levou as antigas coordenadas cartesianas em novas coordenadas generalizadas.

A função principal de Lagrange contém todas as informações sobre o sistema físico. O conhecimento desta e o uso do princípio da mínima ação, que está descrito em 1.2.3, nos conduzirão a um sistema de equações diferenciais que nos permite determinar as equações de movimento. Em verdade, este sistema de equações diferenciais é equivalente aquele que obtemos usando a segunda lei de Newton.

Princípio 1.2.3 Suponha um sistema físico descrito, no espaço de configuração por $q(t)$, tal que $q\left(t_{1}\right)=q^{(1)}$ e $q\left(t_{2}\right)=q^{(2)}$, então o caminho que o sistema percorre de $q^{(1)}$ até $q^{(2)}$ é aquele $q(t)$ no qual o funcional

$$
\mathcal{S}=\int_{t_{1}}^{t_{2}} L(q, \dot{q}, t) d t
$$

é extremizado. O funcional $\mathcal{S}$ é chamado de ação do sistema.

De modo análogo podemos escrever o princípio da mínima ação como uma equação variacional,

$$
\delta \mathcal{S}=\delta \int_{t_{1}}^{t_{2}} L(q, \dot{q}, t) d t=0
$$

que é dizer que o caminho $q(t)$ que leva de $q^{(1)}$ até $q^{(2)}$ é aquele no qual a primeira variação de $\mathcal{S}$ é nula, mantendo $q\left(t_{1}\right)=q^{(1)}$ e $q\left(t_{2}\right)=q^{(2)}$ fixos.

Vamos desenvolver 1.1, notando que a variação no tempo é nula. Daí temos,

$$
\delta \mathcal{S}=\int_{t_{1}}^{t_{2}}\left[\frac{\partial L}{\partial q} \bullet \delta q+\frac{\partial L}{\partial \dot{q}} \bullet \delta \dot{q}\right] d t=0
$$


ou ainda,

$$
\delta \mathcal{S}=\int_{t_{1}}^{t_{2}}\left[\frac{\partial L}{\partial q} \bullet \delta q+\frac{d}{d t}\left(\frac{\partial L}{\partial \dot{q}} \bullet \delta q\right)-\frac{d}{d t}\left(\frac{\partial L}{\partial \dot{q}}\right) \bullet \delta q\right] d t=0 .
$$

Integrando o segundo termo temos

$$
\delta \mathcal{S}=\left[\frac{\partial L}{\partial \dot{q}} \bullet \delta q\right]_{t_{1}}^{t_{2}}+\int_{t_{1}}^{t_{2}}\left[\frac{\partial L}{\partial q}-\frac{d}{d t}\left(\frac{\partial L}{\partial \dot{q}}\right)\right] \bullet \delta q d t=0
$$

como os extremos estão fixos o primeiro termo se anula, devemos ter então

$$
\frac{\partial L}{\partial q}-\frac{d}{d t}\left(\frac{\partial L}{\partial \dot{q}}\right)=0
$$

Definição 1.2.4 As $\beta$ equações diferenciais em 1.2 são chamadas de equações de Euler-Lagrange.

Juntas as equações em 1.2 determinam completamente a dinâmica do sistema a menos das $2 \beta$ constantes de integração, analogamente à segunda lei de Newton.

Decorre imediatamente o seguinte resultado

Teorema 1.2.5 Suponha que a Lagrangiana de um sistema seja $L=L(q, \dot{q}, t)$, então a adição de qualquer função escalar $g(q, t)$ que seja a derivada total, com respeito ao tempo, de outra função não altera as equações de movimento do sistema.

Podemos usar algumas propriedades da função principal de Lagrange para mostrar leis de conservação semelhantes àquelas mostradas nos três primeiros teoremas.

Definição 1.2.6 Chamaremos de momento canonicamente conjugado o seguinte vetor

$$
p_{k}=\frac{\partial L}{\partial q_{k}}
$$

Note que no caso de coordenadas cartesianas este coincide com o momento linear dos postulados de Newton. 
Teorema 1.2.7 Suponha que a Lagrangiana de um sistema físico seja dada por

$$
L=L\left(q_{1}, \ldots, q_{k-1}, q_{k+1}, \ldots, q_{\beta}, \dot{q}_{1}, \ldots, \dot{q}_{\beta}, t\right),
$$

isto é, a Lagrangiana não é função da k-ésima coordenada generalizada. Então o k-ésimo momento conjugado é uma constante do movimento.

Quando a Lagrangiana não é função da $k$-ésima coordenada generalizada dizemos que esta coordenada é cíclica.

Teorema 1.2.8 Suponha que a Lagrangiana de um sistema isolado seja L. Pelos postulados de Newton o tempo flui uniformemente e o espaço é homogêneo e isotrópico, ou seja, a Lagrangiana não depende explicitamente do tempo e o potencial depende apenas das coordenadas. Então associado a estes fatos temos as seguintes leis de conservação.

1. A homogeneidade do tempo implica na conservação da energia;

2. A homogeneidade do espaço implica na conservação do momento linear total do sistema;

3. A isotropia do espaço implica na conservação do momento angular total do sistema.

Por fim vamos definir um sistema de coordenadas que por vezes é útil.

Definição 1.2.9 Considerando um sistema formado por $n$-massas, localizadas por $q_{1}, \ldots, q_{n}$, respectivamente. Definimos as coordenadas de Jacobi como segue

$$
\left\{\begin{array}{l}
\xi_{0}=\sum_{i=1}^{n} m_{i} q_{i} / \sum_{i=1}^{n} m_{i} \\
\xi_{1}=q_{1}-q_{2} \\
\xi_{2}=q_{3}-\sum_{i=1}^{2} m_{i} q_{i} / \sum_{i=1}^{2} m_{i} \\
\vdots \\
\xi_{n}=q_{n}-\sum_{i=1}^{n-1} m_{i} q_{i} / \sum_{i=1}^{n-1} m_{i}
\end{array}\right.
$$

Passemos agora ao problema de Kepler onde usaremos algumas destas notações. 


\section{Capítulo 2}

\section{O Problema de Kepler}

Neste capítulo vamos tratar o problema de dois corpos interagindo gravitacionalmente.

Em um primeiro momento trabalhamos com o caso geral. Nas seções seguintes, levando em conta a conservação dos momentos linear total e angular total, consideramos diretamente o problema reduzido. Dessa forma, após uma escolha conveniente de unidades temos de estudar a seguinte equação diferencial

$$
\frac{d^{2} q}{d t^{2}}=-\frac{q}{|q|^{3}},
$$

onde $|\cdot|$ denota a norma Euclidiana usual.

A Hamiltoniana neste caso se escreve como

$$
H=\frac{|p|^{2}}{2}-\frac{1}{|q|} .
$$

Na seção 2.2 apresentamos alguns fatos geométricos acerca das órbitas, no espaço de configuração e no espaço de fase, decorrentes das simetrias desta função Hamiltoniana.

Mostramos a equivalência entre o problema de Kepler e o fluxo geodésico em superfícies de curvatura constante. 


\subsection{Leis de Kepler e a Gravitação de Newton}

Entre 1609 e 1619 o matemático, astrônomo e astrólogo Johannes Kepler publicou, entre outros trabalhos, Astronomia Nova [10] e Harmonice Mundi [11]. Baseado nas idéias heliocêntricas de Nicolau Copérnico e nos dados de mais de 14 anos de observação do observatório de Tycho Brahe, Kepler postulou três leis, hoje chamadas leis de Kepler, as quais seguem

1. Primeira Lei: Os Planetas se movem em órbitas elípticas com o Sol num dos focos, [10];

2. Segunda Lei: O segmento que vai do Sol ao Planeta varre áreas iguais em tempos iguais, [10];

3. Terceira Lei: O período de revolução da órbita é proporcional à potência três meios do eixo maior da elipse, [11].

Em seguida, vem o problema de dizer se estas leis podem ser, ou não, obtidas a partir de noções fundamentais. Para isto iniciam-se discussões sobre a dinâmica do movimento planetário e, por conseguinte, tentativas de descrição da lei de interação que regia tal movimento. Com este propósito Isaac Newton formula em 1666 a lei da gravitação universal e a publica em 1687 no seu Philosophiae Naturalis Principia Mathematica [1].

Lei da Gravitação Universal: A interação gravitacional entre dois corpos pode ser expressa por uma força central, atrativa, proporcional ao produto das massas destes corpos e inversamente proporcional ao quadrado da distância entre eles [5].

Assim, em módulo, a força que um exerce no outro é dada por

$$
F=\frac{G m_{1} m_{2}}{d^{2}}
$$

onde $m_{1}$ e $m_{2}$ representam as massas dos corpos, $d$ a distância entre eles e $G$ é uma constante de proporcionalidade, que de acordo com medições recentes [12] é dada por

$$
G=(6,67259 \pm 0,00085) \cdot 10^{-11} N \cdot m^{2} \cdot K g^{-2} .
$$


Vamos considerar o problema no contexto abordado por Newton e Kepler, que é interação dos Planetas com o Sol. Assim vamos enunciar e provar o seguinte

Teorema 2.1.1 Considere um sistema formado pelo Sol e um outro planeta qualquer e que a interação entre eles seja independente da velocidade. Então o movimento deste planeta é dado pelas leis de Kepler se, e somente se, a interação é dada na forma da lei da gravitação universal de Newton.

Prova: Mostraremos primeiro a recíproca, isto é, a lei da gravitação universal implica nas leis de Kepler.

Suponha que seja válida a lei da gravitação universal. Então, considerando como partículas o Sol, com massa $m_{1}$, e o planeta, com massa $m_{2}$, com posições dadas por $q_{1}, q_{2} \in \mathbb{R}^{3}$, respectivamente. Não vamos considerar o caso da colisão. Assim temos a seguinte Lagrangeana para o sistema

$$
L:\left(\mathbb{R}^{6}-\{0\}\right) \times\left(\mathbb{R}^{6}\right) \rightarrow \mathbb{R}
$$

onde

$$
L\left(q_{1}, q_{2}, \dot{q}_{1}, \dot{q_{2}}\right)=\frac{1}{2}\left(m_{1}{\dot{q_{1}}}^{2}+m_{2}{\dot{q_{2}}}^{2}\right)+\frac{G m_{1} m_{2}}{\left|q_{1}-q_{2}\right|} .
$$

Mudando para coordenadas de Jacobi ela se escreve como

$$
L\left(\xi_{0}, \xi_{1}, \dot{\xi_{0}}, \dot{\xi_{1}}\right)=\left(\frac{m_{1}+m_{2}}{2}\right){\dot{\xi_{0}}}^{2}+\frac{m_{1} m_{2}}{2\left(m_{1}+m_{2}\right)}{\dot{\xi_{1}}}^{2}+\frac{G m_{1} m_{2}}{\left|\xi_{1}\right|} .
$$

A Lagrangeana em 2.3 conduz a uma equação diferencial em $\mathbb{R}^{12}$. Para cada corpo devemos determinar uma 6-upla, na qual três coordenadas são posições e as outras velocidades. Como $\xi_{0}$ é cíclica temos

$$
\xi_{0}(t)=\xi_{0}(0)+t \dot{\xi}_{0}(0)
$$

donde precisamos estudar apenas a Lagrangeana

$$
L=\frac{m_{1} m_{2}}{2\left(m_{1}+m_{2}\right)} \dot{\xi}_{1}^{2}+\frac{G m_{1} m_{2}}{\left|\xi_{1}\right|} .
$$


Vemos que 2.4 conduz a uma equação diferencial em $\mathbb{R}^{6}$ da seguinte forma

$$
\frac{m_{1} m_{2}}{m_{1}+m_{2}} \ddot{\xi}_{1}+G m_{1} m_{2} \frac{\xi_{1}}{\left|\xi_{1}\right|^{3}}=0
$$

Como estamos falando do Sol interagindo com um outro planeta temos $m_{1}>>m_{2}$, o que para o caso Sol e Terra por exemplo é bastante razoável, uma vez que estima-se que no sistema solar a soma das massas de todos os planetas e asteróides corresponda a 0,2\% da massa do Sol, vide [13], daí podemos fazer

$$
\ddot{\xi_{1}}+G m_{1} \frac{\xi_{1}}{\left|\xi_{1}\right|^{3}}=0
$$

Com isto estaremos impondo que o centro de massa do sistema, praticamente, coincida com o do Sol.

Conforme mencionado no capítulo 1, para estes problemas consideramos o espaço como sendo isotrópico, portanto a lagrangeana 2.4 é invariante por rotações rígidas. Este fato nos leva à conservação do vetor momento angular $\mathbf{J}$ do sistema. Assim o movimento da órbita da equação 2.5 se dá num plano fixo, ortogonal ao vetor momento angular. Vamos usar coordenadas polares sobre este plano, conforme indicado na figura 2.1.

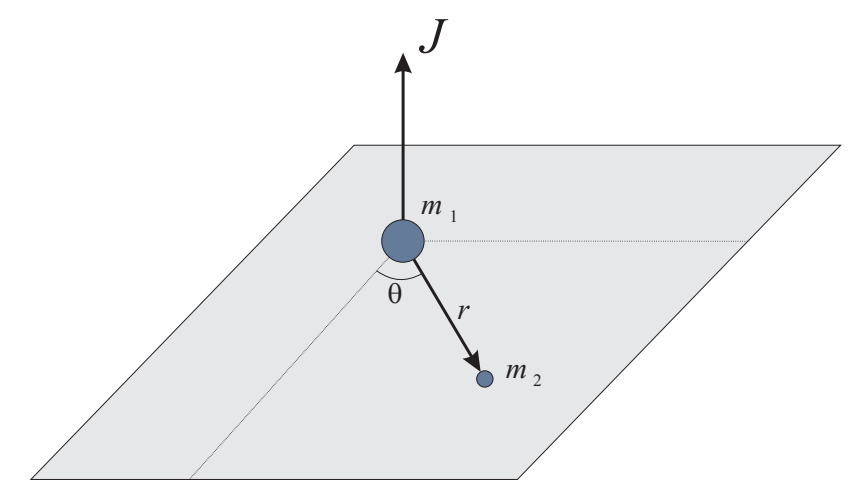

Figura 2.1: Plano de movimento onde evidenciamos o uso de coordenadas polares. 
Com a observação da conservação do momento angular vemos que 2.5 é na verdade uma equação diferencial em $\mathbb{R}^{4}$. Usando coordenadas polares para a posição temos,

$$
\xi_{1}=(r \cos \theta, r \sin \theta)
$$

O módulo do momento angular, que é conservado, em coordenadas polares fica dado por

$$
J=m_{2} r^{2} \dot{\theta}
$$

enquanto que a energia cinética fica dada por

$$
T=\frac{m_{2}}{2}\left(\dot{r}^{2}+r^{2} \dot{\theta}^{2}\right)
$$

Juntando 2.6 e 2.7 à definição da energia mecânica do sistema, que também se conserva visto que a Lagrangeana 2.3 não tem dependência explícita no tempo, temos

$$
E=\frac{m_{2} \dot{r}^{2}}{2}+\frac{J^{2}}{2 m_{2} r^{2}}-\frac{G m_{1} m_{2}}{r}
$$

onde usamos

$$
\dot{\theta}=\frac{J}{m_{2} r^{2}}=\frac{d \theta}{d t}
$$

Voltemos nossa atenção à equação 2.6, nesta vemos que $\dot{\theta}$ varia com $|r|$ e não muda de sinal durante o movimento, pois é razoável supor que $J \neq 0$, assim pelo teorema da função implícita podemos fazer $\theta=\theta(r)$. Desta feita podemos usar 2.8 e 2.9 para fazer

$$
\dot{r}=\frac{d r}{d t}=\sqrt{\frac{2}{m_{2}}\left(E-\frac{J^{2}}{2 m_{2} r^{2}}+\frac{G m_{1} m_{2}}{r}\right)}=\frac{d r}{d \theta} \frac{J}{m_{2} r^{2}},
$$

donde

$$
d \theta=\frac{J d r}{r^{2} \sqrt{2 m_{2} E-\frac{J^{2}}{r^{2}}+\frac{2 k}{r}}}
$$


onde $k=G m_{1} m_{2}{ }^{2}$. Integrando esta expressão obtemos a menos de uma constante aditiva

$$
\theta(r)=\arccos \left(\frac{J / r-k / J}{\sqrt{2 m_{2} E+\frac{k^{2}}{J^{2}}}}\right) .
$$

Invertendo esta relação obtemos

$$
r(\theta)=\frac{\rho}{1+\varepsilon \cos \theta}
$$

com

$$
\rho=\frac{J^{2}}{k} \quad, \quad \varepsilon=\sqrt{2 m_{2} E+\frac{k^{2}}{J^{2}}} .
$$

A expressão 2.12 representa a equação de uma elipse em coordenadas polares, vide figura 2.3, desde que $0<\varepsilon<1$. Isto mostra parcialmente a validade da primeira lei de Kepler. Para ver que a condição $0<\varepsilon<1$ é verificada podemos analisar como $\varepsilon$ varia com a energia. Para isto devemos analisar os gráficos do chamado potencial efetivo,

$$
V_{e f}=\frac{J^{2}}{2 m_{2} r^{2}}-\frac{G m_{1} m_{2}}{r}
$$

Vide figura 2.2 abaixo

Temos que observar empiricamente que no caso de interesse o movimento se dá numa região confinada, isto é com energia negativa. Para valores positivos de energia, $(\varepsilon>1)$, temos ramos de hipérboles e para o valor nulo, $(\varepsilon=1)$, temos uma parábola, ambas são soluções ilimitadas. Portanto, não representam as observações.

Passemos agora ao segundo postulado de Kepler e mostremos que este decorre diretamente da conservação da norma do momento angular. Para a trajetória elíptica descrita acima teremos a seguinte equação para o cálculo do elemento de área, $\mathcal{A}$, varrida pelo vetor posição

$$
d \mathcal{A}=\frac{r^{2} d \theta}{2}=\frac{r^{2}}{2} \frac{d \theta}{d t} d t
$$




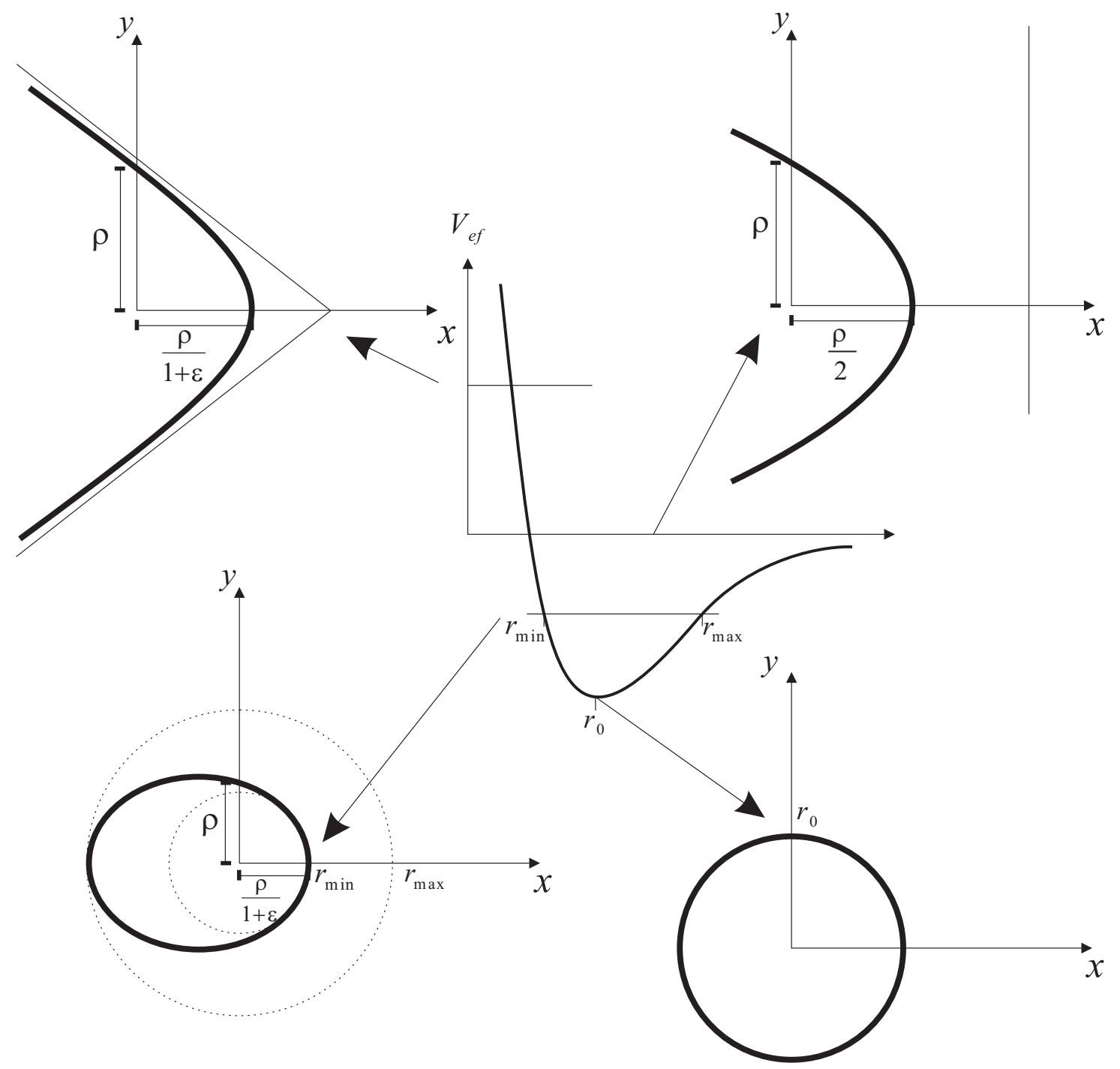

Figura 2.2: Possíveis movimentos para $J \neq 0$.

ou usando 2.6,

$$
d \mathcal{A}=\frac{J}{2} d t
$$

Como $J$ é conservada a área $\mathcal{A}$ é função linear do tempo e isto implica que para tempos iguais teremos áreas iguais. Disto decorre a segunda lei de Kepler, que é conhecida como lei das áreas, vide figura 2.4 .

Observação 2.1.2 Um fato interessante a se mencionar nesse ponto é a interpretação de Kepler 


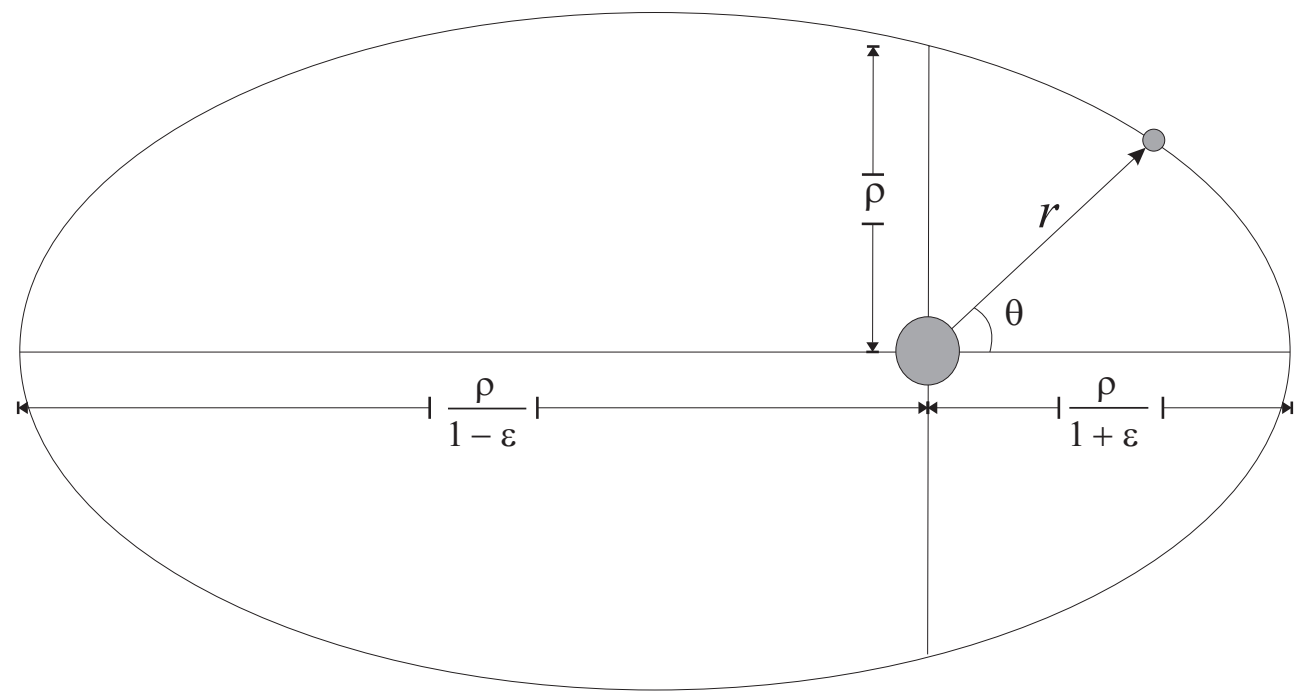

Figura 2.3: Órbita em forma de elipse.

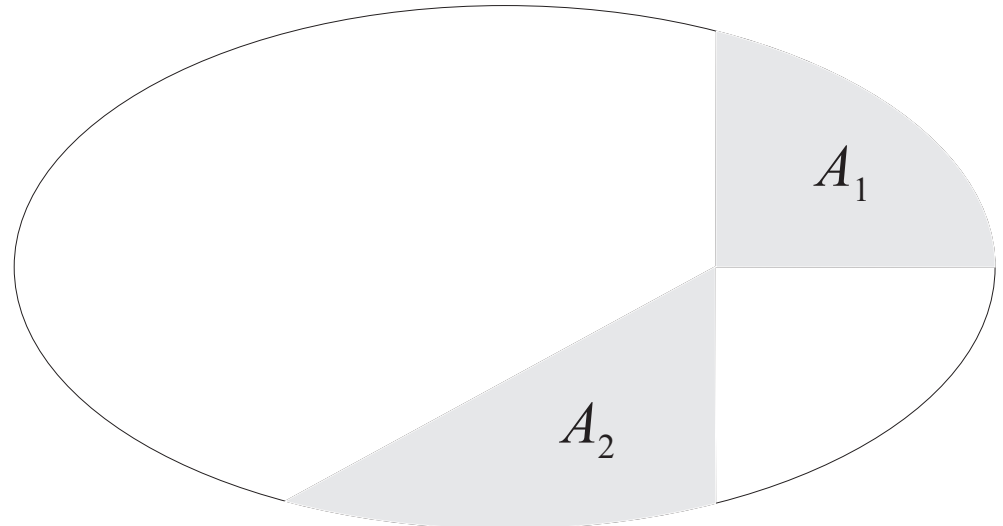

Figura 2.4: Lei das áreas $A_{1}=A_{2}$.

acerca dessa lei, [10]. Uma vez que ele não conhecia o conceito de inércia ele acreditava que o Sol impelia uma força lateral inversamente proporcional à distância sobre os Planetas, (entenda-se por força lateral a componente tangencial que estaria na direção do vetor velocidade). Bastante diferente da interpretação atual.

Para a terceira lei de Kepler consideremos a equação 2.12 e passemo-la para coordenadas carte$\operatorname{sianas}(r \cos \theta, r \sin \theta)=(x, y)$. Com tal procedimento teremos

$$
\frac{\left(1-\varepsilon^{2}\right)^{2}}{\rho^{2}}\left(\frac{\rho \varepsilon}{1-\varepsilon^{2}}+x\right)^{2}+\left(\frac{1-\varepsilon^{2}}{\rho^{2}}\right) y^{2}=1
$$


Se observamos ainda a forma padrão para a equação da elipse em coordenadas cartesianas, a qual seja

$$
\frac{(a \varepsilon+x)^{2}}{a^{2}}+\frac{y^{2}}{b^{2}}=1
$$

Fazendo a correspondência desta com a 2.14, vemos que

$$
a=\frac{\rho}{1-\varepsilon^{2}}
$$

e

$$
b=\frac{\rho}{\sqrt{1-\varepsilon^{2}}} .
$$

Sendo que $a$ é o semi-eixo maior da elipse e $b$ o semi-eixo menor. Sabendo que a área total da elipse é dada por

$$
\mathcal{A}=\pi a b
$$

e considerando a expressão 2.13 , temos

$$
\mathcal{A}(t)=\frac{J}{2} t
$$

de tal forma que a área total será

$$
\mathcal{A}=\frac{J}{2} \tau
$$

onde $\tau$ representa o período de revolução da órbita elíptica.

Tomando os resultados de 2.15 e 2.16 temos

$$
2 \pi a b=J \tau
$$

inserindo nesta as expressões para os semi-eixos nos vem

$$
\tau=\frac{2 \pi}{J} \frac{\rho}{\left(1-\varepsilon^{2}\right)} \frac{\rho}{\sqrt{1-\varepsilon^{2}}}=\frac{2 \pi \rho^{1 / 2}}{J} \frac{\rho^{3 / 2}}{\left(1-\varepsilon^{2}\right)^{3 / 2}} .
$$


Portanto, com uma constante

$$
C=\frac{2 \pi}{\sqrt{k}}
$$

temos

$$
\tau=C a^{3 / 2}
$$

que é a terceira lei de Kepler.

Observação 2.1.3 Para chegarmos à expressão 2.12 fizemos uso de um método, que consistia em buscar as integrais primeiras do problema e usá-las para reduzir os graus de liberdade do sistema. Assim um sistema inicialmente em $\mathbb{R}^{12}$, foi posto em $\mathbb{R}^{6}$ usando as seis quantidades conservadas do momento linear. Em seguida, usando a conservação do módulo, da direção e do sentido do momento angular total levou-se o sistema para $\mathbb{R}^{3}$. Por fim usamos a conservação da energia total do sistema para obter uma equação em $\mathbb{R}^{2}$ e resolver para $r$ em função de $\theta$. Em verdade, usou-se o fato de existirem 10 integrais primeiras no problema de dois corpos para reduzir o número de graus de liberdade do sistema de doze para dois.

Vamos mostrar agora que as leis de Kepler implicam na lei da gravitação universal de Newton.

Considere que sejam válidas as leis de Kepler para o movimento planetário, assim por hipótese temos que o movimento é planar. Por conseguinte, podemos usar coordenadas polares para descrever tal movimento. Tomando a expressão da aceleração em coordenadas polares obtemos para um planeta de massa $m_{p}$ as seguintes expressões

$$
\left\{\begin{array}{l}
m_{p}\left(\ddot{r}-r \dot{\theta}^{2}\right)=F \\
2 \dot{r} \dot{\theta}+r \ddot{\theta}=0
\end{array} .\right.
$$

A segunda equação de 2.17 é equivalente à conservação do momento angular, podemos multiplicar por $r$ em ambos os lados e obter

$$
2 r \dot{r} \dot{\theta}+r^{2} \ddot{\theta}=\frac{d}{d t}\left(r^{2} \dot{\theta}\right)=0
$$


daí

$$
m_{p} r^{2} \dot{\theta}=J=\text { constante. }
$$

Como podemos ver a função $\theta$ não troca de sinal durante o movimento, isso implica que pelo teorema da função implícita podemos encontrar $r=r(\theta)$. Usando a regra da cadeia para derivação temos

$$
\frac{d}{d \theta}\left(\frac{1}{r}\right)=-\frac{\dot{r}}{J}
$$

e ainda

$$
\frac{d^{2}}{d \theta^{2}}\left(\frac{1}{r}\right)=-\frac{\ddot{r}}{\dot{\theta} J} .
$$

Juntando 2.19 com a primeira equação de 2.17 temos

$$
\frac{d^{2}}{d \theta^{2}}\left(\frac{1}{r}\right)+\left(\frac{1}{r}\right)=-\frac{F r^{2}}{m_{p} J^{2}}
$$

Esta expressão é conhecida como fórmula de Binet, e serve para determinar a órbita se conhecemos a interação ou a interação se conhecemos a órbita. Aqui usaremos seu segundo propósito:

$$
F=-\frac{m_{p} J^{2}}{r^{2}}\left[\frac{d^{2}}{d \theta^{2}}\left(\frac{1}{r}\right)+\left(\frac{1}{r}\right)\right]
$$

Partimos agora da expressão de uma elipse em coordenadas polares para a órbita, pois assumimos a priori a primeira lei de Kepler. Donde

$$
\frac{1}{r}=\frac{1+\varepsilon \cos \theta}{\rho}
$$

Usando esta última em 2.20 temos,

$$
F=-\frac{m_{p} J^{2}}{r^{2}}\left[-\frac{\varepsilon \cos \theta}{\rho}+\frac{1+\varepsilon \cos \theta}{\rho}\right]
$$


o que nos dá

$$
F=-\frac{m_{p} J^{2}}{\rho} \frac{1}{r^{2}}
$$

Para que a expressão 2.21 nos dê a lei da gravitação resta mostrar que $J^{2} / \rho$ é constante para todos os planetas. Para isto considere a segunda lei de Kepler, isto é, tome a expressão 2.18 para calcular a área da elipse. Assim nos vem

$$
\frac{d \mathcal{A}}{d t}=\frac{J}{2}
$$

Com esta expressão podemos obter o período de revolução da órbita fazendo

$$
\tau=\frac{\mathcal{A}}{d \mathcal{A} / d t}=\frac{2 \pi c_{1} c_{2}}{J}
$$

onde $c_{1}$ e $c_{2}$ são, respectivamente, o semi-eixo maior e o semi-eixo menor da elipse. Nas relações da elipse é fácil ver que $\rho=c_{2}^{2} / c_{1}$. Tomando o quadrado de 2.22 e dividindo por $c_{1}^{3}$, temos a seguinte expressão

$$
\frac{\tau^{2}}{c_{1}{ }^{3}}=\frac{4 \pi^{2} c_{2}^{2}}{c_{1} J^{2}}=\frac{4 \pi^{2} \rho}{J^{2}} .
$$

Finalmente considerando terceira lei de Kepler, vemos que o lado direito da expressão 2.23 deve ser uma constante para todos os planetas. Desta feita temos que 2.21 é equivalente à lei da gravitação universal de Newton [14].

\subsection{Alguns Aspectos Geométricos do Problema de Kepler}

Em caráter de complementação colocaremos a seguir um resultado de Willian Rowan Hamilton. Para isto vamos considerar diretamente a expressão

$$
\frac{d^{2} \mathbf{x}}{d t^{2}}=-\frac{k \mathbf{x}}{|\mathbf{x}|^{3}}
$$

onde $\mathbf{x} \in \mathbb{R}^{3}$ e $k$ é um número real positivo. Esta equação é chamada de equação de Kepler. Como vimos acima o problema de dois corpos pode ser reduzido à forma 2.24. 
Para o teorema a seguir assumiremos a equação $2.24 \mathrm{com}$ condições iniciais em $t=t_{0}$

$$
\mathbf{x}\left(t_{0}\right)=\mathbf{x}_{0}
$$

$\mathrm{e}$

$$
\mathbf{v}\left(t_{0}\right)=\frac{d \mathbf{x}}{d t}\left(t_{0}\right)=\mathbf{v}_{0}
$$

mas de forma que $\mathbf{x}_{0}$ e $\mathbf{v}_{0}$ sejam linearmente independentes. Segue o resultado de Hamilton de 1846, vide [15], um enunciado em linguagem mais simples pode ser encontrado em [16].

Teorema 2.2.1 Quando t varia o vetor velocidade se move ao longo de um círculo $\mathcal{C}$, o qual está no mesmo plano $\mathcal{P}$ que contém a origem, mas em geral não está centrado nela. Qualquer círculo pode ocorrer. Este círculo juntamente com a orientação determinam a órbita univocamente.

Prova: Como mencionado na prova do teorema anterior temos as seguintes integrais primeiras para 2.24

$$
\mathbf{J}=\mathbf{x} \times \mathbf{v}
$$

$\mathrm{e}$

$$
E=\frac{|\mathbf{v}|^{2}}{2}-\frac{k}{|\mathbf{x}|}
$$

2.25 nos dá que o movimento é planar, vamos então escolher um referencial no qual o vetor posição se escreva, em coordenadas polares, como

$$
\mathbf{x}=(r \cos \theta, r \sin \theta, 0)
$$

Desta forma, como antes, o módulo do momento angular é dado por

$$
J=r^{2} \frac{d \theta}{d t}
$$


Vamos escrever a equação de Newton em coordenadas polares também, donde

$$
\frac{d \mathbf{v}}{d t}=-\frac{k}{r^{2}}(\cos \theta, \sin \theta, 0)
$$

Como no teorema anterior, vamos usar o argumento da não mudança de sinal de $d \theta / d t$ com $r$ durante o movimento e usar o teorema da função implícita para fazer $\theta=\theta(r)$. Em seguida aplicando a regra da cadeia à equação 2.27 , temos

$$
\frac{d \mathbf{v}}{d \theta}=-R(\cos \theta, \sin \theta, 0)
$$

onde $R=k / J$. Integrando 2.28 temos

$$
\mathbf{v}=R(-\sin \theta, \cos \theta, 0)+\mathbf{c}
$$

onde $\mathbf{c}=\left(c_{1}, c_{2}, 0\right)$ é uma constante de integração. Isto nos mostra que o vetor velocidade se move ao longo de um círculo de raio $R=k / J$, centrado em c e está no plano ortogonal ao momento angular.

É interessante notarmos que $\mathbf{v}$ - $\mathbf{c}$ é sempre ortogonal a $\mathbf{x}$. Daí medir o ângulo entre dois vetores velocidade sobre o círculo $\mathcal{C}$ é o mesmo que medir o ângulo os respectivos vetores posição na origem.

A razão $\varepsilon=|\mathbf{c}| / R$ é chamada excentricidade de $\mathcal{C}$ e podemos escolher coordenadas de forma que

$$
\mathbf{v}=R(-\sin \theta, \varepsilon+\cos \theta, 0)
$$

Desta feita,o módulo do momento angular fica dado por,

$$
J=r R(1+\varepsilon \cos \theta)
$$

resolvendo para $r$ temos, novamente,

$$
r=\frac{\rho}{1+\varepsilon \cos \theta}
$$


$\operatorname{com} \rho=J^{2} / k$.

Usando a equação de Binet como acima veremos que esta expressão para $r$ satisfaz à equação de Newton.

Vale ressaltar que no caso do movimento hiperbólico a origem está fora do círculo de velocidades, no caso parabólico a origem está exatamente sobre o círculo de velocidades e, por fim, no caso elíptico a origem se encontra dentro do círculo de velocidades. A seguir, colocamos as figuras que mostram os três tipos possíveis de movimento, dentro da hipótese de independência linear das condições iniciais que fizemos acima.

No caso do movimento elíptico, como pode ser visto na figura 2.5, o círculo de velocidades é completamente percorrido, assim podemos falar de duas velocidades sobre uma mesma reta que passa pela origem. Temos o seguinte

Lema 2.2.2 Sejam $V_{1}$ e $\boldsymbol{V}_{2}$ duas velocidades sobre o círculo $\mathcal{C}$, as quais estão sobre uma linha que passa pela origem. Então,

$$
V_{1} \cdot V_{2}=c \cdot c-R^{2}=2 E
$$

Prova: Considere $\mathbf{w}$ diametralmente oposto a $\mathbf{V}_{1}$, assim existe e tal que,

$$
\mathbf{V}_{1}=\mathbf{c}+\mathbf{e}
$$

e

$$
\mathbf{w}=\mathbf{c}-\mathbf{e}
$$

donde vemos que

$$
\mathbf{w} \cdot \mathbf{V}_{1}=\mathbf{c} \cdot \mathbf{c}-\mathbf{e} \cdot \mathbf{e}=\mathbf{c} \cdot \mathbf{c}-R^{2}=2 E .
$$

Como $\mathbf{V}_{1}, \mathbf{V}_{2}$ e $\mathbf{w}$ formam um triângulo retângulo, segue que $\mathbf{V}_{1}$ é ortogonal a $\mathbf{V}_{2}-\mathbf{w}$. Daí $\mathbf{V}_{1} \cdot \mathbf{V}_{2}=\mathbf{V}_{1} \cdot \mathbf{w}$ e temos o resultado do lema. 


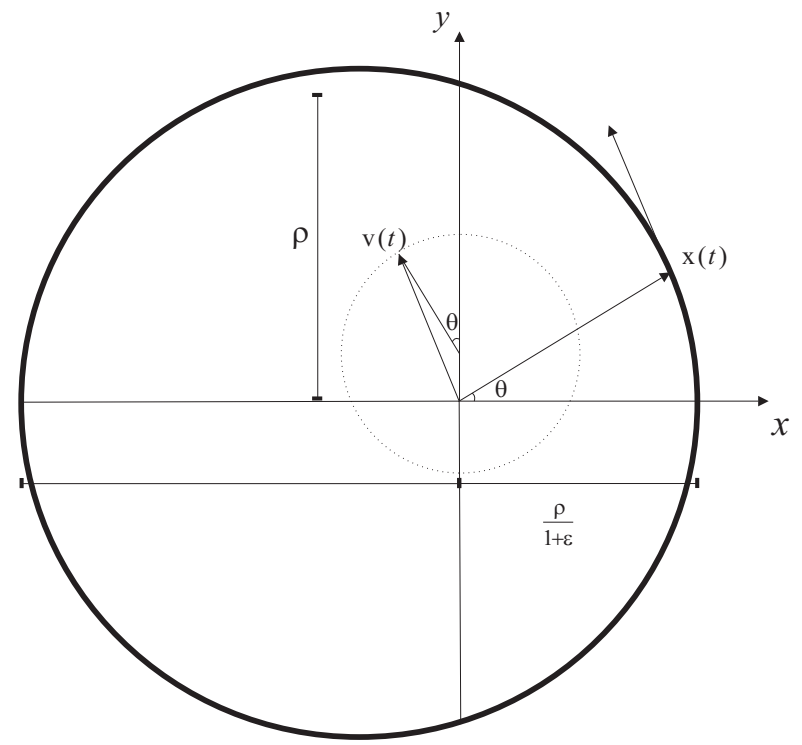

Figura 2.5: Hodógrafo do caso $E<0$, note que o círculo de velocidades é completamente percorrido.

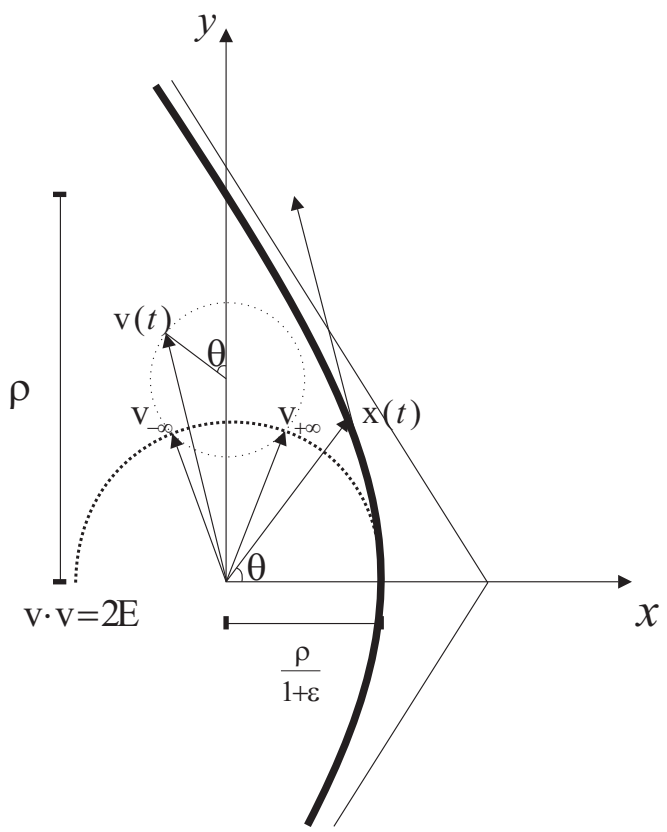

Figura 2.6: Hodógrafo do caso $E>0$, note que o círculo de velocidades não é completamente percorrido. 


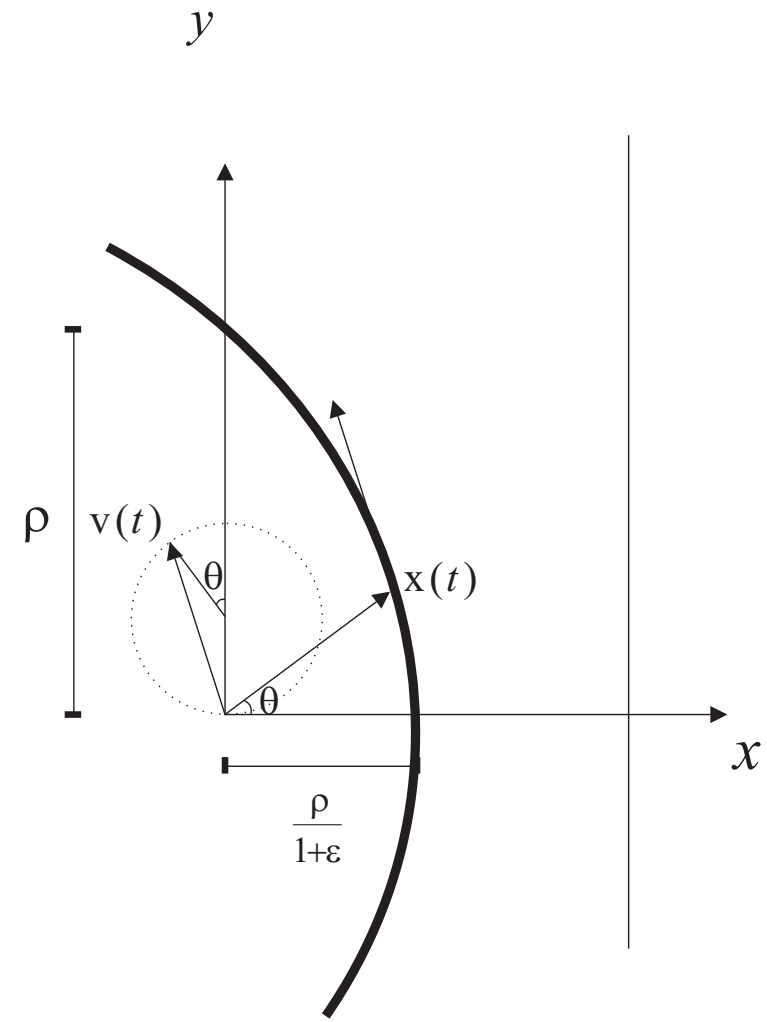

Figura 2.7: Hodógrafo do caso $E=0$, note que o círculo de velocidades é percorrido a menos de um ponto.

\subsection{Regularização}

Se não é feita a hipótese de independência linear das condições iniciais existem certos problemas com singularidades nas soluções do problema de Kepler. Estas são soluções com momento angular nulo, que correspondem a um corpo em queda sobre o outro. Em verdade, o campo hamiltoniano gerado por 2.2 não é completo. Contudo, existem procedimentos que nos permitem estudar tais soluções. Com uma regularização apropriada a singularidade em $|q|=0$ pode ser removida.

Vamos estudar primeiramente o caso unidimensional. Assim consideraremos o problema de um corpo caindo para a origem de acordo com a lei de Newton. Então a equação de movimento tem a seguinte forma

$$
\ddot{x}=-\frac{k}{|x|^{2}},
$$


cuja Hamiltoniana é dada por

$$
h=\frac{|\dot{x}|^{2}}{2}-\frac{k}{|x|} .
$$

Sem perda de generalidade vamos considerar também $x>0$.

Vamos introduzir uma nova variável independente, com o intuito de expandir a escala de tempo à medida que $x \rightarrow 0$, isto é, quanto mais próximo da origem mais devagar as mudanças nesta variável. Isto pode ser feito pela seguinte

$$
d t=|x| d s, \quad d s=\frac{d t}{|x|}
$$

Este $s=\int d t /|x|$ é conhecido como parâmetro de Levi-Civita.

Vamos usar a seguinte notação para a derivação em $s$,

$$
x^{\prime}=\frac{d x}{d s},
$$

é a nova velocidade. Prosseguimos escrevendo 2.29 nesta nova variável

$$
h=\frac{1}{2} \frac{\left|x^{\prime}\right|^{2}}{|x|^{2}}-\frac{k}{|x|}
$$

e realizando a seguinte mudança na variável dependente,

$$
x=\omega^{2} .
$$

Daí a velocidade e a energia ficam, respectivamente, dadas por

$$
x^{\prime}=2 \omega \omega^{\prime},
$$

e

$$
h=2 \frac{\omega^{\prime 2}}{\omega^{2}}-\frac{k}{\omega^{2}} .
$$


Donde

$$
2 \omega^{\prime 2}-h \omega^{2}=k
$$

Consideremos os casos possíveis $h<0, h=0$ e $h>0$.

No primeiro 2.30 nos leva a

$$
\frac{\omega^{\prime 2}}{\frac{k}{2}}+\frac{\omega^{2}}{\frac{k}{|h|}}=1 .
$$

que é a equação de uma elipse com centro na origem. Vemos que a excentricidade da elipse depende somente do valor de $h$, uma vez que o cruzamento da curva com o eixo $\omega^{\prime}$ sempre se dá em $\pm \sqrt{k / 2}$, vide figura $[2.8(\mathrm{a})]$.

No caso de energia nula temos retas, com velocidade $\pm \sqrt{k / 2}$, vide figura [2.8(b)].

Por fim, no caso negativo 2.30 nos leva a

$$
\frac{\omega^{\prime 2}}{\frac{k}{2}}-\frac{\omega^{2}}{\frac{k}{|h|}}=1
$$

que é a equação de ramos de hipérboles. Vemos que a excentricidade destas também depende somente do valor de $h$, uma vez que novamente o cruzamento da curva com o eixo $\omega^{\prime}$ sempre se dá $\mathrm{em} \pm \sqrt{k / 2}$, vide figura $[2.8(\mathrm{c})]$.

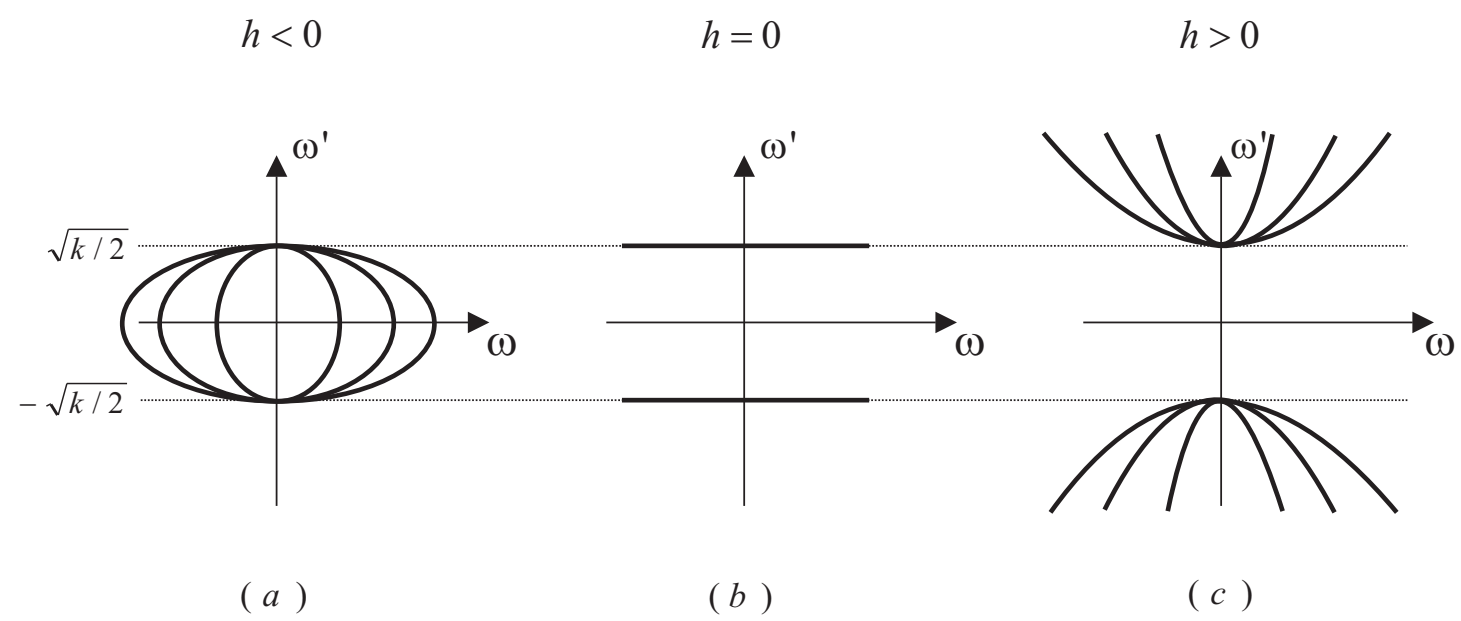

Figura 2.8: Movimento regularizado para os vários valores de energia.

O importante neste tratamento é vermos que a singularidade foi regularizada, isto é, o corpo 
cai para origem e quando chega até ela o faz com velocidade finita $\omega=0 \Rightarrow \omega^{\prime}=\sqrt{k / 2}$.

Um caso bastante interessante de regularização foi feito por Jünger Moser em [9]. Ele considerou as órbitas limitadas do problema de Kepler num espaço $n$-dimensional, isto é, considerou a Hamiltoniana

$$
H(q, p)=\frac{|p|^{2}}{2}-\frac{1}{|q|}
$$

com $q \in \mathbb{R}^{n}$ e $p \in \mathbb{R}^{n}$ sobre os níveis de energia negativos. E demonstrou que, depois de uma compactificação apropriada, a superfície de energia constante $(h<0)$ é topologicamente equivalente ao fibrado tangente unitário de uma esfera $\mathbb{S}^{n}$. Tal resultado segue no próximo teorema.

Teorema 2.3.1 Para energias negativas, $H=h<0$, o fluxo do problema de Kepler em $\mathbb{R}^{n}$ é equivalente ao fluxo geodésico sobre $\mathbb{S}^{n}$

Observação 2.3.2 Na prova a seguir queremos apenas dar uma idéia geral. Para maiores detalhes, veja [9].

Prova: Consideremos o fluxo geodésico sobre $\mathbb{S}^{n}$. Este é dado pela seguinte equação variacional vinculada,

$$
\left\{\begin{array}{l}
\delta \int\left|\xi^{\prime}\right| d s=0 \\
|\xi|=1
\end{array}\right.
$$

onde $\xi=\left(\xi_{0}, \xi_{1}, \ldots, \xi_{n}\right) \in\left(\mathbb{R}^{n+1},<,>\right),|\xi|=<\xi, \xi>^{1 / 2} \mathrm{e}^{\prime}$ continua a denotar derivação em relação a $s$. Usando a equação de Euler-Lagrange somos levados a

$$
\xi^{\prime \prime}+<\xi^{\prime}, \xi^{\prime}>\xi=0
$$

Tomando $\eta=\xi^{\prime}$ temos,

$$
\left\{\begin{array}{l}
\xi^{\prime}=\eta \\
\eta^{\prime}=-|\eta|^{2} \xi
\end{array}\right.
$$

$\operatorname{com}|\xi|=1$ e $<\xi, \eta>=0$. Assim,

$$
\Phi(\xi, \eta)=\frac{1}{2}<\eta, \eta><\xi, \xi>
$$


é uma função hamiltoniana para 2.31, desde que vale

$$
\left\{\begin{array}{l}
\xi^{\prime}=\Phi_{\eta} \\
\eta^{\prime}=-\Phi_{\xi}
\end{array} .\right.
$$

Vamos introduzir coordenadas cartesianas a este problema procedendo com uma projeção estereográfica pelo polo $(1,0, \ldots, 0)$,

$$
\pi(\xi)_{i}=x_{i}=\frac{\xi_{i}}{1-\xi_{0}}
$$

Como tratamos do fluxo sobre $\mathbb{S}^{n}$ é desejável que estendamos esta projeção de modo que a projeção de $T \mathbb{S}^{n}$ dê $\mathbb{R}^{2 n} \equiv(x, y)$, e que isto seja feito via transformações canônicas para preservar a estrutura das equações de Hamilton. Assim devemos exigir também que

$$
\sum_{m=0}^{n} \eta_{m} d \xi_{m}=\sum_{k=1}^{n} y_{k} d x_{k}
$$

Tentemos o seguinte ansatz de solução

$$
y_{k}=f(\xi, \eta) \eta_{k}+g(\xi, \eta) \xi_{k}, \quad k=1, \ldots, n,
$$

usando $2.32,2.33$ e o vínculo $|\xi|=1$ obtemos

$$
y_{k}=\left(1-\xi_{0}\right) \eta_{k}+\eta_{0} \xi_{k}
$$

As fórmulas para a inversão desta projeção, seguem

$$
\xi_{0}=\frac{|x|^{2}-1}{|x|^{2}+1}, \quad \xi_{k}=\frac{2 x_{k}}{|x|^{2}+1}, \quad k=1, \ldots, n
$$

e

$$
\eta_{0}=<x, y>, \quad \eta_{k}=\frac{|x|^{2}+1}{2} y_{k}, \quad k=1, \ldots, n
$$


A função Hamiltoniana é levada na seguinte

$$
\Phi(\xi(x, y), \eta(x, y))=F(x, y)=\frac{\left(|x|^{2}+1\right)^{2}}{8}|y|^{2}
$$

Como as transformações foram canônicas temos que as equações de Hamilton são preservadas,

$$
x^{\prime}=F_{y}, \quad y^{\prime}=-F_{x} .
$$

Vamos considerar o fluxo sobre a superfície de nível $F=1 / 2$. É fácil ver que o fluxo sobre esta superfície depende apenas do gradiente de $F$. Logo, se substituirmos $F$ por uma função de $F$, $u=u(F)$, tal que

$$
\frac{d u}{d F}\left(\frac{1}{2}\right)=1
$$

o fluxo é o mesmo. Portanto, tomemos

$$
G=\sqrt{2 F}-1=\frac{\left(|x|^{2}+1\right)}{2}|y|-1
$$

e o fluxo Hamiltoniano

$$
x^{\prime}=G_{y}, \quad y^{\prime}=-G_{x},
$$

sobre $G=0$.

Vamos considerar agora uma mudança na variável independente, análoga ao parâmetro de LeviCivita

$$
t=\int|y| d s
$$

donde obtemos

$$
\dot{x}=|y|^{-1} G_{y}, \quad \dot{y}=-|y|^{-1} G_{x} .
$$

Note que

$$
|y|^{-1} G_{y}=H_{y}
$$


e

$$
|y|^{-1} G_{x}=H_{x}
$$

com

$$
H=|y|^{-1} G-\frac{1}{2}=\frac{|x|^{2}}{2}-|y|^{-1}
$$

Finalmente, fazemos a mudança de coordenadas $(x, y) \rightarrow(-p, q)$, donde obtemos a Hamiltoniana do problema de Kepler

$$
H=\frac{|p|^{2}}{2}-\frac{1}{|q|}
$$

Assim o fluxo geodésico sobre $\mathbb{S}^{n}$ é equivalente ao do problema de Kepler em $\mathbb{R}^{n}$. Em verdade, as transformações 2.32, 2.33 e 2.35 levam o fibrado tangente unitário da esfera num espaço de fase $2 n$-dimensional, e por conseguinte levam os grandes círculos de $\mathbb{S}^{n}$ nas elipses do problema de Kepler sobre a superfície de energia $H=-1 / 2$. Segue representação pictórica da projeção estereográfica.

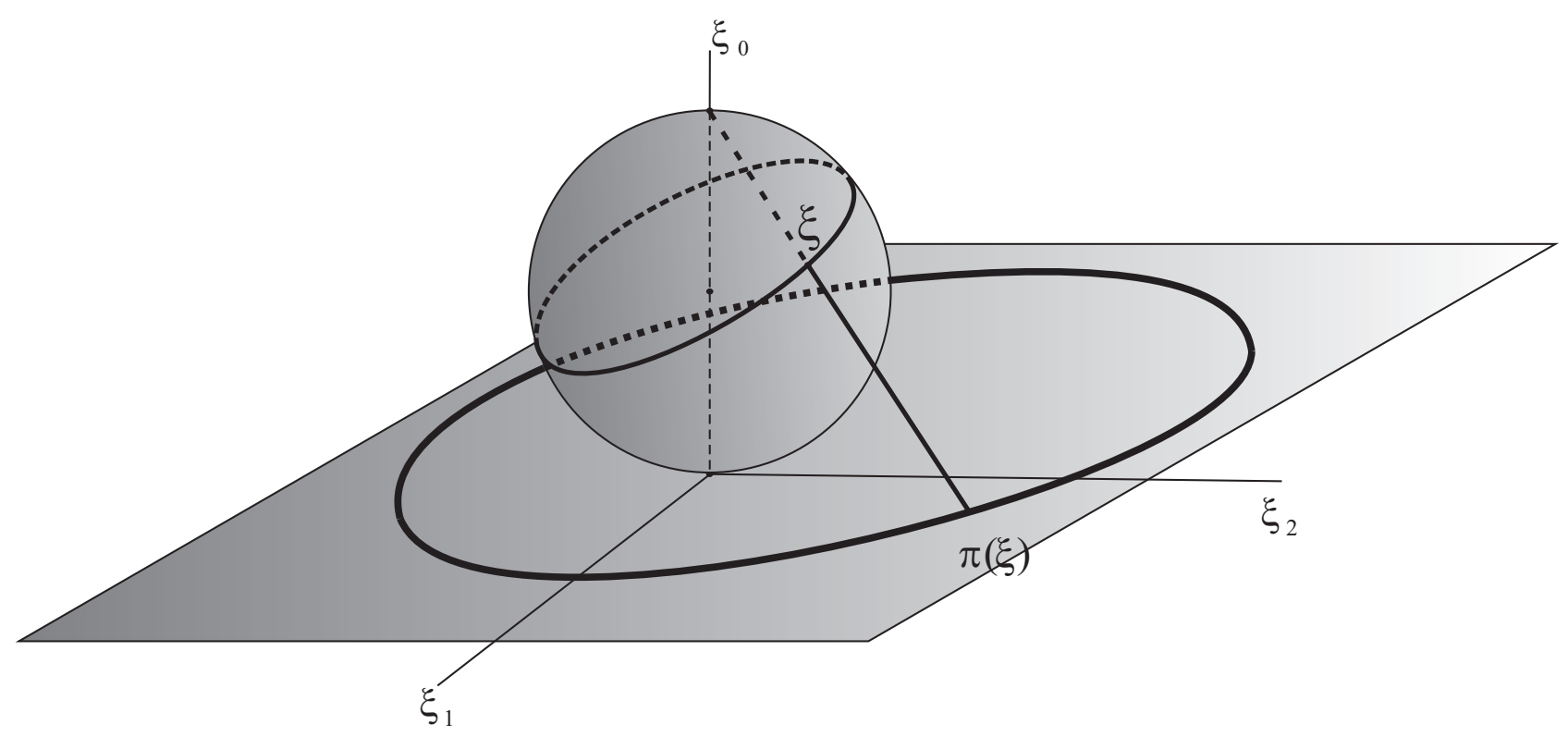

Figura 2.9: Projeção estereográfica para o caso $n=2$.

Vale ressaltar que se inserirmos o pólo norte da esfera representaremos também as órbitas de colisão do problema de Kepler, mas a projeção usada e a mudança na variável independente já nos 
dão o fluxo regularizado.

Inspirado nos trabalhos de Moser, E. A. Belbruno em [17], considerou os casos complementares ao trabalho de Moser, isto é, as órbitas ilimitadas do problema de Kepler num espaço 2-dimensional. Com uma construção bastante similar à anterior, Belbruno mostrou a equivalência entre o fluxo do problema de Kepler com energia positiva (movimento hiperbólico), e o fluxo geodésico sobre um 2hiperbolóide imerso num espaço de Lorentz 3-dimensional e que o caso de energia nula (movimento parabólico), é equivalente ao fluxo geodésico num plano. No próximo teorema mostramos o caso com energia positiva. Para maiores detalhes do caso com energia nula, vide [17].

Vamos denotar as folhas do hiperbolóide por $\mathcal{H}_{ \pm}:-\xi_{0}^{2}+\xi_{1}^{2}+\xi_{2}^{2}=-1$ ( + e - , respectivamente, se referem a folha superior e inferior do hiperbolóide), imerso num espaço de Lorentz $\mathbb{L}^{3} \equiv\left(\xi_{0}, \xi_{1}, \xi_{2}\right)$ com métrica $d s^{2}=-d \xi_{0}^{2}+d \xi_{1}^{2}+d \xi_{2}^{2}$. Façamos ainda $\xi^{+}=(1,0,0)$ e $\xi^{-}=(-1,0,0)$. A seguir consideraremos o fluxo geodésico sobre $\mathcal{H}_{+}^{+}=\left\{\left\{\mathcal{H}_{+}\right\} \backslash \xi^{+}\right\}$.

Teorema 2.3.3 Para energias positivas, $H=h>0$, o fluxo do problema de Kepler em $\mathbb{R}^{2}$ é equivalente ao fluxo geodésico sobre $\mathcal{H}_{+}^{+}$.

Observação 2.3.4 Na prova a seguir queremos apenas dar uma idéia geral. Para maiores detalhes, veja [17].

Prova: Consideremos a seguinte função

$$
<,>: \mathbb{L}^{3} \times \mathbb{L}^{3} \rightarrow \mathbb{R}
$$

dada pela seguinte regra

$$
<\alpha, \beta>=-\alpha_{0} \beta_{0}+\alpha_{1} \beta_{1}+\alpha_{2} \beta_{2}
$$

É simples ver que 2.36 define um produto interno sobre $T \mathcal{H}_{+}^{+}$e que a parametrização

$$
\xi=(\cosh u, \sinh u \cos v, \sinh u \sin v)
$$

define um referencial móvel $\left(\xi_{u}, \xi_{v}\right)$ ortogonal sobre $T \mathcal{H}_{+}^{+}$. 
Vamos agora olhar para o fluxo geodésico sobre $\mathcal{H}_{+}^{+}$. Este é dado pela seguinte equação variacional vinculada,

$$
\left\{\begin{array}{l}
\delta \int<\xi^{\prime}, \xi^{\prime}>d s=0 \\
<\xi^{\prime}, \xi^{\prime}>=-1
\end{array},\right.
$$

onde $<,>$ é a função definida em 2.36 e ' continua a denotar derivação em relação a $s$. Usando a equação de Euler-Lagrange somos levados a

$$
\xi^{\prime \prime}-<\xi^{\prime}, \xi^{\prime}>\xi=0
$$

Defina

$$
\begin{gathered}
\eta=\xi^{\prime}, \\
\Upsilon(\xi, \eta)=-\frac{1}{2}<\xi, \xi><\eta, \eta>
\end{gathered}
$$

e

$$
\Lambda=\left(\begin{array}{ccc}
-1 & 0 & 0 \\
0 & 1 & 0 \\
0 & 0 & 1
\end{array}\right)
$$

Daí temos

$$
\left\{\begin{array}{l}
\xi^{\prime}=\Lambda \Upsilon_{\eta} \\
\eta^{\prime}=-\Lambda \Upsilon_{\xi} \\
<\xi, \xi>=-1 \\
<\xi, \eta>=0
\end{array}\right.
$$

E assim o fluxo de 2.37 sobre $\Upsilon=-1 / 2$ é nosso candidato a fluxo equivalente ao do problema de Kepler.

Vamos agora construir projeções de $\mathbb{L}^{3}$ em $\left[\xi_{1}, \xi_{2}\right]$ que levarão $\mathcal{H}_{+}^{+}$em $T^{2}$, onde

$$
T^{2}=\left\{\xi \in\left[\xi_{1}, \xi_{2}\right] /<\xi, \xi>>1\right\}
$$


Para tal façamos

$$
\pi(\xi)_{i}=x_{i}=\frac{\xi_{i}}{\xi_{0}-1}
$$

Como tratamos do fluxo sobre $\mathbb{S}^{n}$ é desejável que estendamos esta projeção de modo que a projeção de $T \mathcal{H}_{+}^{+}$dê, localmente, $\mathbb{R}^{4} \equiv(x, y)$, e que isto seja feito via transformações canônicas para preservar a estrutura das equações de Hamilton. Assim devemos exigir também que

$$
\sum_{m=0}^{2} \eta_{m} d \xi_{m}=\sum_{k=1}^{2} y_{k} d x_{k}
$$

Tentemos o seguinte ansatz de solução

$$
y_{k}=f(\xi, \eta) \eta_{k}+g(\xi, \eta) \xi_{k}, \quad k=1, \ldots, n,
$$

usando $2.38,2.39$ e o vínculo $<\xi, \xi>=-1$ obtemos

$$
y_{k}=\left(\xi_{0}-1\right) \eta_{k}+\eta_{0} \xi_{k}
$$

As fórmulas para a inversão desta projeção, seguem

$$
\xi_{0}=\frac{|x|^{2}+1}{|x|^{2}-1}, \quad \xi_{k}=\frac{2 x_{k}}{|x|^{2}-1}, \quad k=1,2
$$

e

$$
\eta_{0}=<x, y>, \quad \eta_{k}=\frac{|x|^{2}-1}{2} y_{k}, \quad k=1,2
$$

A função Hamiltoniana é levada na seguinte

$$
\Upsilon(\xi(x, y), \eta(x, y))=F(x, y)=\frac{\left(|x|^{2}-1\right)^{2}}{8}|y|^{2}
$$

Como as transformações foram canônicas temos que as equações de Hamilton são preservadas,

$$
x^{\prime}=F_{y}, \quad y^{\prime}=-F_{x} .
$$




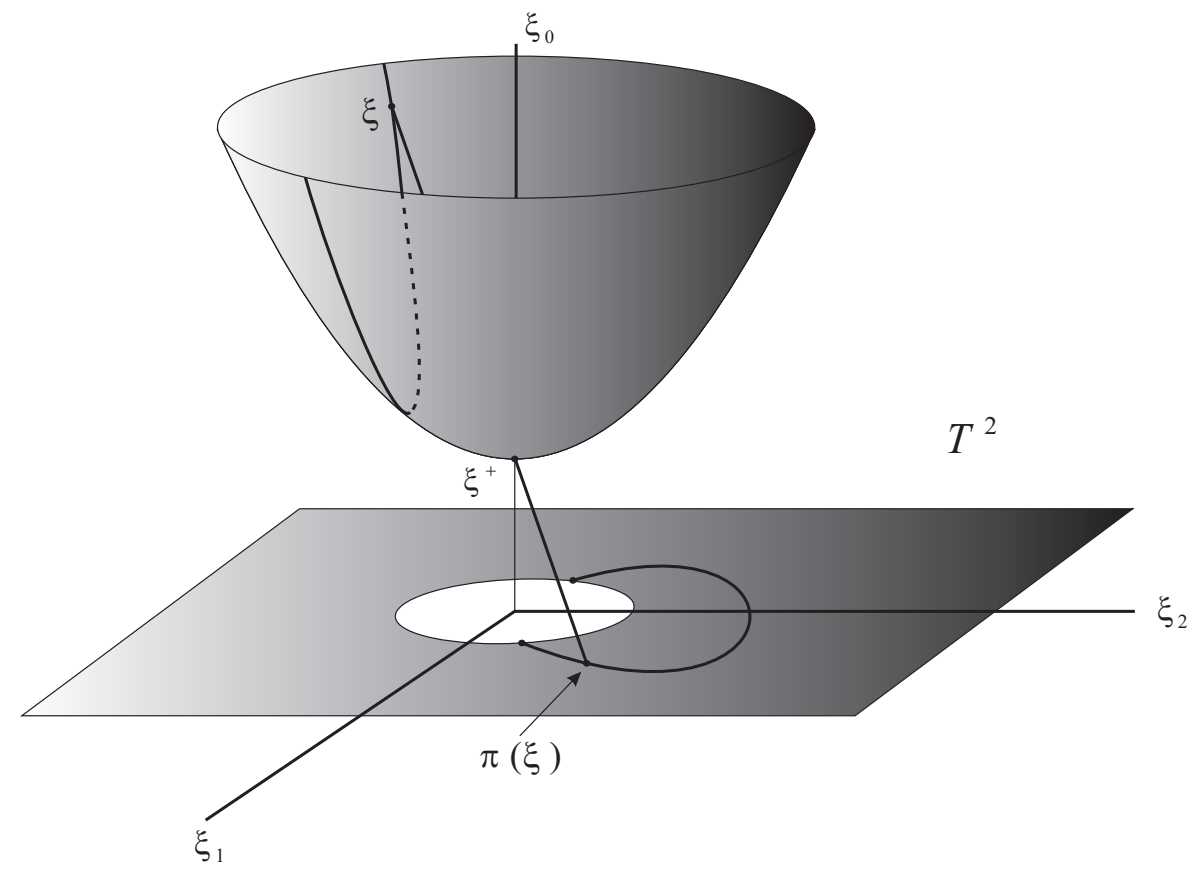

Figura 2.10: Projeção que leva $\mathcal{H}_{+}^{+}$em $T^{2}$.

Vamos considerar o fluxo sobre a superfície de nível $F=1 / 2$. Novamente, vamos substituir $F$ por uma função de $F, u=u(F)$, tal que

$$
\frac{d u}{d F}\left(\frac{1}{2}\right)=1
$$

o fluxo é o mesmo. Portanto, tomemos

$$
G=\sqrt{2 F}-1=\frac{\left(|x|^{2}+1\right)}{2}|y|-1
$$

e o fluxo Hamiltoniano

$$
x^{\prime}=G_{y}, \quad y^{\prime}=-G_{x},
$$

sobre $G=0$. 
Vamos considerar a mudança na variável independente

$$
t=\int|y| d s
$$

donde obtemos

$$
\dot{x}=|y|^{-1} G_{y}, \quad \dot{y}=-|y|^{-1} G_{x} .
$$

Note que

$$
|y|^{-1} G_{y}=H_{y}
$$

e

$$
|y|^{-1} G_{x}=H_{x}
$$

com

$$
H=|y|^{-1} G+\frac{1}{2}=\frac{|x|^{2}}{2}-|y|^{-1} .
$$

Finalmente, fazemos a mudança de coordenadas $(x, y) \rightarrow(-p, q)$, donde obtemos a Hamiltoniana do problema de Kepler

$$
H=\frac{|p|^{2}}{2}-\frac{1}{|q|}
$$

Assim o fluxo geodésico sobre $\mathcal{H}_{+}^{+}$é equivalente ao do problema de Kepler em $\mathbb{R}^{2}$. Em verdade, as transformações 2.38, 2.40 e 2.41 levam o fibrado tangente unitário de $\mathcal{H}_{+}^{+}$num espaço de fase 4-dimensional. Segue representação pictórica da projeção estereográfica. Novamente, se introduzirmos $\xi^{+}$em $\mathcal{H}_{+}^{+}$teremos a órbitas de colisão do problema de Kepler com energia positiva regularizadas.

Passaremos a alguns resultados do problema de três corpos. 


\section{Capítulo 3}

\section{Problemas de Três Corpos}

Neste capítulo vamos tratar o problema de três corpos interagindo gravitacionalmente. Na história da ciência, este foi um problema muito atacado, talvez pela proximidade com o problema solúvel de dois corpos. Muitos matemáticos, físicos e astrônomos famosos se dedicaram ao problema de três corpos gravitando e obtiveram importantes resultados. Para citar nomes, Euler, Lagrange, Jacobi, Hill, Poincaré, Levi-Chivita, Birkhoff, entre outros. Vamos ver a seguir as soluções de Euler e de Lagrange para o problema Newtoniano e a belíssima solução coreográfica em forma de Oito de Chenciner e Motgomery.

\subsection{Problema Newtoniano de 3-corpos}

Considere um sistema formado por 3 partículas de massas $m_{1}, m_{2}, m_{3}$ localizadas no espaço Euclidiano pelas coordenadas $q_{1} \in \mathbb{R}^{3}, q_{2} \in \mathbb{R}^{3}, q_{3} \in \mathbb{R}^{3}$, respectivamente. Suponha que a interação sobre a $i$-ésima partícula para com a $j$-ésima seja dada pela Lei da Gravitação Universal de Newton, conforme enunciado no capítulo 2, suponha também que seja válido o princípio da superposição. Assim, a Lagrangiana do sistema é dada por

$$
L=\frac{1}{2} \sum_{i=1}^{3} m_{i}\left|\dot{q}_{i}\right|^{2}+\sum_{1 \leq i<j \leq 3} \frac{G m_{i} m_{j}}{\left|q_{i}-q_{j}\right|} .
$$


Usando a equação de Euler-Lagrange temos

$$
\left\{\begin{array}{l}
\ddot{q}_{1}=-\frac{G m_{2}\left(q_{1}-q_{2}\right)}{\left|q_{1}-q_{2}\right|^{3}}-\frac{G m_{3}\left(q_{1}-q_{3}\right)}{\left|q_{1}-q_{3}\right|^{3}} \\
\ddot{q}_{2}=-\frac{G m_{1}\left(q_{2}-q_{1}\right)}{\left|q_{2}-q_{1}\right|^{3}}-\frac{G m_{3}\left(q_{2}-q_{3}\right)}{\left|q_{2}-q_{3}\right|^{3}} \\
\ddot{q}_{3}=-\frac{G m_{1}\left(q_{3}-q_{1}\right)}{\left|q_{3}-q_{1}\right|^{3}}-\frac{G m_{2}\left(q_{3}-q_{2}\right)}{\left|q_{3}-q_{2}\right|^{3}}
\end{array} .\right.
$$

A equação 3.2 é uma equação diferencial em $\mathbb{R}^{18}$, isto é, para resolvê-la precisamos determinar uma 6-upla de coordenadas para cada corpo. Poderíamos tentar solucionar 3.2 encontrando integrais primeiras que reduzissem seu número de graus de liberdade. Porém as integrais usadas no problema de dois corpos não seriam suficientes, pois sendo elas apenas 10 levariam a equação 18-dimensional em outra 8-dimensional que ainda não poderia ser resolvida diretamente. Portanto, deve-se encontrar outro método ou outras integrais primeiras o que, em verdade, não é possível visto que pelo teorema de Bruns o problema de 3-corpos tem apenas 10 integrais primeiras algébricas, as mesmas do problema de 2-corpos, vide o teorema 4.1.1 no próximo capítulo.

Neste ponto de nosso estudo precisamos impor uma restrição que nos ajuda a diminuir os graus de liberdade do problema. Vamos supor que o movimento dos três corpos se dê em um plano fixo. Isto leva 3.2 numa equação 12-dimensional que tem 6 integrais primeiras, ou seja, ainda não pode ser resolvida diretamente.

Supondo que estamos trabalhando no referencial do centro de massa, temos

$$
m_{1} q_{1}+m_{2} q_{2}+m_{3} q_{3}=0
$$

Consideremos então que seja planar o movimento dos corpos e a seguinte mudança de coordenadas [19], vide figura 3.1,

$$
\left\{\begin{array}{l}
s_{1}=q_{3}-q_{2} \\
s_{2}=q_{1}-q_{3} \\
s_{3}=q_{2}-q_{1} \\
s_{1}+s_{2}+s_{3}=0
\end{array}\right.
$$




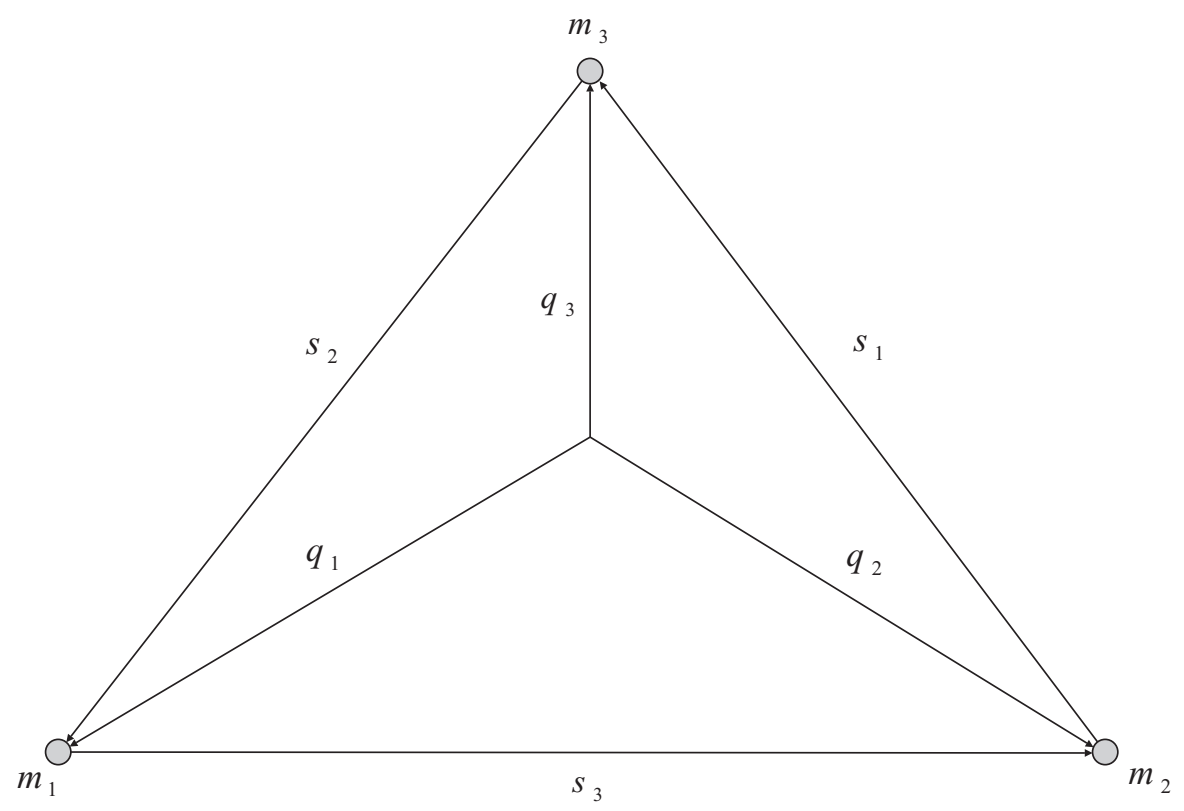

Figura 3.1: Representação das coordenadas relativas.

Assim usando 3.4 o sistema 3.2 fica na seguinte forma,

$$
\left\{\begin{array}{c}
\ddot{s_{1}}=-\frac{G M}{\left|s_{1}\right|^{3}} s_{1}+G m_{1} s_{T} \\
\ddot{s_{2}}=-\frac{G M}{\left|s_{2}\right|^{3}} s_{2}+G m_{2} s_{T} \\
\ddot{s_{3}}=-\frac{G M}{\left|s_{3}\right|^{3}} s_{3}+G m_{3} s_{T} \\
s_{T}=\frac{s_{1}}{\left|s_{1}\right|^{3}}+\frac{s_{2}}{\left|s_{2}\right|^{3}}+\frac{s_{3}}{\left|s_{3}\right|^{3}}
\end{array} .\right.
$$

onde $M$ é a massa total do sistema. Usando 3.4 e 3.3 vemos que

$$
\left\{\begin{array}{l}
q_{1}=\frac{m_{2} s_{3}-m_{3} s_{2}}{M} \\
q_{2}=\frac{m_{3} s_{1}-m_{1} s_{3}}{M} \\
q_{3}=\frac{m_{1} s_{2}-m_{2} s_{1}}{M}
\end{array} .\right.
$$

Passemos agora aos resultados de Euler de 1767, vide [20] e [19].

Teorema 3.1.1 Considere um problema planar de três corpos interagindo gravitacionalmente. Então, existe uma solução tal que os corpos permanecem sobre uma reta a cada instante do movi- 
mento.

Prova: Para este caso a Lagrangiana é dada por $3.1 \mathrm{com} q_{i} \in \mathbb{R}^{2}$, o que leva ainda às equações de Euler-Lagrange da forma 3.2. Considere o uso da mudança de coordenadas 3.4, isto conduz ao sistema 3.5. Dizer que as partículas permanecem sobre uma mesma reta a cada instante é análogo a dizer que as coordenadas relativas respeitam à condição

$$
\left\{\begin{array}{l}
s_{2}=\psi s_{1} \\
s_{3}=-(1+\psi) s_{1}
\end{array}\right.
$$

onde $\psi$ é uma função constante de sinal definido, positiva por simplicidade.

Diferenciando duas vezes a primeira equação de 3.6 e agrupando com as duas primeiras equações de 3.5 temos

$$
\ddot{\psi} s_{1}+2 \dot{\psi} \dot{s}_{1}=G\left[M \psi-\frac{M}{\psi^{2}}+\left(m_{2}-m_{1} \psi\right)\left(1+\frac{1}{\psi^{2}}-\frac{1}{(1+\psi)^{2}}\right)\right] \frac{s_{1}}{\left|s_{1}\right|^{3}}
$$

mas como,

$$
\dot{\psi}=0
$$

Temos que o lado direito de 3.7 deve se anular, ou seja, o polinômio

$$
\left(m_{2}+m_{3}\right) \psi^{5}+\left(3 m_{2}+2 m_{3}\right) \psi^{4}+\left(3 m_{2}+m_{3}\right) \psi^{3}-\left(3 m_{1}+m_{3}\right) \psi^{2}-\left(3 m_{1}+2 m_{3}\right)-\left(m_{1}+m_{3}\right),
$$

deve ter uma raiz positiva. De fato, pela regra dos sinais de Decartes, vemos que este polinômio tem uma raiz positiva. Assim, com este $\psi$ podemos voltar à equação 3.5 e subtrair da primeira equação multiplicada por $m_{3}$ a terceira multiplicada por $m_{1}$, donde obtemos

$$
\ddot{s_{1}}=-G M\left\{\frac{m_{1}+m_{3}(1+\psi)^{2}}{(1+\psi)^{2}\left[m_{3}+m_{1}(1+\psi)\right]}\right\} \frac{s_{1}}{\left|s_{1}\right|^{3}}
$$

A equação 3.8 tem a mesma forma da equação 2.5 acima. Assim usando métodos análogos podemos 
resolver 3.8 e obter, em coordenadas polares, um resultado da forma

$$
s_{1}^{r}=\frac{\chi}{1+\delta \cos \theta}
$$

onde

$$
\chi=\frac{J_{1}^{2}\left\{(1+\psi)^{2}\left[m_{3}+m_{1}(1+\psi)\right]\right\}}{G M\left[m_{1}+m_{3}(1+\psi)^{2}\right]}
$$

$\mathrm{e}$

$$
\delta=\sqrt{\frac{2 E_{1} J_{1}^{2}}{\left\{\frac{G M\left[m_{1}+m_{3}(1+\psi)^{2}\right]}{(1+\psi)^{2}\left[m_{3}+m_{1}(1+\psi)\right]}\right\}^{2}}+1},
$$

sendo $J_{1}$ a norma de uma integral primeira análoga ao momento angular, mas para a coordenada relativa $s_{1}, E_{1}$ seria a energia, mas da coordenada relativa $s_{1}$ e $s_{1}^{r}$ indica a coordenada polar radial. Daí usando 3.6, obtemos

$$
\left\{\begin{array}{l}
s_{1}=\frac{\chi}{1+\delta \cos \theta} \widehat{s_{1}} \\
s_{2}=\frac{\chi \psi}{1+\delta \cos \theta} \widehat{s_{1}} \\
s_{3}=\frac{-(1+\psi) \chi}{1+\delta \cos \theta} \widehat{s_{1}}
\end{array}\right.
$$

onde $\widehat{s_{1}}$ é a indicação da mudança de direção de $s_{1}$ no plano.

Por fim, usando as expressões para a inversão da mudança de coordenadas nos vem

$$
\left\{\begin{array}{l}
q_{1}=\frac{-\chi\left[m_{2}+\psi\left(m_{2}+m_{3}\right)\right]}{M(1+\delta \cos \theta)} \widehat{s_{1}} \\
q_{2}=\frac{\chi\left[m_{3}+m_{1}(1+\psi)\right]}{M(1+\delta \cos \theta)} \widehat{s_{1}} \\
q_{3}=\frac{\chi\left(m_{1} \psi-m_{2}\right)}{M(1+\delta \cos \theta)} \widehat{s_{1}}
\end{array} .\right.
$$

A expressão 3.9 é chamada solução gráfica, pois dela temos informação apenas sobre as configurações dos corpos no espaço.

Se nas condições iniciais temos $\delta=0$, teremos uma solução onde os corpos mantêm as distâncias mútuas fixas e permanecem sobre uma reta a cada instante, vide figura 3.2. Essa solução é chamada de equilíbrio relativo.

Com outras condições iniciais podemos ter elipses, parábolas ou hipérboles dependendo dos valores de energia e das massas. Como ilustração colocamos o caso elíptico, vide figura 3.3. 


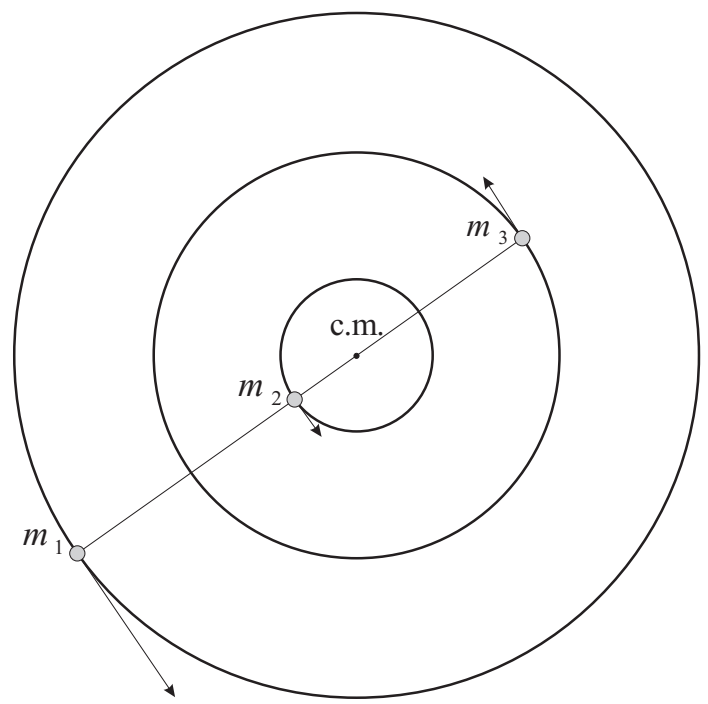

Figura 3.2: Representação da solução de equilíbrio relativo de Euler. Aqui $m_{1}: m_{2}: m_{3}=2: 3: 4$.

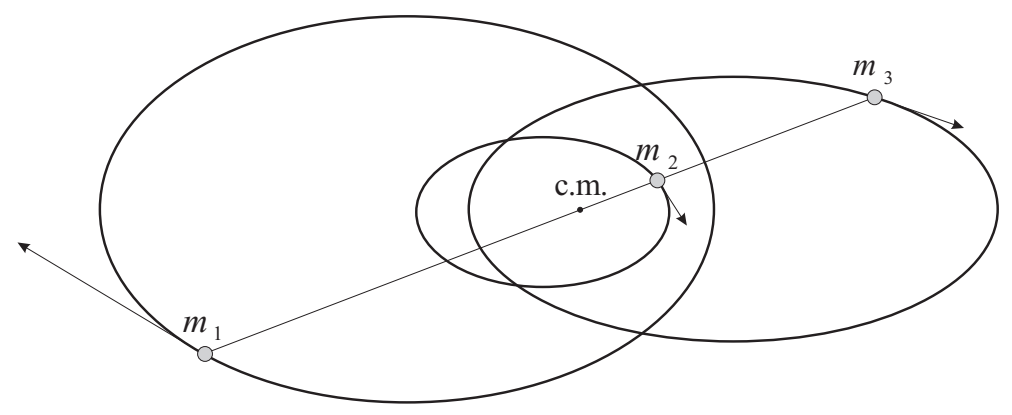

Figura 3.3: Representação da solução de Euler. Aqui $m_{1}: m_{2}: m_{3}=2: 3: 4$.

Passemos agora aos resultados de Lagrange de 1772, vide [21] e [19].

Teorema 3.1.2 Considere um problema planar de três corpos interagindo gravitacionalmente. Então, existe uma solução tal que os corpos permanecem sobre os vértices de um triângulo eqüilátero a cada instante do movimento.

Prova: Para este caso a Lagrangiana é dada por $3.1 \mathrm{com} q_{i} \in \mathbb{R}^{2}$, o que leva ainda às equações de Euler-Lagrange da forma 3.2. Considere o uso da mudança de coordenadas 3.4, isto conduz ao sistema 3.5. Notemos que em 3.5 se $s_{T}=0$ teremos equações desacopladas, vamos então supor que tal condição seja válida. Temos que $s_{T}=0$ em duas situações. Primeira, quando $\left|s_{1}\right|=\left|s_{2}\right|=\left|s_{3}\right|$, 
que nos leva a uma solução com os três corpos equidistantes. Segunda, quando os três corpos estão sobre uma mesma reta,que é a solução de Euler com um valor fixo para $\psi$. Logo, vamos nos ater à solução com corpos equidistantes. Desta feita vamos ter a 3.5 desacoplada na seguinte forma,

$$
\left\{\begin{array}{c}
\ddot{s_{1}}=-\frac{G M}{\left|s_{1}\right|^{3}} s_{1} \\
\ddot{s_{2}}=-\frac{G M}{\left|s_{2}\right|^{3}} s_{2} \\
\ddot{s_{3}}=-\frac{G M}{\left|s_{3}\right|^{3}} s_{3}
\end{array} .\right.
$$

As equações 3.10 estão na mesma forma de 2.5. Estão com métodos similares aos usados acima podemos encontrar suas soluções. Assim temos

$$
\left\{\begin{array}{l}
s_{1}=\frac{\kappa_{1}}{1+\delta_{1} \cos \theta} \widehat{s_{1}} \\
s_{2}=\frac{\kappa_{2}}{1+\delta_{2} \cos \theta} \widehat{s_{2}} \\
s_{3}=\frac{\kappa_{3}}{1+\delta_{3} \cos \theta} \widehat{s_{3}}
\end{array}\right.
$$

onde

$$
\kappa_{i}=\frac{J_{i}}{G M}
$$

$\mathrm{e}$

$$
\delta_{i}=\sqrt{\frac{2 E_{i} J_{i}^{2}}{M}+1},
$$

sendo $J_{i}$ a norma de uma integral primeira análoga ao momento angular, mas para a coordenada relativa $s_{i}, E_{i}$ seria a energia, mas da coordenada relativa $s_{i}$ e $\widehat{s_{1}}$ indica a mudança de direção de $s_{1}$ no plano.

Lembrando ainda que para a solução eqüilátera temos

$$
\widehat{s_{2}}=\left[\begin{array}{cc}
-1 / 2 & \sqrt{3} / 2 \\
-\sqrt{3} / 2 & -1 / 2
\end{array}\right] \widehat{s_{1}},
$$




$$
\begin{gathered}
\widehat{s_{3}}=\left[\begin{array}{cc}
-1 / 2 & -\sqrt{3} / 2 \\
\sqrt{3} / 2 & -1 / 2
\end{array}\right] \widehat{s_{1}}, \\
\delta_{1}=\delta_{2}=\delta_{3},
\end{gathered}
$$

e

$$
\kappa_{1}=\kappa_{2}=\kappa_{3}
$$

Por fim, usando as fórmulas para inversão da mudança de coordenadas recebemos.

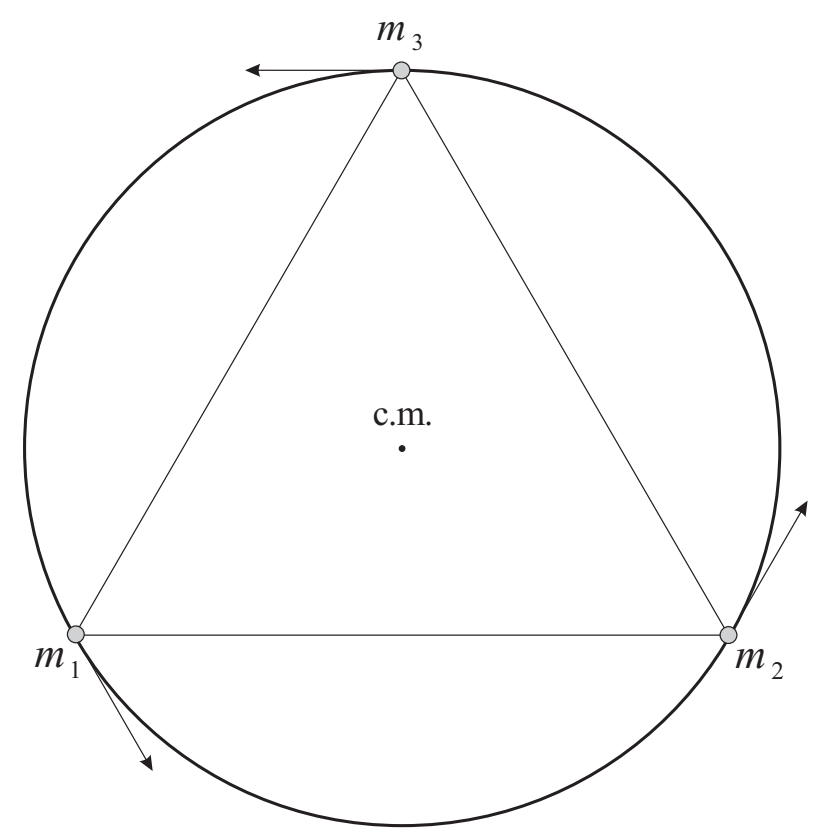

Figura 3.4: Representação da solução de equilíbrio relativo de Lagrange. Aqui devemos ter $m_{1}=m_{2}=m_{3}$.

$$
q_{1}=\frac{\kappa_{1}}{M\left(1+\delta_{1} \cos \theta\right)}\left[\begin{array}{ll}
\frac{\left(m_{3}-m_{2}\right)}{2} & \frac{\sqrt{3}\left(m_{3}+m_{2}\right)}{2} \\
\frac{\sqrt{3}\left(m_{3}-m_{2}\right)}{2} & \frac{\left(m_{3}-m_{2}\right)}{2}
\end{array}\right] \widehat{s_{1}},
$$




$$
\begin{gathered}
q_{2}=\frac{\kappa_{2}}{M\left(1+\delta_{2} \cos \theta\right)}\left[\begin{array}{ll}
\frac{\left(2 m_{3}+m_{1}\right)}{2} & \frac{\sqrt{3} m_{1}}{2} \\
\frac{\sqrt{3} m_{1}}{2} & \frac{\left(2 m_{3}+m_{1}\right)}{2}
\end{array}\right] \widehat{s_{1}} \\
q_{3}=\frac{\kappa_{3}}{M\left(1+\delta_{3} \cos \theta\right)}\left[\begin{array}{ll}
-\frac{\left(m_{1}+2 m_{2}\right)}{2} & \frac{\sqrt{3} m_{1}}{2} \\
-\frac{\sqrt{3} m_{1}}{2} & -\frac{\left(m_{1}+2 m_{2}\right)}{2}
\end{array}\right] \widehat{s_{1}}
\end{gathered}
$$

Como antes, estas são chamadas de soluções gráficas, pois carregam apenas as informações sobre o hodógrafo das massas no plano.

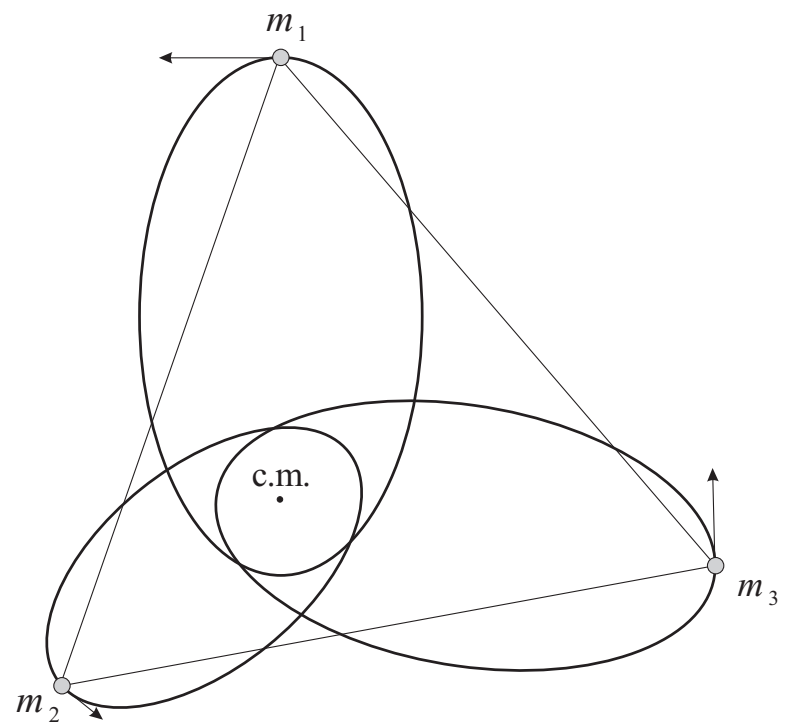

Figura 3.5: Representação da solução de Lagrange. Aqui devemos ter $m_{1}<m_{2}<m_{3}$.

Se nas condições iniciais tivermos $\delta_{i}=0$, teremos novamente uma solução em que a distância entre os corpos é preservada, vide figura 3.4. Esta solução também é chamada de equilíbrio relativo. Para soluções com $\kappa_{i} \neq 0$, teremos elipses, parábolas ou hipérboles dependendo dos valores da energia. Como ilustração colocamos aqui o caso elíptico, vide figura 3.5. 


\subsection{Uma Solução Memorável para o Problema de Três Corpos}

Uma solução particular bastante interessante do problema de três corpos é obtida no caso de três massas iguais, fazendo uma órbita em forma de Oito, vide figura 3.6, descoberta numericamente por C. Moore em 1993 [22]. Em 2001 A. Chenciner e R. Montgomery [23] redescobriram o Oito e provaram sua existência. Segue descrição deste problema.

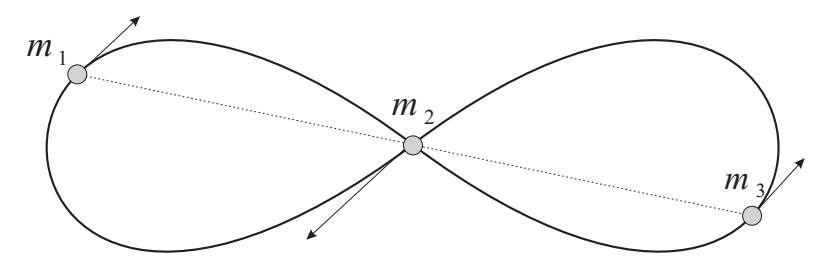

Figura 3.6: A Órbita em oito de Chenciner e Montgomery.

Considere um problema planar de três corpos, cada um com massa unitária, que interagem pela lei da Gravitação Universal de Newton. Para tal sistema teremos a seguinte função principal de Lagrange

$$
L=\frac{1}{2}\left(\left|\dot{q}_{1}\right|^{2}+\left|\dot{q}_{2}\right|^{2}+\left|\dot{q}_{3}\right|^{2}\right)+\frac{1}{\left|q_{1}-q_{2}\right|}+\frac{1}{\left|q_{1}-q_{3}\right|}+\frac{1}{\left|q_{2}-q_{3}\right|}, \quad q_{i} \in \mathbb{R}^{2}
$$

onde consideramos, por simplicidade, $G=1$, vide 4.0.13, isto é, o espaço de configuração com coordenadas baricêntricas é dado por

$$
X=\left\{q=\left(q_{1}, q_{2}, q_{3}\right) \in \mathbb{R}^{6} / q_{1}+q_{2}+q_{3}=0\right\}
$$

\subsubsection{Construção do Espaço de Triângulos Orientados.}

Primeiramente notemos que 3.11 leva, em princípio, a uma equação em $\mathbb{R}^{12}$. No espaço de configuração é conhecido que após a redução com as simetrias por translação e rotação o espaço de configuração do problema de três corpos é uma variedade de dimensão três homeomorfa a $\mathbb{R}^{3}$, vide [25] página 4. Vamos estudar a cinemática de soluções particulares as quais tem momento angular total nulo.

O chamado espaço de triângulos orientados, vide [24], foi colocado primeiramente por Richard 
Moeckel em [25], e por ele chamado de esfera de forma, vide figura 3.13. Mostraremos aqui duas possíveis construções desta esfera, a primeira delas usando a fórmula de Herón de Alexandria. Considerando $\mathcal{T}$ o triângulo formado pelos três corpos, vide figura 3.7. A fórmula de Herón fica

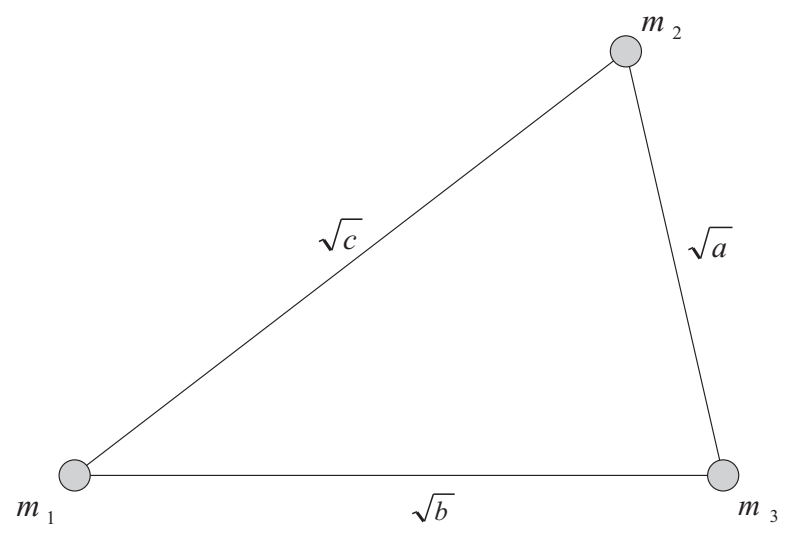

Figura 3.7: Triângulo $\mathcal{T}$ formado pelos três corpos.

dada por

$$
S^{2}=p(p-\sqrt{a})(p-\sqrt{b})(p-\sqrt{c})
$$

onde $S$ e $p$ representam, respectivamente, a área e o semi-perímetro de $\mathcal{T}$. A expressão 3.12 pode ser escrita na forma

$$
S^{2}=\frac{1}{16}\left(2 a b+2 a c+2 b c-a^{2}-b^{2}-c^{2}\right) .
$$

Daqui por diante, nesta seção, identificaremos $m_{i}=i$, para facilitar as notações. Consideraremos também que a terna $(a, b, c)$ representa todos os triângulos cujos lados têm essas medidas a menos de reflexões. Para ser mais precisos, tomaremos o conjunto dos triângulos no plano quocientado pela rotação e translação de triângulos. Assim cada ponto neste espaço representa duas classes de triângulos, vide figura 3.10 .

Proposição 3.2.1 Nas condições descritas acima temos

$$
\left\{\begin{array}{l}
\left|q_{1}\right|^{2}+\left|q_{2}\right|^{2}+\left|q_{3}\right|^{2}=1 \\
q_{1}+q_{2}+q_{3}=0
\end{array} \Leftrightarrow a+b+c=3 .\right.
$$


Prova: Observemos a figura abaixo,

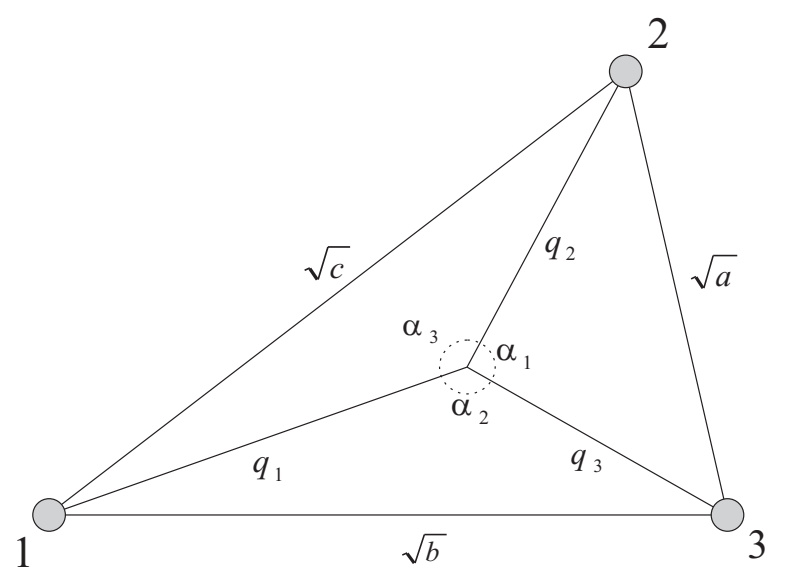

Figura 3.8: Coordenadas e ângulos em $\mathcal{T}$.

Notemos que são válidas as seguintes relações,

$$
\left\{\begin{array}{l}
a=q_{2}^{2}+q_{3}^{2}-2 q_{2} q_{3} \cos \alpha_{1} \\
b=q_{1}^{2}+q_{3}^{2}-2 q_{1} q_{3} \cos \alpha_{2} \\
c=q_{1}^{2}+q_{2}^{2}-2 q_{1} q_{1} \cos \alpha_{3}
\end{array}\right.
$$

ou equivalentemente,

$$
\left\{\begin{array}{l}
a=\left(q_{2}-q_{3}\right) \bullet\left(q_{2}-q_{3}\right) \\
b=\left(q_{1}-q_{3}\right) \bullet\left(q_{1}-q_{3}\right) \\
c=\left(q_{1}-q_{2}\right) \bullet\left(q_{1}-q_{2}\right)
\end{array}\right.
$$

onde • indica o produto interno usual do plano.

Somando as equações de 3.13 e reagrupando temos

$$
a+b+c=q_{1} \bullet\left(2 q_{1}-q_{2}-q_{3}\right)+q_{2} \bullet\left(2 q_{2}-q_{1}-q_{3}\right)+q_{3} \bullet\left(2 q_{3}-q_{1}-q_{2}\right)
$$

ou ainda,

$$
a+b+c=3 q_{1} \bullet q_{1}+3 q_{2} \bullet q_{2}+3 q_{3} \bullet q_{3},
$$


que é nos dá

$$
\frac{a+b+c}{3}=\left|q_{1}\right|^{2}+\left|q_{2}\right|^{2}+\left|q_{3}\right|^{2}
$$

Isto confirma o resultado do enunciado.

Assim no espaço Euclidiano das ternas $(a, b, c)$, tomar o momento de inércia unitário é o mesmo que fazer a interseção do primeiro octante com o plano

$$
a+b+c=3
$$

vide figura 3.9 .

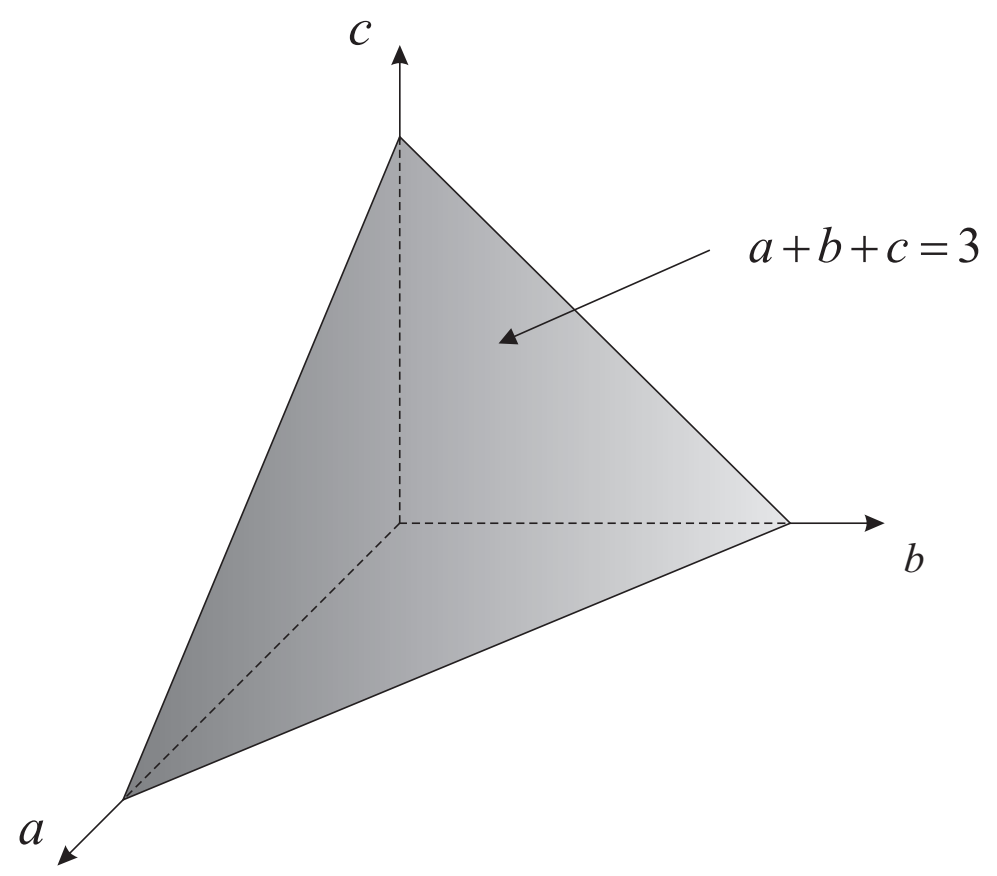

Figura 3.9: Plano equivalente ao momento de inércia unitário.

Vamos agora localizar alguns triângulos do espaço de configuração associados com esse plano. O ponto de interseção com a reta $a=b=c$ é $(1,1,1)$ e dá a configuração do triângulo eqüilátero, que corresponde à solução de Lagrange, mostrada no teorema 3.1.2. O ponto médio de $c=3-b$, 
com $a=0$, é a colisão binária $C_{1}(2 \operatorname{com} 3)$, o ponto médio de $c=3-a, \operatorname{com} b=0$, é $C_{2}(1 \operatorname{com} 3)$ e, por fim, o ponto médio de $b=3-a$, com $c=0$, é $C_{3}(2$ com 1$)$, vide figura 3.10.

Proposição 3.2.2 A área dos triângulos é constante nas interseções desse plano, $a+b+c=3$, com cada casca esférica

$$
(a-1)^{2}+(b-1)^{2}+(c-1)^{2}=r^{2},
$$

$\operatorname{com} r \leq \sqrt{3 / 2}$

Prova: Vamos tomar a segunda forma da fórmula de Herón,a expressão do plano que intercepta o octante e a expressão da casca e colocar na forma de um sistema não linear, o qual segue

$$
\left\{\begin{array}{l}
S^{2}=\frac{1}{16}\left(2 a b+2 b c+2 a c-a^{2}-b^{2}-c^{2}\right) \\
a+b+c=3 \\
(a-1)^{2}+(b-1)^{2}+(c-1)^{2}=r^{2}
\end{array} .\right.
$$

Expandindo a terceira equação de 3.14, temos

$$
a^{2}+b^{2}+c^{2}-2 a-2 b-2 c+3=r^{2},
$$

podemos também reescrever a primeira equação de 3.14 da seguinte maneira

$$
S^{2}=\frac{1}{16}\left[(a+b+c)(a+b+c)-2\left(a^{2}+b^{2}+c^{2}\right)\right] .
$$

Inserindo 3.15 em 3.16, temos

$$
S^{2}=\frac{1}{16}\left[(a+b+c)(a+b+c)-2\left(r^{2}+2 a+2 b+2 c-3\right)\right] .
$$

Usando agora a segunda equação de 3.14 sai

$$
S^{2}=\frac{1}{16}\left(3-2 r^{2}\right)
$$


Assim quando olhamos na figura 3.10, vemos que a área dos triângulos decresce à medida que nos afastamos de $(1,1,1)$, sobre o plano $a+b+c=3$ e que esta área se torna zero quando estamos nas bordas, ou seja, quando $r=\sqrt{3 / 2}$.

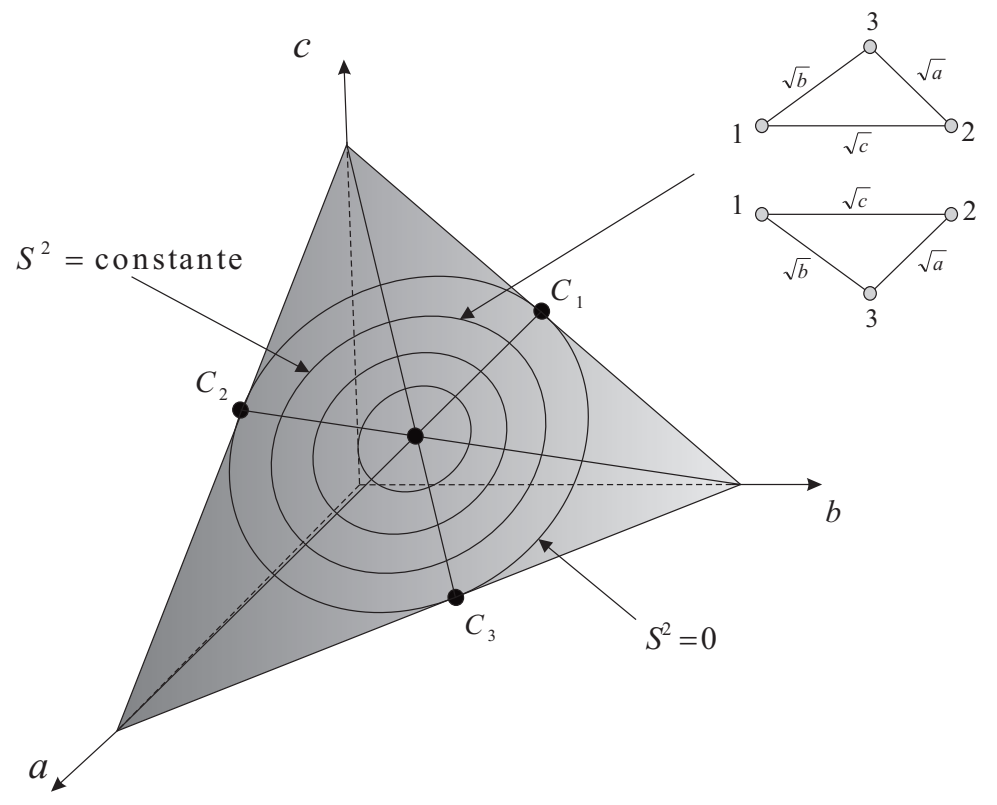

Figura 3.10: Representação de alguns pontos no plano.

Consideremos agora as seguintes operações:

1. Excluir os pontos do plano $a+b+c=3$, que distam de $(1,1,1)$ com um raio maior que raiz quadrada de três meios, de modo que seja obtido um disco de raio $R=\sqrt{3 / 2}$, onde moram todas as possíveis configurações de triângulos com momento de inércia unitário, vide figura 3.11

2. Como a cada ponto desse disco estão associadas duas classes de triângulos, de orientações distintas, vamos separar essas classes de uma forma conveniente. Estabelecemos a correspondência entre este disco e os dois hemisférios, com a convenção configurações positivamente orientadas no hemisfério norte e as negativas no hemisfério sul, vide figura 3.12;

3. Justapor estes dois hemisférios com as devidas posições correspondentes no equador. 


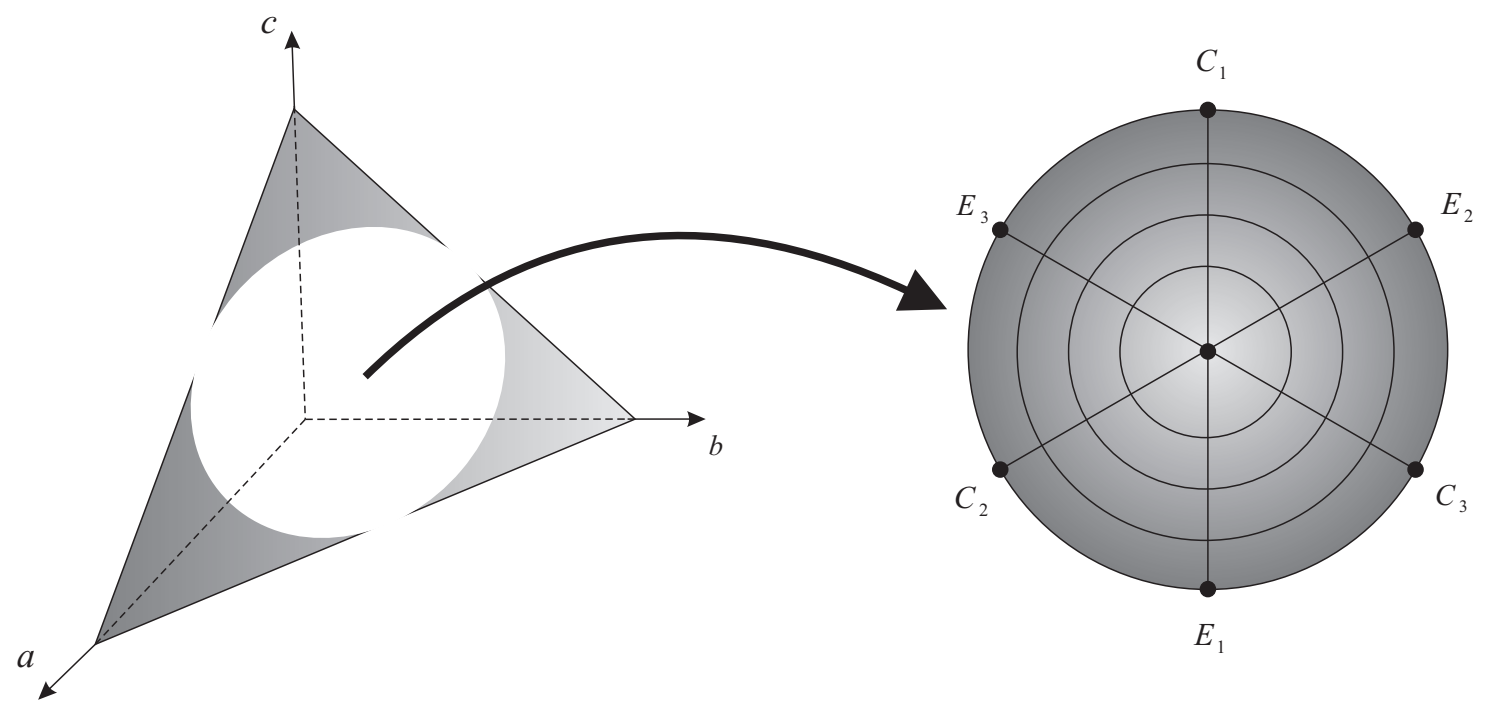

Figura 3.11: Ilustração da retirada do disco de $a+b+c=3$.

Assim temos construída a esfera de forma, ou o espaço dos triângulos orientados, como o descrito acima. Nessa representação a colisão total é

$$
a=b=c=0,
$$

e as colisões binárias são indicadas da seguinte maneira

$$
\left\{\begin{array}{rl}
C_{1} & \equiv(2 \operatorname{com} 3) \equiv[a=0] \\
C_{2} & \equiv(1 \operatorname{com} 3) \equiv[b=0] \\
C_{3} & \equiv(1 \operatorname{com} 2) \equiv[c=0]
\end{array} .\right.
$$

As soluções de Euler, descritas no teorema 3.1.1 são encontradas no disco antípodas às colisões binárias. Como tanto as colisões quanto as configurações de Euler são invariantes pela reflexão através do equador devem ser levadas nos mesmos pontos. Assim tomando a aplicação antípoda na própria esfera, teremos

$$
C_{i} \mapsto E_{i},
$$

onde é feita a convenção $E_{i} \equiv$ configuração da solução de Euler com o $i$-ésimo corpo entre os outros 
dois.

Notemos que o segmento meridiano que liga $C_{i}$ a $E_{i}$ representa uma família de triângulos isósceles. Chamaremos cada um desses meridianos de $I S O_{i}$, vide figura 3.12. Os pólos dos hemisférios correspondem à solução de Lagrange. Através desta configuração fixaremos uma orientação para os triângulos sobre a esfera dizendo que o pólo norte tem orientação positiva. Assim chamaremos este ponto de $L A G_{+}$. Por mimetismo, chamaremos o pólo sul de $L A G_{-}$. Se sobre a esfera
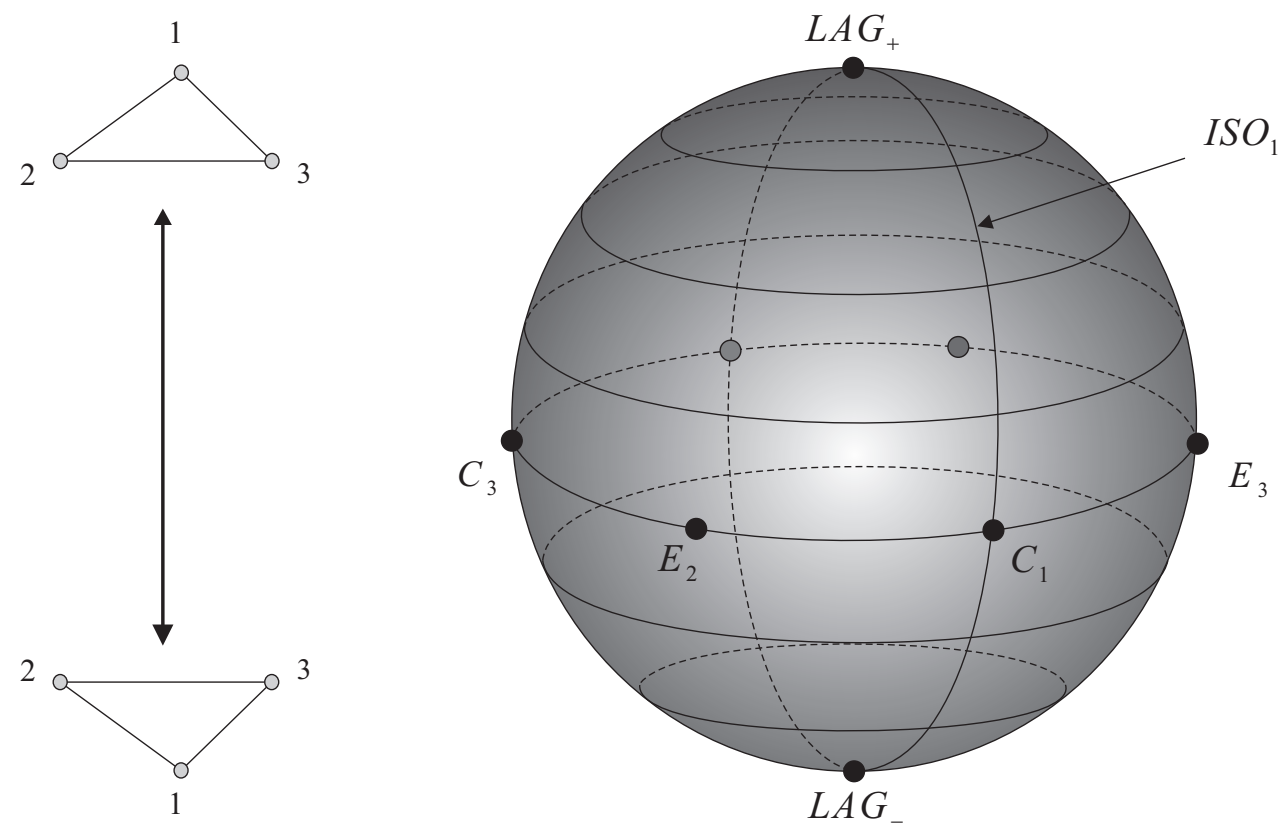

Figura 3.12: Espaço dos triângulos orientados.

de forma imaginamos coordenadas esféricas, podemos entender a coordenada azimutal como sendo relacionada à área dos triângulos; a coordenada axial variando sobre uma dada latitude fixa como mudanças nos triângulos que preservem sua área; por fim, a coordenada radial estaria relacionada com o valor da soma dos lados do triângulo.

Passemos agora a uma construção do espaço de triângulos orientados, considerando agora a complexificação das coordenadas $q_{i}$ e usando duas funções conhecidas na literatura. A função de 
mudança de coordenadas de Jacobi, mas com uma mudança de escala, a qual segue

$$
\mathfrak{J}\left(q_{1}, q_{2}, q_{3}\right)=\left(z_{1}, z_{2}\right)=\left(\frac{1}{\sqrt{2}}\left(q_{3}-q_{2}\right), \sqrt{\frac{2}{3}}\left(q_{1}-\frac{1}{2}\left(q_{2}+q_{3}\right)\right)\right) .
$$

a primeira coordenada de Jacobi foi omitida pois já estamos no referencial do centro de massa. A diferença desta aplicação para as coordenadas de Jacobi está nos fatores que multiplicam as coordenadas relativas. Isto é feito para que o momento de inércia fique da seguinte forma

$$
I=\left|z_{1}\right|^{2}+\left|z_{2}\right|^{2}
$$

Da mesma forma, como as rotações em $\mathbb{R}^{2}$ de $q_{1}, q_{2}$ e $q_{3}$ não alteram o potencial Newtoniano, a rotação das coordenadas $z_{1}$ e $z_{2}$ em $\mathbb{C}^{2}$ não muda o problema. Esta simetria pode ser aproveitada para reduzir o problema. Podemos tomar o quociente desta esfera em $\mathbb{C}^{2}$ pela ação de $S^{1}$, assim a cada círculo da esfera

$$
\left|z_{1}\right|^{2}+\left|z_{2}\right|^{2}=1
$$

associaremos um ponto sobre $S^{2}$, vide figura 3.12. Esta é a esfera de forma, vide figura 3.13. Isto é feito usando a aplicação de Hopf, ou aplicação de Kustaanheimo-Stiefel,

$$
\begin{gathered}
\mathfrak{K}: \mathbb{C}^{2} \rightarrow \mathbb{R} \times \mathbb{C} \equiv \mathbb{R}^{3} \\
\mathfrak{K}\left(z_{1}, z_{2}\right)=\left(u_{1}, u_{2}+i u_{3}\right)=\left(\left|z_{1}\right|^{2}-\left|z_{2}\right|^{2}, 2 \overline{z_{1}} z_{2}\right) .
\end{gathered}
$$

Note que

$$
\left|\mathfrak{K}\left(z_{1}, z_{2}\right)\right|^{2}=\left(u_{1}^{2}+u_{2}^{2}+u_{3}^{2}\right)=\left(\left|z_{1}\right|^{2}+\left|z_{2}\right|^{2}\right)^{2}=I^{2}
$$

Assim temos,

$$
(\mathfrak{K} \circ \mathfrak{J})_{I=1}=\text { Esfera de Forma. }
$$

Com esta formulação fica mais fácil calcular as coordenadas dos pontos sobre a esfera de forma a partir das configurações em $X$. Seguem alguns pontos, onde por abuso de linguagem fazemos 


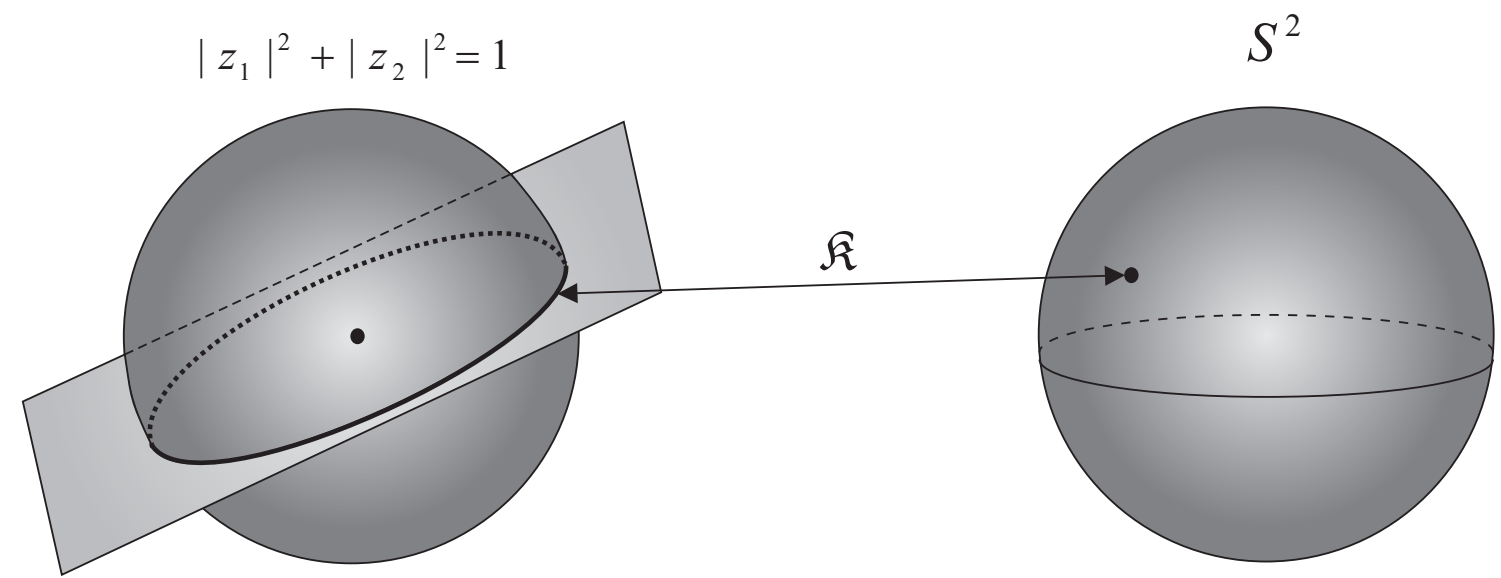

Figura 3.13: Representação da aplicação de Hopf, note que a esfera à esquerda está em $\mathbb{C}^{2}$.

$\mathfrak{K} \circ \mathfrak{J}(y)=y$,

$$
\begin{gathered}
C_{1}=(-1,0,0), \quad C_{2}=\left(\frac{1}{2}, \frac{\sqrt{3}}{2}, 0\right), \quad C_{3}=\left(\frac{1}{2},-\frac{\sqrt{3}}{2}, 0\right), \\
E_{1}=(1,0,0), \quad E_{2}=\left(-\frac{1}{2},-\frac{\sqrt{3}}{2}, 0\right), \quad E_{2}=\left(-\frac{1}{2}, \frac{\sqrt{3}}{2}, 0\right), \\
L A G_{+}=(0,0,1), \quad L A G_{-}=(0,0,-1) .
\end{gathered}
$$

Um resultado interessante é o seguinte lema de Hsiang, vide [23]

Lema 3.2.3 Para cada ponto $u=\left(u_{1}, u_{2}, u_{3}\right)$ sobre a esfera de forma existe um triângulo correspondente, cujos lados são

$$
\sqrt{a_{i}}=\sqrt{1-u \bullet C_{i}}
$$

onde convencionamos $a_{i}=$ lado oposto ao corpo $i$.

\subsubsection{A Construção da Órbita em Forma de "Oito".}

Vejamos agora como seria a representação da órbita em forma de oito sobre o espaço de triângulos orientados. Primeiro consideremos os seguintes fatos:

- Os cálculos numéricos indicavam que a órbita em oito passava pelas três configurações de Euler e por três triângulos isósceles [22];

- Outros cálculos numéricos indicavam que a órbita em oito é estável, [23]. 


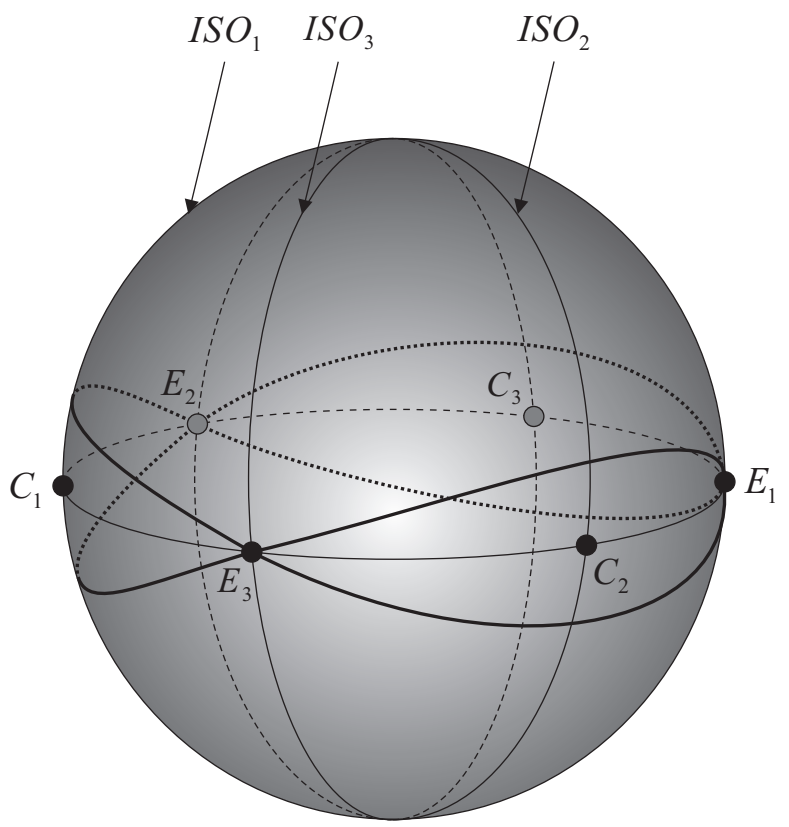

Figura 3.14: A curva do Oito sobre a esfera de forma.

Observação 3.2.4 Para visualização, comecemos de uma das configurações de Euler digamos, $E_{3}$, e imaginemos que os corpos têm condições iniciais apropriadas, de forma que daí sejam levados em algum triângulo em $I S O_{1}$.

Façamos uma reflexão desta curva no plano que passa por $E_{1}, C_{1}$ e $L A G_{+}$, isto é, o plano que contém ISO $O_{1}$. Desta forma obtemos uma curva que leva de $I S O_{1}$ em $E_{2}$. Agora vamos refletir este no plano que contém $\mathrm{ISO}_{2}$ e em seguida refletir no plano que contém o equador desta esfera, assim obteremos a curva que leva $\mathrm{E}_{2}$ em $\mathrm{ISO}_{3}$.

Prosseguindo com este procedimento, por mais nove vezes alternando os índices obteremos uma curva fechada, vide figura 3.14. Esta curva representa a órbita em forma de oito sobre a esfera de forma, olhando este esquema podemos intuir da figura que não temos colisão nestas curvas, pois em nenhum momento passamos por algum $C_{i}$.

Vejamos como seriam no Oito os instantes descritos nos parágrafos acima, primeiramente observemos na figura 3.15 a configuração $E_{3}$ sendo levada em $I S O_{1}$ e em seguida de $I S O_{1}$ para $E_{2}$. 


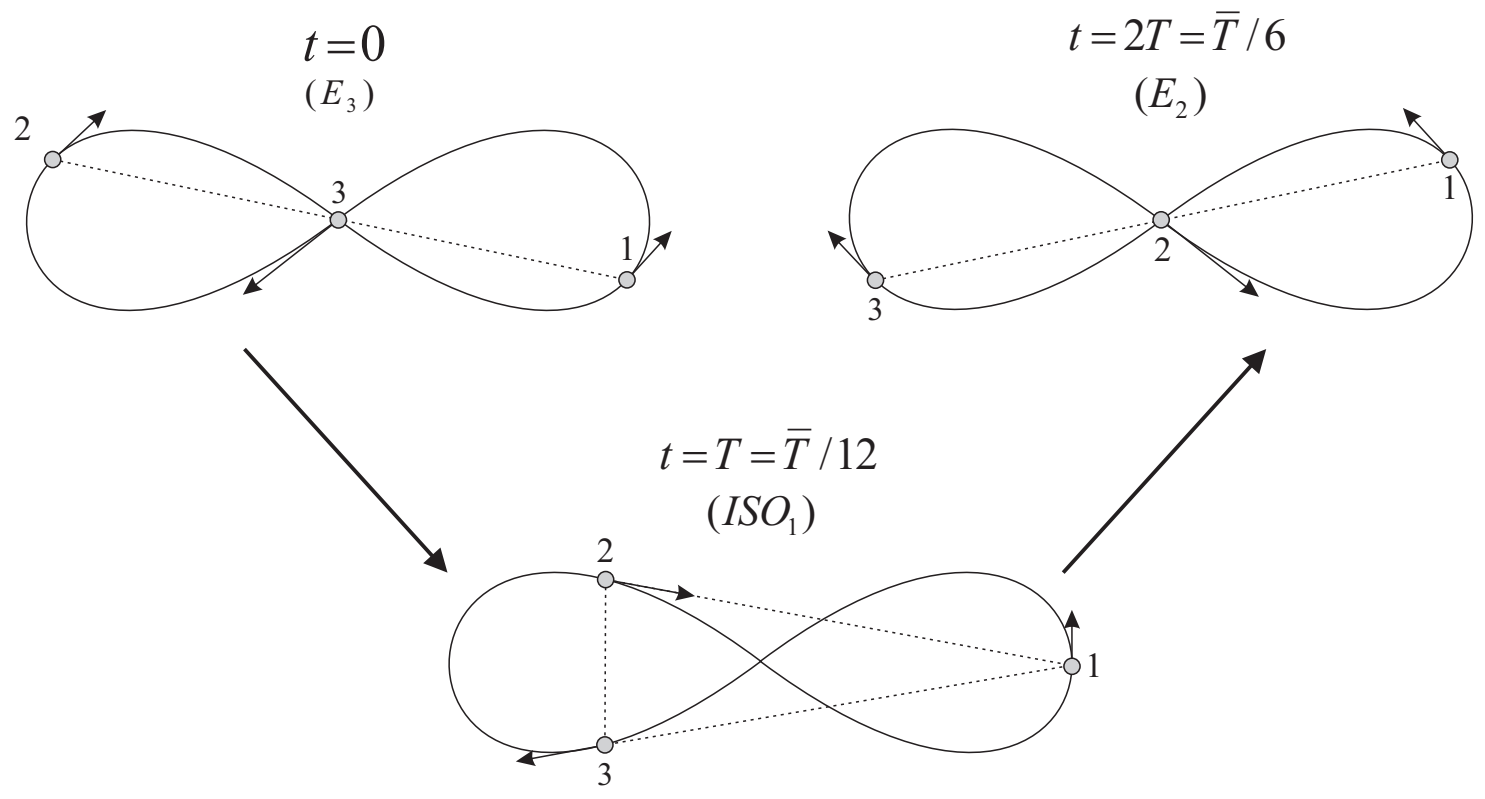

Figura 3.15: Movimento dos corpos sobre o Oito.

Na figura acima também colocamos $\bar{T}$ que representa o período total da órbita, que corresponde a doze vezes o tempo $T$, que é gasto para levar de $E_{3}$ em $I S O_{1}$. Note também que no instante inicial temos o momento angular total nulo, bem como em todas as outras configurações.

\subsubsection{A Órbita em Oito}

Seja $\bar{T} \in \mathbb{R}^{+}$, usaremos a seguinte notação para o quociente dos reais para com os intervalos de tamanho $\bar{T}$

$$
\mathbb{R} / \bar{T} \mathbb{Z} \equiv\left\{\begin{array}{l}
x, \text { se }|x|<\bar{T} \\
x-\operatorname{MIM}(\bar{T}, x), \text { se }|x| \geq \bar{T}
\end{array}\right.
$$

onde $\operatorname{MIM}(\bar{T}, x)$ indica o maior múltiplo inteiro de $\bar{T}$ menor que $x$ em módulo.

Definição 3.2.5 O grupo de Klein $\mathbb{Z}_{2} \times \mathbb{Z}_{2}$ é um grupo cujos elementos são a identidade, a reflexão vertical, a reflexão horizontal e a rotação de $\pi$. Note que o grupo de Klein assim definido tem como geradores a reflexão vertical $\sigma_{1}$ e a reflexão horizontal $\sigma_{2}$. 
Definição 3.2.6 Definimos a ação do grupo de Klein sobre $\mathbb{R} / \bar{T} \mathbb{Z}$ como sendo

$$
\begin{gathered}
\sigma_{1}(t)=t+\frac{\bar{T}}{2}, \\
\sigma_{2}(t)=-t+\frac{\bar{T}}{2}
\end{gathered}
$$

Definição 3.2.7 Definimos a ação do grupo de Klein sobre $\mathbb{R}^{2}$ como sendo

$$
\begin{aligned}
& \sigma_{1}(u, v)=(-u, v), \\
& \sigma_{2}(u, v)=(u,-v) .
\end{aligned}
$$

Colocamos abaixo o principal resultado desta seção, cuja prova será esboçada a seguir.

Teorema 3.2.8 Considere um sistema formado por três massas unitárias que realizam um movimento planar, cujas posições podem ser definidas sobre X. Então, com condições iniciais apropriadas, temos uma órbita planar em forma de Oito $Q: \mathbb{R} / \bar{T} \mathbb{Z} \rightarrow \mathbb{R}^{2}$, a qual respeita as seguintes condições

1. Para cada tempo $t$

$$
Q(t)+Q\left(t+\frac{\bar{T}}{3}\right)+Q\left(t+\frac{2 \bar{T}}{3}\right)=0
$$

2. $Q: \mathbb{R} / T \mathbb{Z} \rightarrow \mathbb{R}^{2}$ é equivariante com respeito a ação do grupo de Klein $\mathbb{Z}_{2} \times \mathbb{Z}_{2}$ sobre $\mathbb{R} / \bar{T} \mathbb{Z}$ e sobre $\mathbb{R}^{2}$, ou seja,

$$
Q\left(\sigma_{1}(t)\right)=\sigma_{1}(Q(t))
$$

$e$

$$
Q\left(\sigma_{2}(t)\right)=\sigma_{2}(Q(t))
$$

3. A órbita $q: \mathbb{R} / \bar{T} \mathbb{Z} \rightarrow X$ fica dada por

$$
q(t)=\left(Q\left(t+\frac{2 \bar{T}}{3}\right), Q\left(t+\frac{\bar{T}}{3}\right), Q(t)\right),
$$


que é uma órbita periódica de período $\bar{T}$, tal que a cada instante o momento angular total do sistema é nulo.

Antes de fazer a prova, consideremos alguns resultados que serão usados nesta. Aqui vem a importância de definirmos a órbita sobre a esfera de forma. Como mostrado acima, podemos imaginar a órbita como vários arcos obtidos uns dos outros por reflexões. Assim basta que provemos que existe uma curva que leva de $E_{3}$ até $I S O_{1}$ que respeite as condições do Oito. Vamos definir o seguinte conjunto

$$
\Lambda \equiv\left\{\text { Curvas de } X \text { que levam de } E_{3} \text { até } I S O_{1} \text { em um tempo } T\right\}
$$

onde $T=\bar{T} / 12$.

A partir deste conjunto podemos construir a curva em Oito, da seguinte maneira. Procuremos em $\Lambda$ a curva que minimiza o funcional

$$
\mathcal{S}=\int_{0}^{T} L(q, \dot{q}) d t
$$

onde $L$ é a função principal de Lagrange do sistema, como definida em 3.11. A existência de tal caminho é consequência de resultados de cálculo das variações, que não serão aqui detalhados. Partindo da existência devemos apenas mostrar que neste caminho não temos colisões.

Proposição 3.2.9 Um caminho de $\Lambda$ que minimiza a ação 3.17 não tem colisões.

Prova: Vamos fazer uso de dois lemas, seguindo o trabalho de Kuo-Chang Chen em [26]. Definamos $\Lambda_{c}$ o conjunto das curvas de $\Lambda$ que passam por alguma colisão, que é

$$
\Lambda_{c} \equiv\left\{\text { Curvas de } \Lambda \text {, tais que } q_{i}(s)=q_{j}(s) \text {, para algum } i \neq j \text { e } s \in[0, T]\right\}
$$

Assim queremos mostrar que vale a seguinte

$$
\inf _{q \in \Lambda} \mathcal{S}(q)<\inf _{q \in \Lambda_{c}} \mathcal{S}(q)
$$


Em verdade, o lado esquerdo é aproximadamente $2.53 T^{1 / 3}$ e o lado direito tem cota inferior aproximadamente igual a $2.55 T^{1 / 3}$, vide $[26]$.

Lema 3.2.10 Assuma que $T=1$. Então, $\mathcal{S}(q)>2.87$ para qualquer $q \in \Lambda_{c}$.

Demonstração: Fixemos um $q \in \Lambda_{c}$ e definamos,

$$
\mu=\mu_{q} \doteq \max _{s_{1}, s_{2} \in[0, T]}\left|q\left(s_{1}\right)-q\left(s_{2}\right)\right|
$$

Caso 1: $2.22 \leq \mu$. No instante inicial digamos que distâncias entre 1 e 3 seja $d$ e por conseguinte a distância de 1 a 2 é $2 d$ e que $i$ e $j$ colidem no instante $s \in[0,1]$. Assim vale a seguinte desigualdade

$$
\mu^{2} \geq\left|q_{i}(s)-q_{i}(0)\right|^{2}+\left|q_{j}(s)-q_{j}(0)\right|^{2} \geq \frac{d^{2}}{2}
$$

A primeira desigualdade é satisfeita para todo $t \in[0,1]$, daí fazendo $r_{i j}(t)=\left|q_{i}(t)-q_{j}(t)\right|$ temos

$$
\begin{aligned}
r_{13} & \leq\left|q_{1}(t)-q_{1}(0)\right|+\left|q_{1}(0)-q_{3}(0)\right|+\left|q_{3}(0)-q_{3}(t)\right| \\
& \leq \sqrt{2\left(\left|q_{1}(t)-q_{1}(0)\right|^{2}+\left|q_{3}(0)-q_{3}(t)\right|^{2}\right)}+d \\
& \leq 2 \sqrt{2} \mu
\end{aligned}
$$

De modo análogo,

$$
r_{23} \leq 2 \sqrt{2} \mu
$$

e

$$
r_{12} \leq 3 \sqrt{2} \mu
$$

Escrevendo a ação explicitamente, usando estas desigualdades acima e a de Cauchy-Schwartz, temos

$$
\begin{aligned}
\mathcal{S}(q) & =\frac{1}{2} \int_{0}^{1}|\dot{q}|^{2} d t+\int_{0}^{1}\left[\frac{1}{r_{12}}+\frac{1}{r_{13}}+\frac{1}{r_{23}}\right] d t \\
& \geq \frac{1}{2}\left(\int_{0}^{1}|\dot{q}| d t\right)^{2}+\frac{4}{3 \sqrt{2} \mu}
\end{aligned}
$$




$$
\geq \frac{\mu^{2}}{2}+\frac{2 \sqrt{2}}{3 \mu}=f(\mu)
$$

Notemos agora que a função $f(\mu)$ da última expressão é estritamente crescente para

$$
\mu \geq \frac{\sqrt{2}}{\sqrt[3]{3}}
$$

e por conseguinte o mínimo desta no intervalo $[2.22,+\infty)$ ocorre em $\mu=2.22$. Mas neste ponto $f(2.22) \approx 2.89$ que é maior que 2.87 .

Caso 2: $0 \leq \mu<2.22$. Vamos assumir, por enquanto, que a colisão ocorra entre 2 e 3 . Os outros casos podem ser feitos de modo semelhante.

Definamos

$$
w=\left(\int_{0}^{1}\left|\dot{q}_{1}\right|^{2} d t\right)^{1 / 2}
$$

então para todo $s \in[0,1]$ vale

$$
\left|q_{1}(s)-q_{1}(0)\right| \leq \int_{0}^{1}\left|\dot{q}_{1}\right| d t \leq w
$$

e

$$
\begin{aligned}
\mu^{2} & \geq\left|q_{1}(s)-q_{1}(0)\right|^{2}+\left|q_{2}(s)-q_{2}(0)\right|^{2}+\left|q_{3}(s)-q_{3}(0)\right|^{2} \\
& \geq \frac{1}{2}\left(\left|q_{1}(s)-q_{1}(0)\right|+\left|q_{2}(s)-q_{2}(0)\right|\right)^{2}+\left|q_{3}(s)-q_{3}(0)\right|^{2} \\
& \geq \frac{1}{2}\left|q_{1}(s)-q_{1}(0)+q_{2}(s)-q_{2}(0)\right|^{2}+\left|q_{3}(s)-q_{3}(0)\right|^{2}
\end{aligned}
$$

como desde o início supusemos o referencial baricêntrico podemos substituir $q_{1}+q_{2}$ por $-q_{3}$. Desta feita obteremos da última expressão o seguinte

$$
\mu^{2} \geq \frac{3}{2}\left|q_{3}(s)-q_{3}(0)\right|^{2}
$$


De modo análogo, obtemos para os outros dois corpos

$$
\mu^{2} \geq \frac{3}{2}\left|q_{1}(s)-q_{1}(0)\right|^{2}
$$

e

$$
\mu^{2} \geq \frac{3}{2}\left|q_{2}(s)-q_{2}(0)\right|^{2}
$$

Vamos agora estimar cotas superiores para $r_{12}$ e $r_{13}$ em qualquer instante, para depois fazermos a substituição na expressão da ação.

$$
\begin{aligned}
r_{13}(t) & \leq\left|q_{1}(t)-q_{1}(0)\right|+\left|q_{1}(0)-q_{3}(0)\right|+\left|q_{3}(0)-q_{3}(t)\right| \\
& \leq w+d+\sqrt{\frac{2}{3}} \mu \\
& \leq w+\sqrt{2}\left(1+\frac{1}{\sqrt{3}}\right) \mu
\end{aligned}
$$

e similarmente

$$
r_{12}(t) \leq w+2 d+\sqrt{\frac{2}{3}} \mu \leq w+\sqrt{2}\left(2+\frac{1}{\sqrt{3}}\right) \mu .
$$

Escrevamos a expressão da ação com o seguinte rearranjo nos termos sob o sinal de integração

$$
\mathcal{S}(q)=\int_{0}^{1}\left(\frac{\left|\dot{q}_{1}\right|^{2}}{2}+\frac{1}{r_{12}}+\frac{1}{r_{13}}\right) d t+\int_{0}^{1}\left[\frac{1}{2}\left(\left|\dot{q}_{2}\right|^{2}+\left|\dot{q}_{3}\right|^{2}\right)+\frac{1}{r_{23}}\right] d t
$$

Notemos que o segundo termo é a ação de um problema de dois corpos, que no caso de órbitas do tipo colisão-ejeção teve sua cota inferior calculada por W. Gordon, em [27], e por ele chamada de $\mathcal{A}^{2}$. Usando o resultado de Gordon temos

$$
\mathcal{A}_{2}=\frac{3}{2}\left(\frac{\pi^{2}}{2}\right)^{1 / 3}
$$

Voltando à expressão da ação, nos vem

$$
\mathcal{S}(q) \geq \int_{0}^{1}\left(\frac{\left|\dot{q}_{1}\right|^{2}}{2}+\frac{1}{r_{12}}+\frac{1}{r_{13}}\right) d t+\mathcal{A}_{2}
$$




$$
\begin{aligned}
& \geq \frac{w^{2}}{2}+\frac{1}{w+\sqrt{2}(2+\sqrt{3} / 3) \mu}+\frac{1}{w+\sqrt{2}(1+\sqrt{3} / 3) \mu} \\
& >\frac{w^{2}}{2}+\frac{1}{w+\sqrt{2}(2+\sqrt{3} / 3) 2.22}+\frac{1}{w+\sqrt{2}(1+\sqrt{3} / 3) 2.22}=g(w) .
\end{aligned}
$$

A função $g(w)$ tem um mínimo aproximadamente em $w \approx 0.05495356$ e seu valor é aproximadamente $g(0.05495356) \approx 0.32397448>0.32397$. Juntando estas estimativas temos

$$
\mathcal{S}(q)>\mathcal{A}_{2}+0.32397=2.55376+0.32397=2.87773>2.87 .
$$

Com isto provamos o lema.

Para nosso caso vamos usar o seguinte corolário do lema 3.2.10.

Corolário 3.2.11 Considerando o mesmo problema do lema com um $T$ qualquer, teremos que vale

$$
\mathcal{S}(q)>2.87 T^{1 / 3}
$$

para qualquer $q \in \Lambda_{c}$.

Por fim devemos usar o seguinte lema

Lema 3.2.12 Existe um caminho $q \in \Lambda$ com ação $\mathcal{S}(q)<2.64 T^{1 / 3}$.

Demonstração: Vamos tomar uma parametrização da esfera de forma como segue

$$
\left(u_{1}, u_{2}, u_{3}\right)=(\cos \theta \cos \phi, \cos \theta \sin \phi, \sin \theta)
$$

usando o lema 3.2.3 podemos escrever o potencial Newtoniano, restrito a $I=1$, nestas coordenadas da seguinte maneira

$$
-V(\phi, \theta)=\frac{1}{\sqrt{1+\cos \theta \cos \phi}}+\frac{1}{\sqrt{1+\cos \theta \cos (\phi+2 \pi / 3)}}+\frac{1}{\sqrt{1+\cos \theta \cos (\phi+4 \pi / 3)}}
$$


Vamos agora restringir nossa atenção ao potencial sobre o círculo da esfera de forma que respeita a seguinte condição

$$
\theta=\arctan (2 \sin \phi)
$$

donde recebemos

$$
-V(\phi)=\frac{1}{\sqrt{1+\frac{\cos \phi}{\sqrt{1+4 \sin ^{2} \phi}}}}+\frac{1}{\sqrt{1+\frac{\cos \phi+2 \pi / 3}{\sqrt{1+4 \sin ^{2} \phi}}}}+\frac{1}{\sqrt{1+\frac{\cos \phi+4 \pi / 3}{\sqrt{1+4 \sin ^{2} \phi}}}}
$$

Podemos ver que $-V(\phi)$, com $\phi \in[0, \pi / 3]$, tem valor máximo de aproximadamente $\nu=3.535734<$ 3.536. Seja agora $\mathcal{Q} \in \Lambda$ o caminho com $I$ qualquer que leva de $E_{3}$ em $I S O_{1}$ com velocidade constante e $\eta$ o comprimento deste caminho sobre a esfera. Podemos verificar que

$$
\eta=\frac{1}{2} \arctan \left(\frac{1}{4}\right) \approx 0.659058<0.66
$$

Desta forma a ação de $\mathcal{Q}$ deve satisfazer a seguinte desigualdade

$$
\mathcal{S}(\mathcal{Q})=\int_{0}^{T}\left[\frac{1}{2}\left(\frac{\eta \sqrt{I}}{T}\right)-\frac{1}{\sqrt{I}} V(\phi(t))\right] d t<\frac{1}{2}\left(\frac{\eta^{2} I}{T}\right)+\frac{\nu T}{\sqrt{I}}=h(I)
$$

Derivando $h$ em relação a $I$ vemos que ela assume valor mínimo em

$$
I=\left(\frac{\nu T^{2}}{\eta^{2}}\right)^{2 / 3}
$$

Levando agora este $I$ na expressão da ação de $\mathcal{Q}$ temos

$$
\begin{aligned}
\mathcal{S}(\mathcal{Q}) & <\frac{1}{2}\left(\frac{\eta^{2}}{T}\right)\left(\frac{\nu T^{2}}{\eta^{2}}\right)^{2 / 3}+\nu T\left(\frac{\nu T^{2}}{\eta^{2}}\right)^{-1 / 3} \\
& =\frac{3}{2}(\nu \eta)^{2 / 3} T^{1 / 3} \approx 2.636494 T^{1 / 3} \\
& <2.64 T^{1 / 3}
\end{aligned}
$$

Com isto provamos o lema. 
Juntando o resultado do corolário 3.2.11 e do lema 3.2.12 temos o resultado da proposição. Com isto provamos o lema.

Assim podemos dizer que o caminho que leva de $E_{3}$ em $I S O_{1}$ e minimiza o funcional ação não tem colisões. Por conseguinte a primeira parte do Oito está bem definida.

Para fazermos a prova do teorema principal resta-nos mostrar então que solução obtida como nos comentários da observação 3.2.4 respeita às condições do teorema.

\section{Prova do teorema 3.2.8:}

Seja $Q: \mathbb{R} / \bar{T} \mathbb{Z} \rightarrow \mathbb{R}^{2}$ a curva que descreve o movimento de um corpo sobre o oito, vamos mostrar que neste caso temos satisfeitas as condições do teorema principal. Consideremos então que

$$
Q(t) \doteq q_{3}(t)
$$

Cada arco ligando uma configuração de Euler à configuração isósceles mais próxima é percorrido num tempo $T=\bar{T} / 12$, pela construção da esfera de forma. Consideremos então o seguinte esquema para as posições mostrados nas figuras abaixo.

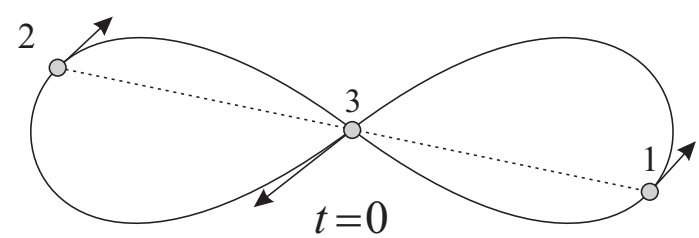

Figura 3.16: Instante $t=0$.

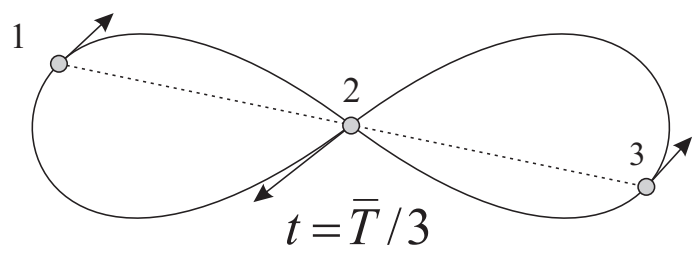

Figura 3.17: Instante $t=\bar{T} / 3$.

Note que nas figuras acima, 3.17 corresponde a 3.16 após feita uma permutação cíclica dos corpos, isto é, de 3.18 decorre que

$$
q_{2}(t)=Q(t+\bar{T} / 3)
$$

De modo análogo, note que nas figuras abaixo 3.19 corresponde a 3.18 após feita duas permutações 


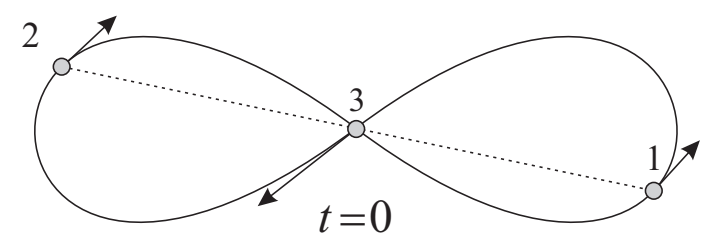

Figura 3.18: Instante $t=0$.

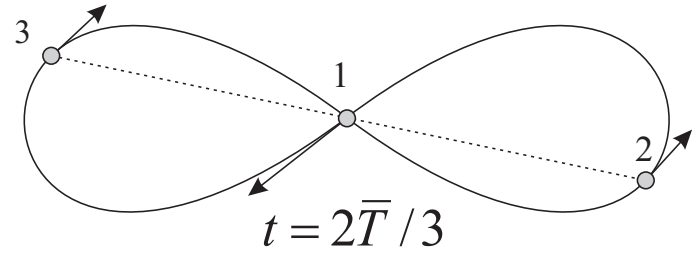

Figura 3.19: Instante $t=\bar{T} / 3$.

cíclicas dos corpos, isto é, de 3.18 decorre que

$$
q_{1}(t)=Q(t+2 \bar{T} / 3)
$$

De 3.18, 3.19 e 3.20 temos a seguinte expressão para a órbita no espaço $X$

$$
q(t)=\left(Q\left(t+\frac{2 \bar{T}}{3}\right), Q\left(t+\frac{\bar{T}}{3}\right), Q(t)\right)
$$

deste fato e considerando que estamos no referencial do centro de massa, temos

$$
Q\left(t+\frac{2 \bar{T}}{3}\right)+Q\left(t+\frac{\bar{T}}{3}\right)+Q(t)=0
$$

que é o item 1 do teorema.

O item 2 do teorema decorre diretamente da construção feita acima para $Q$ e do lema 3.2.3.

Para vermos o item 3 do teorema, dentre outros possíveis caminhos, podemos usar a decomposição de velocidades de D.Saari, que pode ser encontrada em [28]. No caso do problema planar de três corpos esta decomposição nas coordenadas esféricas do espaço de triângulos orientados, fica dada por

$$
T=\dot{R}^{2}+\frac{R^{2}}{4}\left(\dot{\theta}^{2}+\dot{\phi}^{2} \cos ^{2} \theta\right)+\frac{J^{2}}{R^{2}}+\frac{|P|^{2}}{M}
$$

onde $R^{2}=I$ representa o momento de inércia da configuração, $J$ o módulo do momento angular total, $P$ é o momento linear total do sistema e $M$ a massa total do sistema. Vide [29]. 
No problema considerado aqui $P=0$, pois estamos no referencial baricêntrico. Desta feita a energia cinética fica dada por

$$
T=\dot{R}^{2}+\frac{R^{2}}{4}\left(\dot{\theta}^{2}+\dot{\phi}^{2} \cos ^{2} \theta\right)+\frac{J^{2}}{R^{2}}
$$

Assim escrevamos a ação com essa decomposição,

$$
\mathcal{S}=\int_{0}^{T}\left[\dot{R}^{2}+\frac{R^{2}}{4}\left(\dot{\theta}^{2}+\dot{\phi}^{2} \cos ^{2} \theta\right)+\frac{J^{2}}{R^{2}}-V\right] d t
$$

claramente temos a seguinte desigualdade

$\int_{0}^{T}\left[\dot{R}^{2}+\frac{R^{2}}{4}\left(\dot{\theta}^{2}+\dot{\phi}^{2} \cos ^{2} \theta\right)-V\right] d t<\int_{0}^{T}\left[\dot{R}^{2}+\frac{R^{2}}{4}\left(\dot{\theta}^{2}+\dot{\phi}^{2} \cos ^{2} \theta\right)+\frac{J^{2}}{R^{2}}-V\right] d t, \quad \forall J>0$.

Assim um caminho que minimiza a ação de $E_{1}$ e $I S O_{1}$ deve ter momento angular total nulo. Disto e da equação 3.21 temos o item 3 do teorema.

Recentemente uma outra prova para a existência desta órbita foi feita por R. Moeckel em [30]. 


\section{Capítulo 4}

\section{O Problema de $n$-Corpos}

Neste capítulo vamos fazer uma breve apresentação sobre o problema geral de $n$ massas interagindo gravitacionalmente. Este Problema surge como pergunta natural após os trabalhos de Newton.

Considere um sistema formado por $n$ partículas de massas $m_{1}, m_{2}, \ldots, m_{n}$ localizadas no espaço Euclidiano $\mathbb{R}^{3}$ pelas coordenadas $q_{1}, q_{2}, \ldots, q_{n}$, respectivamente. Suponha que a interação sobre a $i$ ésima partícula para com a j-ésima seja dada pela Lei da Gravitação Universal de Newton, conforme enunciado no capítulo 2. Suponha também que seja válido o princípio da superposição. Assim, a Lagrangiana do sistema é dada por

$$
L=\frac{1}{2} \sum_{i=1}^{n} m_{i} \dot{q}_{i}^{2}+\sum_{1 \leq i<j \leq n} \frac{G m_{i} m_{j}}{\left|q_{i}-q_{j}\right|}
$$

Em uma notação compactada, podemos escrever a equação de Euler-Lagrange para 4.1 na seguinte forma

$$
M \ddot{Q}=-\nabla V
$$

onde usamos uma matriz diagonal

$$
M=\operatorname{diag}\left[m_{1}, m_{2}, \ldots, m_{n}\right]
$$


um vetor de $\mathbb{R}^{3 n}$

$$
Q=\left(q_{1}, q_{2}, \ldots, q_{n}\right)
$$

um operador diferencial

$$
\nabla=\left(\nabla_{1}, \nabla_{2}, \ldots, \nabla_{n}\right)
$$

com $\nabla_{k}$ sendo o gradiente nas coordenadas do $k$-ésimo corpo e a energia potencial

$$
V=-\sum_{1 \leq i<j \leq n} \frac{G m_{i} m_{j}}{\left|q_{i}-q_{j}\right|}
$$

É fácil notar que expressão 4.3 não está bem definida quando temos sobreposição de corpos. Vamos restringir nossa atenção fora deste caso.

Definição 4.0.13 Chamamos de conjunto colisão o seguinte

$$
\triangle=\bigcup_{i \neq j} \triangle_{i j}
$$

onde

$$
\triangle_{i j}=\left\{Q=\left(q_{1}, \ldots, q_{n}\right) \in \mathbb{R}^{3 n} / q_{i}=q_{j}\right\}
$$

Assim vamos restringir nossa atenção ao caso em que $V:\left\{\mathbb{R}^{3 n} \backslash \triangle\right\} \rightarrow(-\infty, 0)$ enquanto estudarmos este problema. Desta feita tomaremos condições iniciais

$$
Q(0) \in\left\{\mathbb{R}^{3 n} \backslash \triangle\right\},
$$

e

$$
\dot{Q}(0) \in \mathbb{R}^{3 n} .
$$




\subsection{Não-Integrabilidade do Problema de $n$-corpos}

Podemos pensar no problema de $n$-corpos como um problema de valor inicial

$$
\left\{\begin{array}{l}
\dot{S}=F(S) \equiv\left(\dot{Q},-M^{-1} \nabla V\right) \\
S(0)=(Q(0), \dot{Q}(0)) \in\left\{\mathbb{R}^{3 n} \backslash \triangle\right\} \times \mathbb{R}^{3 n}
\end{array},\right.
$$

onde $S=(Q, \dot{Q}) \in \mathbb{R}^{6 n}$ e $F: \mathbb{R}^{3 n} \times\left\{\mathbb{R}^{3 n} \backslash \triangle\right\} \rightarrow \mathbb{R}^{6 n}$ é continuamente diferenciável, pelo menos num aberto em torno de $(Q(0), \dot{Q}(0)) \in \mathbb{R}^{6 n}$. Tal fato advém da escolha das posições iniciais fora de $\triangle$. Isto é suficiente para usarmos o teorema de existência e unicidade, vide [31], para garantir que existe $\delta \in \mathbb{R}$ e uma única aplicação

$$
\begin{aligned}
& S:(-\delta, \delta) \rightarrow \mathbb{R}^{6 n} \\
& t \quad \mapsto S(t)=(Q(t), \dot{Q}(t))
\end{aligned}
$$

solução de 4.4. Muito embora tal solução exista e seja única ela não pode ser determinada por quadraturas, pois de acordo com o próximo resultado, de E. Julliard-Toseal em [32], temos apenas 10 integrais primeiras para o problema de $n$-corpos em $\mathbb{R}^{3}$. Em verdade, Julliard-Tosel generalizou os resultados obtidos por H. Bruns em [2]. Abaixo enunciamos o teorema de Bruns generalizado.

Teorema 4.1.1 No problema Newtoniano de $(n+1)$-corpos em $\mathbb{R}^{p}$, com $n \geq 2$ e $1 \leq p \leq n+1$, toda integral primeira que seja algébrica com respeito às posiçôes, momentos e tempo é uma função algébrica das integrais primeiras clássicas: a energia, as $p(p-1) / 2$ componentes do momento angular total e as $2 p$ integrais que vem do movimento retilíneo uniforme do centro de massa.

Para nosso caso temos $p=3$, e as integrais primeiras seguem. Primeiramente a energia fica dada por

$$
E=T+V=\sum_{i=1}^{n} m_{i} \dot{q}_{i}^{2}-\sum_{1 \leq i<j \leq n} \frac{G m_{i} m_{j}}{\left|q_{i}-q_{j}\right|},
$$


em seguida o momento angular total, o qual seja

$$
J=\sum_{i=1}^{n} m_{i} q_{i} \wedge \dot{q}_{i}
$$

e por fim, o momento linear total

$$
B=\sum_{i=1}^{n} m_{i} \dot{q}_{i} .
$$

Contando quantas quantidades escalares temos em 4.5-4.7, vemos que o resultado é exatamente 10 quantidades. Agora se observarmos a expressão 4.2 vemos que esta é uma equação diferencial em $\mathbb{R}^{6 n}$ e pelo teorema anterior se $n \geq 2$ não conhecemos métodos para determinar diretamente sua solução. O que é feito geralmente é estudas casos particulares, fazendo restrições nas massas ou nas próprias configurações ao longo do tempo. No próximo capítulo vamos estudar um tipo de solução possível, que é o caso em que as posições iniciais formam configurações centrais.

\subsection{Alguns resultados do problema geral de $n$-corpos}

Vamos introduzir aqui alguns conceitos e teoremas que foram obtidos ao longo dos mais de trezentos ano de estudo do problema de $n$-corpos.

Definição 4.2.1 Chamaremos de momento de inércia generalizado a seguinte quantidade escalar

$$
\zeta=\frac{1}{2} \sum_{i=1}^{n} m_{i}\left|q_{i}\right|^{2}
$$

que também pode ser escrita como

$$
\zeta=\frac{1}{2 m_{t}} \sum_{1 \leq i<j \leq n}^{n} m_{i} m_{j}\left|q_{i}-q_{j}\right|^{2}
$$

onde $m_{t}=\sum_{i=1}^{n} m_{i}$ é massa total do sistema.

Teorema 4.2.2 No problema de n-corpos, conforme descrito acima, vale a seguinte igualdade

$$
\ddot{\zeta}=2 T+V=T+E=2 E-V .
$$


Esta é a chamada de identidade Lagrange-Jacobi.

Prova: Derivando a expressão 4.8 obtemos,

$$
\dot{\zeta}=\frac{1}{2} \sum_{i=1}^{n} m_{i} q_{i} \bullet \dot{q}_{i}
$$

e por conseguinte,

$$
\ddot{\zeta}=\sum_{i=1}^{n} m_{i} \dot{q}_{i}^{2}+\sum_{i=1}^{n} m_{i} q_{i} \bullet \ddot{q}_{i} .
$$

Lembrando que V é uma função homogênea de grau (-1) nas componentes dos $q_{i}$ 's, temos

$$
\sum_{i=1}^{n} q_{i} \bullet \nabla_{i} V=-V
$$

$\log$,

$$
\ddot{\zeta}=2 T+\sum_{i=1}^{n} q_{i} \bullet \nabla_{i} V=2 T+V=T+E=2 E-V .
$$

Teorema 4.2.3 Para o problema de n-corpos, descrito acima, vale a seguinte desigualdade para o módulo do momento angular

$$
J^{2} \leq 4 \zeta(\ddot{\zeta}-E)
$$

Esta é a chamada desigualdade de Sundman.

Prova: Lembremos que

$$
|J|=\left|\sum_{i=1}^{n} m_{i} q_{i} \wedge \dot{q}_{i}\right|,
$$

Logo pelas desigualdades triangular e de Cauchy-Schwartz

$$
|J| \leq \sum_{i=1}^{n}\left|m_{i} q_{i} \wedge \dot{q}_{i}\right| \leq \sum_{i=1}^{n} m_{i}\left|q_{i}\right|\left|\dot{q}_{i}\right|=\sum_{i=1}^{n} \sqrt{m_{i}}\left|q_{i}\right| \sqrt{m_{i}}\left|\dot{q}_{i}\right|
$$


que ainda podemos escrever como sendo

$$
|J| \leq \sum_{i=1}^{n} \sqrt{m_{i}}\left|q_{i}\right| \sqrt{m_{i}}\left|\dot{q}_{i}\right| \leq \sqrt{\sum_{i=1}^{n} m_{i} q_{i}^{2}} \sqrt{\sum_{i=1}^{n} m_{i} \dot{q}_{i}{ }^{2}},
$$

donde

$$
|J| \leq \sqrt{\sum_{i=1}^{n} m_{i} q_{i}^{2}} \sqrt{\sum_{i=1}^{n} m_{i} \dot{q}_{i}^{2}}=\sqrt{2 \zeta} \sqrt{2 T}=\sqrt{4 \zeta T},
$$

elevando ambos os membros ao quadrado e usando o teorema anterior temos

$$
J^{2} \leq 4 \zeta T=4 \zeta(\ddot{\zeta}-E)
$$

Teorema 4.2.4 No problema de $n$-corpos existem constantes positivas $a_{1}, a_{2}, a_{3}, a_{4}$, que dependem apenas das massas, tais que valem as seguintes

$$
a_{1} \sqrt{\zeta} \leq \max _{1 \leq i<j \leq n}\left|q_{i}-q_{j}\right| \leq a_{2} \sqrt{\zeta}
$$

$e$

$$
-\frac{a_{3}}{V} \leq \min _{1 \leq i<j \leq n}\left|q_{i}-q_{j}\right| \leq-\frac{a_{4}}{V}
$$

A seguir vamos usar estes resultados para demonstrar alguns teoremas acerca do colapso total, que é o caso em que todos os corpos colidem num mesmo instante. Uma exposição direta e bem escrita destes teoremas pode ser encontrada em [33].

Lema 4.2.5 No problema de n-corpos, se ocorrer o colapso total então ele ocorre em tempo finito.

Prova: Observemos primeiramente que no colapso total temos $\zeta=0$. Suponha, por contradição, que o colapso ocorre em $t_{c}=+\infty$. Vamos definir $d_{\text {min }}(t)$ como sendo a menor distância entre os corpos no instante $t$, que é

$$
d_{\min }(t)=\min _{i \neq j}\left|q_{i}(t)-q_{j}(t)\right|
$$


$\log \mathrm{O}$

$$
\lim _{t \rightarrow+\infty} d_{\min }(t)=0
$$

Portanto, o potencial $V$ no colapso

$$
\lim _{t \rightarrow+\infty} V(t)=-\infty
$$

Pela identidade de Lagrange-Jacobi temos,

$$
\lim _{t \rightarrow+\infty} \ddot{\zeta}(t)=2 E-\lim _{t \rightarrow+\infty} V(t)=+\infty .
$$

Assim, existe $t_{1}$ tal que,

$$
t>t_{1} \Rightarrow \ddot{\zeta}(t) \geq 1
$$

Integrando esta desigualdade duas vezes obtemos,

$$
\zeta(t) \geq \frac{1}{2} t^{2}+c_{1} t+c_{2}
$$

onde $c_{1}$ e $c_{2}$ são constantes de integração que dependem apenas de $t_{1}$. Desta forma temos,

$$
\lim _{t \rightarrow+\infty} \zeta(t)=+\infty
$$

mas isto é uma contradição, já que no colapso temos $\zeta=0$. O mesmo raciocínio pode ser usado para o caso em que o colapso se dê em $t=-\infty$.

Teorema 4.2.6 No problema de n-corpos, se ocorre o colapso total,

$$
\bar{Q}=\left\{\left(q_{1}, \ldots, q_{n}\right) \in \triangle / q_{1}=q_{2}=\ldots=q_{n}\right\}
$$

então o momento angular total é nulo. Este resultado é conhecido como teorema de SundmanWeierstrass. 
Prova: Pelo lema 4.2.5 podemos considerar que o tempo de colapso é $t_{c}$, com $t_{c}<\infty$. Daí temos

$$
\lim _{t \rightarrow t_{c}} d_{\min }(t)=0
$$

$\log 0$,

$$
\lim _{t \rightarrow t_{c}} V(Q(t))=-\infty
$$

Usando a igualdade de Lagrange-Jacobi nos vem que

$$
\lim _{t \rightarrow t_{c}} \ddot{\zeta}(Q(t))=+\infty
$$

Como $\zeta(Q)$ é regular em $Q$, podemos garantir que numa vizinhança à esquerda de $t_{c}$ vale $\ddot{\zeta}(Q(t))>$ 0 . Por definição $\zeta(Q)$ é uma função não negativa, portanto podemos concluir que $\dot{\zeta}(Q(t))<0$ nessa vizinhança. Usando agora a desigualdade de Sundman podemos escrever

$$
\ddot{\zeta}(Q(t)) \geq \frac{J^{2}}{4 \zeta(Q(t))}+E .
$$

Multiplicando ambos os membros por $-\dot{\zeta}(Q(t))>0$, temos

$$
\dot{\zeta} \ddot{\zeta} \geq \frac{J^{2} \dot{\zeta}}{4 \zeta}+E \dot{\zeta}
$$

Daí integrando temos

$$
-\frac{1}{2} \frac{d}{d t}(\dot{\zeta}) \geq-\frac{J^{2}}{4} \frac{d}{d t} \ln \zeta-E \frac{d}{d t} \zeta
$$

Integrando 4.10, agora definidamente em $\left[t_{1}, t_{2}\right]$, com $t_{2}<t_{c}$, vem

$$
-\frac{1}{2}\left[\left(\dot{\zeta}\left(t_{2}\right)\right)^{2}-\left(\dot{\zeta}\left(t_{1}\right)\right)^{2}\right] \geq-\frac{J^{2}}{4} \ln \left(\frac{\zeta\left(t_{2}\right)}{\zeta\left(t_{1}\right)}\right)-E\left[\zeta\left(t_{2}\right)-\zeta\left(t_{1}\right)\right]
$$

que pode ser reescrito como

$$
\frac{J^{2}}{4} \ln \left(\frac{\zeta\left(t_{2}\right)}{\zeta\left(t_{1}\right)}\right) \leq E\left[\zeta\left(t_{2}\right)-\zeta\left(t_{1}\right)\right]+\frac{1}{2}\left[\left(\dot{\zeta}\left(t_{1}\right)\right)^{2}-\left(\dot{\zeta}\left(t_{2}\right)\right)^{2}\right]
$$


das considerações sobre as derivadas de $\zeta$, feitas acima, temos

$$
\left\{\begin{array}{l}
\left(\dot{\zeta}\left(t_{1}\right)\right)^{2}-\left(\dot{\zeta}\left(t_{2}\right)\right)^{2} \leq\left(\dot{\zeta}\left(t_{1}\right)\right)^{2} \\
\zeta\left(t_{2}\right)-\zeta\left(t_{1}\right) \leq \zeta\left(t_{2}\right)
\end{array}\right.
$$

Inserindo 4.11 e 4.12 em 4.10 e usando esta última consideração, nos vem

$$
\frac{J^{2}}{4} \leq \frac{E \zeta\left(t_{2}\right)+\left(\dot{\zeta}\left(t_{1}\right)\right)^{2}}{\ln \left(\frac{\zeta\left(t_{1}\right)}{\zeta\left(t_{2}\right)}\right)}
$$

Tomando agora

$$
\lim _{t_{2} \rightarrow t_{c}} \frac{E \zeta\left(t_{2}\right)+\left(\dot{\zeta}\left(t_{1}\right)\right)^{2}}{\ln \left(\frac{\zeta\left(t_{1}\right)}{\zeta\left(t_{2}\right)}\right)}=0,
$$

daí temos que no caso de colisão total

$$
J^{2}=0
$$




\section{Capítulo 5}

\section{Configurações Centrais}

Depois que Newton colocou o problema de $n$-corpos, houve muitas tentativas de resolvê-lo. Duas destas foram mostradas na seção 3.1, que eram as soluções de Euler e de Lagrange, mas se repararmos nestas soluções a restrição imposta fez com que voltássemos a ter um potencial central, ou seja, a restrição tornou possível separar o problema de três corpos em três problemas de um corpo sob ação de um campo central. De certa forma estas soluções motivaram a definição de configurações centrais do problema de $n$-corpos, que seriam configurações espaciais nas quais conseguimos separar o problema em $n$ problemas de um corpo sob ação de um campo central.

\subsection{Definições e Resultados da Literatura}

Definição 5.1.1 Em um instante fixo $t_{0}$, dizemos que $n$ massas $m_{1}, \ldots, m_{n}$ localizadas no referencial do centro de massa pelos vetores $q_{1}, \ldots, q_{n} /\left(q_{1}, \ldots, q_{n}\right) \in\left\{\mathbb{R}^{3 n}-\triangle\right\}$, respectivamente, formam uma configuração central se vale

$$
\ddot{q}_{i}=\lambda q_{i}, \quad \forall i=1, \ldots, n
$$

onde $\lambda=\lambda\left(q_{1}, \ldots, q_{n}\right)$ é uma função não nula. Em outras palavras, podemos dizer que a força resultante sobre a i-ésima massa aponta na direção do centro de massa. De modo equivalente, podemos escrever a condição de configuração central da seguinte maneira

$$
\lambda q_{i}=-\sum_{k \neq i} \frac{G m_{k}\left(q_{i}-q_{j}\right)}{\left|q_{i}-q_{j}\right|^{3}} \quad \forall i=1, \ldots, n
$$


Podemos encontrar a forma de $\lambda$ da seguinte maneira, em acordo com as definições e notações do capítulo 4

$$
m_{i} \ddot{q}_{i}=\nabla_{i} V
$$

usando a equação 5.1 , temos

$$
m_{i} \ddot{q}_{i}=\nabla_{i} V=\lambda m_{i} q_{i}
$$

Se tomarmos o produto interno por $q_{i}$ em ambos os membros, temos

$$
\left(-\nabla_{i} V\right) \bullet q_{i}=\lambda m_{i} q_{i} \bullet q_{i}
$$

Somando em $i$, lembrando que $V$ é homogênea de grau $(-1)$ e a definição do momento de inércia generalizado, vide 4.2 .1 , temos

$$
V=\lambda(2 \zeta)
$$

$\log$,

$$
\lambda=\frac{V}{2 \zeta} .
$$

Se fizermos rotações rígidas em torno do centro de massas, ou homotetias, no espaço de configurações dos n-corpos iremos preservar a propriedade que define as configurações centrais. Portanto iremos pensar em configurações centrais módulo essas operações.

Classes de Equivalência: Dizemos que duas configurações centrais são equivalentes se podemos passar de uma à outra fazendo homotetias e/ou rotações. Assim daqui por diante estaremos nos referindo à classe de equivalência de uma configuração central quando encontrarmos um representante da mesma.

Existem vários exemplos conhecidos de configurações centrais. Dentre eles o problema de dois corpos e as próprias soluções de Euler e de Lagrange mostradas acima formam a cada instante uma configuração central. No decorrer da história do problema de n-corpos muitas configurações centrais foram estudadas e catalogadas.

Definição 5.1.2 Uma solução do problema de n-corpos é homográfica se a figura geométrica de- 
scrita pelos corpos na condição inicial é preservada durante o movimento. Em outras palavras, nas soluções homográficas do problema de n-corpos as configurações dos corpos mudam apenas por rotações e homotetias.

Definição 5.1.3 Chamaremos de equilíbrio relativo as soluções do problema de n-corpos nas quais as configurações dos corpos mudam apenas por rotações.

No contexto de soluções homográficas temos o seguinte resultado que é chamado teorema de Laplace por A. Wintner em [34].

Teorema 5.1.4 Se a solução do problema de n-corpos é homográfica, então os corpos formam uma configuração central a cada instante.

Este teorema torna fundamental o estudo das configurações centrais, pois ele nos diz que se forem conhecidas todas as configurações centrais, então serão conhecidas também todas as possíveis soluções homográficas. Em verdade as soluções homográficas são as únicas soluções explícitas conhecidas para o problema de $n$-corpos, antes do uso de computadores para simulações, eram as únicas soluções conhecidas. Uma boa referência para o estudo e classificação de soluções homográficas do problema de n-corpos é o trabalho de C. Vidal e G. Renildo em [35] no qual também pode ser encontrada uma prova do teorema de Laplace enunciado acima.

Um caso particular do uso de configurações centrais em astrodinâmica é o uso de soluções do tipo equilíbrio relativo como tentativa de modelagem dos anéis de Saturno, como pode ser visto nos trabalhos de D. Saari em [36] e referências do mesmo.

No caso planar do problema de $n$-corpos, podemos fazer estudos da topologia envolvida no problema com um método análogo àquele usado no problema de dois corpos acima. Em seus trabalhos sobre topologia e mecânica S. Smale em [13] provou que as configurações centrais são as imagens inversas dos valores críticos de uma aplicação que associa a cada ponto do espaço de fase sua energia e momento angular. Assim o conhecimento das configurações centrais também é importante no entendimento da topologia do problema de $n$-corpos. 
Algumas soluções possíveis tais como as de colisão total ou de ejeção nas quais os corpos se afastam mutuamente tem relação direta com configurações centrais isso é o que nos mostra os próximos dois resultados de D. Saari em [38] e C. Maccord em [39], respectivamente.

Teorema 5.1.5 Se a solução do problema de n-corpos é a de colisão total, então o sistema tende para uma configuração central.

Teorema 5.1.6 Se a solução do problema de n-corpos é homotética, então é uma configuração central a cada instante. Em particular as soluções nas quais os corpos se afastam mutuamente mesmo que não homotéticas tendem para configurações centrais.

Algumas soluções particulares simples envolvem configurações centrais também. Um exemplo está nos trabalho de F. Moulton, vide [40], qual os $n$-corpos estão sobre uma reta. De certo modo uma generalização da solução retilínea de Euler. O resultado é a contagem das possíveis configurações centrais colineares.

Teorema 5.1.7 Para qualquer escolha das massas positivas no problema de $n$-corpos planar existem exatamente n!/2 configurações centrais colineares. Que correspondem às permutações dos corpos sobre a reta.

Assim vemos que no caso de configurações centrais colineares, dadas $n$ massas temos um número finito de configurações possíveis. De certa forma isto motiva a seguinte conjectura feita por A. Wintner no caso espacial em [34] e reformulada do S. Smale no caso planar em [37]. Abaixo segue o enunciado de Smale

Conjectura 5.1.8 Dadas $n$ massas positivas $m_{1}, \ldots, m_{n}$ é finito o número de configurações centrais planares formadas por elas?

No caso de dois corpos temos apenas uma configuração. Para três corpos temos cinco casos, as três configurações de Euler e as duas de Lagrange. Recentemente M. Hampton e R. Moeckel em [42] provaram que o número de configurações centrais planares com quatro massas é finito. O resultado segue 
Teorema 5.1.9 Existe apenas um número finito de configurações centrais para o problema planar de quatro corpos com massas positivas. Em verdade o número destas configurações está entre 32 e 8472.

Abaixo colocamos a representação de duas configurações centrais planares para o problema de quatro corpos.
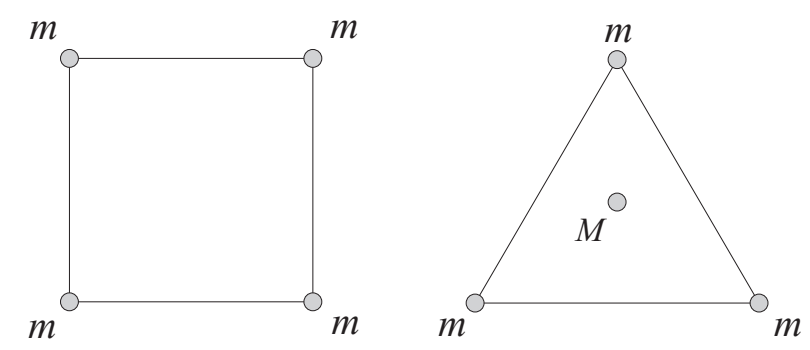

Figura 5.1: Dois exemplos de configurações centrais planares .

Existe um resultado devido a G. Roberts em [43] que é quase uma negação para essa conjectura, porém ele permite que uma das massas seja igual a -0.25 o que sai fora do enunciado da conjectura. O resultado segue

Teorema 5.1.10 No problema planar de 5-corpos se quatro massas são iguais a 1 e a quinta é igual a -0.25, então existe uma familia a um parâmetro de configurações centrais, com as quatro massas iguais nos vértices de um losango e a quinta, negativa, no centro.

Segue a representação do resultado de Roberts, vide figura 5.2.

\subsection{Resultados}

Primeiramente vamos definir agora um conjunto de equações que são muito úteis na determinação de configurações centrais. São as chamadas equações de Dziobek, uma referência para elas está nos trabalhos de Y. Hagihara em [44]. Aqui vamos apresentar as equações de Dziobek apenas para áreas e volumes em $\mathbb{R}^{3}$, mas podemos generalizar facilmente estas expressões para hipervolumes de $\mathbb{R}^{d}$ com $d>3$. 


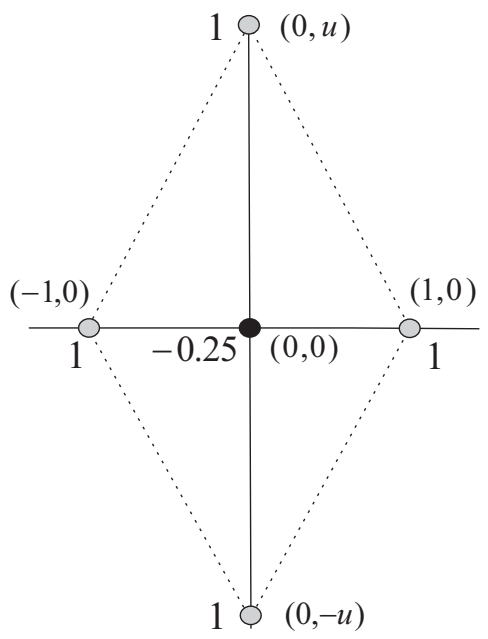

Figura 5.2: Exemplo de Roberts: temos configurações centrais para todos os valores positivos de $u$.

Definição 5.2.1 As equações de Dziobek com áreas em $\mathbb{R}^{3}$ são dadas por

$$
f_{i j}=\sum_{k \neq i, j} m_{k}\left(R_{i k}-R_{j k}\right) \Delta_{i j k}=0,1 \leq i<j \leq n
$$

onde $R_{i j}=R_{j i}=\left|q_{i}-q_{j}\right|^{3}$ e $\Delta_{i j k}=\left(q_{i}-q_{j}\right) \wedge\left(q_{i}-q_{k}\right)$ é a área do triângulo formado por $m_{i}, m_{j}$ e $m_{k}$.

Definição 5.2.2 As equações de Dziobek com volumes em $\mathbb{R}^{3}$ são dadas por

$$
f_{i j h}=\sum_{k \neq i, j, h} m_{k}\left(R_{i k}-R_{j k}\right) \Delta_{i j h k}=0,1 \leq i<j \leq n, h=1, \ldots, n
$$

onde $R_{i j}=R_{j i}=\left|q_{i}-q_{j}\right|^{3}$ e $\Delta_{i j h k}=\left(q_{i}-q_{j}\right) \wedge\left(q_{j}-q_{h}\right) \bullet\left(q_{h}-q_{k}\right)$ é o volume do tetraedro formado por $m_{i}, m_{j}, m_{h}$ e $m_{k}$.

Vamos mostrar agora que as expressões em 5.2 é equivalente a expressão 5.3.

Teorema 5.2.3 Considerando um sistema de $n$ massas não colineares, então elas formam uma configuração central se e somente se respeitam a seguinte condição

$$
f_{i j}=0, \quad \forall i, j(1 \leq i<j \leq n)
$$


Prova: Suponhamos que as $n$ massas formam uma configuração central planar. Portanto, existe $\lambda$ tal que

$$
\lambda q_{i}=-\sum_{k \neq i} m_{k} R_{i k}\left(q_{i}-q_{k}\right)
$$

Naturalmente, podemos retirar um termo da soma acima, para $j \neq i$, obtendo

$$
\lambda q_{i}=-\sum_{k \neq i, j} m_{k} R_{i k}\left(q_{i}-q_{k}\right)-m_{j} R_{i j}\left(q_{i}-q_{j}\right)
$$

Do mesmo modo para $q_{j}$ com $j \neq i$, obtemos

$$
\lambda q_{j}=-\sum_{k \neq j, i} m_{k} R_{j k}\left(q_{j}-q_{k}\right)-m_{i} R_{j i}\left(q_{j}-q_{i}\right)
$$

Considere agora a subtração de 5.5 por 5.6 , donde

$$
\lambda\left(q_{i}-q_{j}\right)=-\sum_{k \neq i, j} m_{k}\left[R_{i k}\left(q_{i}-q_{k}\right)-R_{j k}\left(q_{j}-q_{k}\right)\right]-\left[m_{j} R_{i j}-m_{i} R_{j i}\right]\left(q_{i}-q_{j}\right) .
$$

Tomando produto o vetorial por $\left(q_{i}-q_{j}\right)$ em ambos os membros de 5.7, temos

$$
0=-\sum_{k \neq i, j} m_{k}\left(R_{i k}-R_{j k}\right) \Delta_{i j k}=-f_{i j}
$$

Logo, $f_{i j}=0$, para todo $1 \leq i<j \leq n$.

Reciprocamente, consideremos que as equações de Dziobek são verificadas

$$
f_{i j}=\sum_{k \neq i, j}^{n} m_{k}\left(R_{i k}-R_{j k}\right)\left(q_{i}-q_{j}\right) \wedge\left(q_{i}-q_{k}\right)=0
$$

para $1 \leq i<j \leq n$, as quais podem ser escritas da forma

$$
\sum_{k \neq i, j}^{n} m_{k} R_{i k}\left(q_{i}-q_{j}\right) \wedge\left(q_{i}-q_{k}\right)=\sum_{k \neq i, j}^{n} m_{k} R_{j k}\left(q_{i}-q_{j}\right) \wedge\left(q_{i}-q_{k}\right) .
$$


No membro esquerdo de 5.8 podemos incluir o termo em $j$ sem alterar a igualdade. Da mesma forma, podemos inserir o termo em $i$ no membro direito. Assim, temos

$$
\sum_{k \neq i}^{n} m_{k} R_{i k}\left(q_{i}-q_{j}\right) \wedge\left(q_{i}-q_{k}\right)=\sum_{k \neq j}^{n} m_{k} R_{j k}\left(q_{i}-q_{j}\right) \wedge\left(q_{i}-q_{k}\right),
$$

ou seja,

$$
\left(q_{i}-q_{j}\right) \wedge \sum_{k \neq i}^{n} m_{k} R_{i k}\left(q_{i}-q_{k}\right)=\sum_{k \neq j}^{n} m_{k} R_{j k}\left[q_{i} \wedge\left(q_{j}-q_{k}\right)+\left(q_{j} \wedge q_{k}\right)\right] .
$$

Denotando $\nabla_{i} V$ por $F_{i}$, vemos que a igualdade 5.9 pode ser escrita como

$$
\left(q_{i}-q_{j}\right) \wedge \frac{F_{i}}{m_{i}}=\sum_{k \neq j}^{n} m_{k} R_{j k}\left[q_{i} \wedge\left(q_{j}-q_{k}\right)+\left(q_{j} \wedge q_{k}\right)\right]
$$

Podemos inserir à direita da igualdade 5.9 o termo $-q_{j}$ sem alterá-la, obtendo

$$
\left(q_{i}-q_{j}\right) \wedge \frac{F_{i}}{m_{i}}=\sum_{k \neq j}^{n} m_{k} R_{j k}\left[q_{i} \wedge\left(q_{j}-q_{k}\right)+q_{j} \wedge\left(-q_{j}+q_{k}\right)\right] .
$$

rearranjando o lado direito temos

$$
\begin{aligned}
\left(q_{i}-q_{j}\right) \wedge \frac{F_{i}}{m_{i}} & =\sum_{k \neq j}^{n} m_{k} R_{j k}\left[q_{i} \wedge\left(q_{j}-q_{k}\right)-q_{j} \wedge\left(q_{j}-q_{k}\right)\right] \\
& =\sum_{k \neq j}^{n} m_{k} R_{j k}\left[\left(q_{i}-q_{j}\right) \wedge\left(q_{j}-q_{k}\right)\right] \\
& =\left(q_{i}-q_{j}\right) \wedge \frac{F_{j}}{m_{j}}
\end{aligned}
$$

donde

$$
\left(q_{i}-q_{j}\right) \wedge \frac{F_{i}}{m_{i}}=\left(q_{i}-q_{j}\right) \wedge \frac{F_{j}}{m_{j}}
$$

a qual implica que

$$
\left(q_{i}-q_{j}\right) \wedge\left(m_{j} F_{i}-m_{i} F_{j}\right)=0 .
$$


Efetuando o produto vetorial termo a termo em 5.10 , obtemos

$$
q_{i} \wedge m_{j} F_{i}-q_{i} \wedge m_{i} F_{j}-q_{j} \wedge m_{j} F_{i}+q_{j} \wedge m_{i} F_{j}=0
$$

de onde temos

$$
m_{j} q_{i} \wedge F_{i}-m_{i} q_{i} \wedge F_{j}-m_{j} q_{j} \wedge F_{i}+m_{i} q_{j} \wedge F_{j}=0
$$

Somando em $j$ com $j \neq i$, temos

$$
\left(M-m_{i}\right) q_{i} \wedge F_{i}-m_{i} q_{i} \wedge \sum_{j \neq i}^{n} F_{j}-\left(\sum_{j \neq i}^{n} m_{j} q_{j}\right) \wedge F_{i}+m_{i} \sum_{j \neq i}^{n} q_{j} \wedge F_{j}=0
$$

onde $M$ é a massa total. Como o centro de massa está na origem do referencial, temos

$$
\sum_{j=1}^{n} m_{j} q_{j}=0 \Longrightarrow \sum_{j \neq i}^{n} m_{j} q_{j}=-m_{i} q_{i}
$$

Visto que o espaço é homogêneo e isotrópico e o sistema é isolado, temos que as quantidades de momento linear total e momento angular total são conservadas. Então, respectivamente, temos

$$
\sum_{j=1}^{n} F_{j}=0 \Longrightarrow \sum_{j \neq i}^{n} F_{j}=-F_{i}
$$

$\mathrm{e}$

$$
\sum_{j=1}^{n}\left(q_{j} \wedge F_{j}\right)=0 \Longrightarrow \sum_{j \neq i}^{n}\left(q_{j} \wedge F_{j}\right)=\left(-q_{i} \wedge F_{i}\right) .
$$

Substituindo 5.12, 5.13 e 5.14 em 5.11, obtemos

$$
M q_{i} \wedge F_{i}-m_{i} q_{i} \wedge F_{i}+m_{i} q_{i} \wedge F_{i}+m_{i} q_{i} \wedge F_{i}-m_{i} q_{i} \wedge F_{i}=0
$$

Desta forma, $M q_{i} \wedge F_{i}=0$, logo $q_{i}$ e $F_{i}$ são paralelos, ou seja, $F_{i}=\lambda_{i} q_{i}$, ou ainda $\ddot{q}_{i}=\left(\lambda_{i} / m_{i}\right) q_{i}$. De 5.10, decorre

$$
\left(\frac{\lambda_{i}}{m_{i}} q_{i}-\frac{\lambda_{j}}{m_{j}} q_{j}\right) \wedge\left(q_{i}-q_{j}\right)=0
$$


Assim,

$$
-\frac{\lambda_{i}}{m_{i}} q_{i} \wedge q_{j}-\frac{\lambda_{j}}{m_{j}} q_{j} \wedge q_{i}=0
$$

o que implica em

$$
\left(\frac{\lambda_{i}}{m_{i}}-\frac{\lambda_{j}}{m_{j}}\right)\left(q_{j} \wedge q_{i}\right)=0 .
$$

Se $q_{j}$ é paralelo a $q_{i}$, a igualdade acima se verifica facilmente. Se $q_{i}$ e $q_{j}$ são não colineares, temos que

$$
\frac{\lambda_{i}}{m_{i}}=\frac{\lambda_{j}}{m_{j}}=\lambda
$$

para todo $i, j$. Portanto,

$$
\ddot{q}_{i}=\lambda q_{i}
$$

para todo $i=1,2, \ldots, n$, como queríamos provar.

Uma prova análoga pode ser feita para o seguinte teorema

Teorema 5.2.4 Considerando um sistema de $n$ massas não coplanares, então elas formam uma configuração central se e somente se respeitam a seguinte condição

$$
f_{i j h}=0, \quad \forall i, j, h(1 \leq i<j \leq n) e(h=1, \ldots, n) .
$$

Como uma primeira aplicação destas equações vamos seguir os trabalhos J. Bernat, J. Llibre e E. Pérez-Chavela em [45] no qual eles definem o que vem a ser configurações planares do tipo pipa no problema de quatro corpos. Os resultados aqui expostos podem ser encontrados em [46].

Estudaremos configurações centrais planares não colineares do problema de 4-corpos que têm a forma de pipa, ou simplesmente configurações centrais do tipo pipa, as quais podem ser definidas como àquelas que têm um eixo de simetria passando por duas das massas. A configuração do tipo pipa é chamada convexa se nenhum dos corpos está localizado no interior do fecho convexo dos outros três. Veja Figura 5.3. Caso contrário, dizemos que a configuração do tipo pipa é côncava. Veja Figura 5.4. 


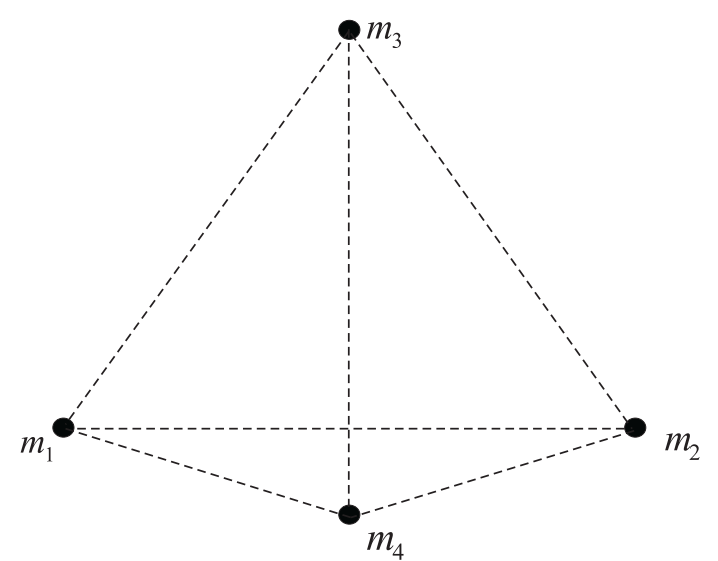

Figura 5.3: Pipa convexa.

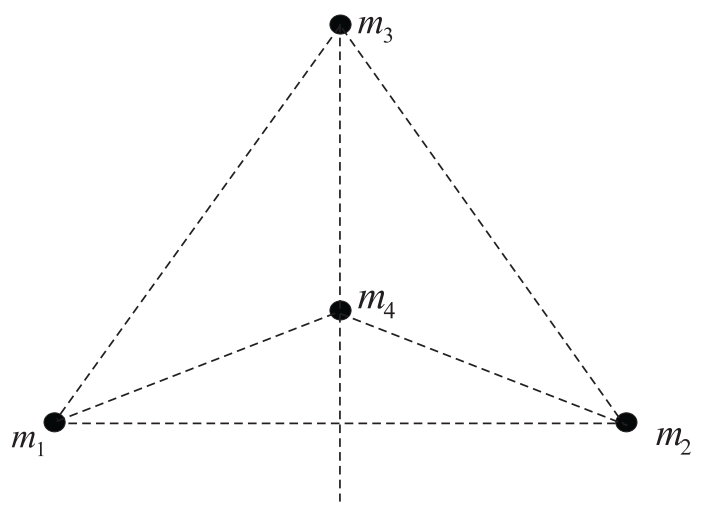

Figura 5.4: Pipa côncava.

Suporemos que as massas $m_{3}$ e $m_{4}$ estarão sobre a reta de simetria das configurações do tipo pipa, sendo que a posição de $m_{3}$ estará fixa, conforme a Figura 5.5. O resultado é o seguinte

Teorema 5.2.5 Considere quatro massas $m_{1}, m_{2}, m_{3}$ e $m_{4}$ localizadas em $(-x, 0),(x, 0),(0, \sqrt{3} / 2)$ $e(0, y)$, com $x>0$ e $y<\sqrt{3} / 2$, de acordo com a Figura 5.5. Valem as seguintes afirmações:

1. Para cada

$$
x_{0} \in\left(\frac{2 \sqrt{3}-3}{2}, \frac{1}{2}\right) \cup\left(\frac{1}{2}, \frac{2 \sqrt{3}+3}{2}\right),
$$

existe um intervalo aberto não vazio $I_{x_{0}}$ tal que, para cada $y_{0} \in I_{x_{0}}$, existem massas positivas $m_{1}=m_{2}, m_{3}$ e $m_{4}$ de modo que os quatro corpos, como na Figura 5.5, estão numa configuração central do tipo pipa;

2. Para $x_{0}=1 / 2$ e $y_{0}=\sqrt{3} / 6$, existem massas positivas $m_{1}=m_{2}=m_{3}$ e $m_{4}$ de modo que os quatro corpos, como na Figura 5.5, estão numa configuração central do tipo pipa côncava;

3. Para $x_{0}=\sqrt{3} / 2$ e $y_{0}=-\sqrt{3} / 2$, existem massas positivas $m_{1}=m_{2}=m_{3}=m_{4}$ de modo que os quatro corpos, como na Figura 5.5, estão numa configuração central do tipo pipa convexa.

\section{Prova:}

Nesta seção faremos a prova do Teorema 5.2.5 utilizando as equações de Dziobek 5.3. Para o problema planar de 4-corpos 5.3 é um conjunto de 6 equações. As configurações do tipo pipa, como 


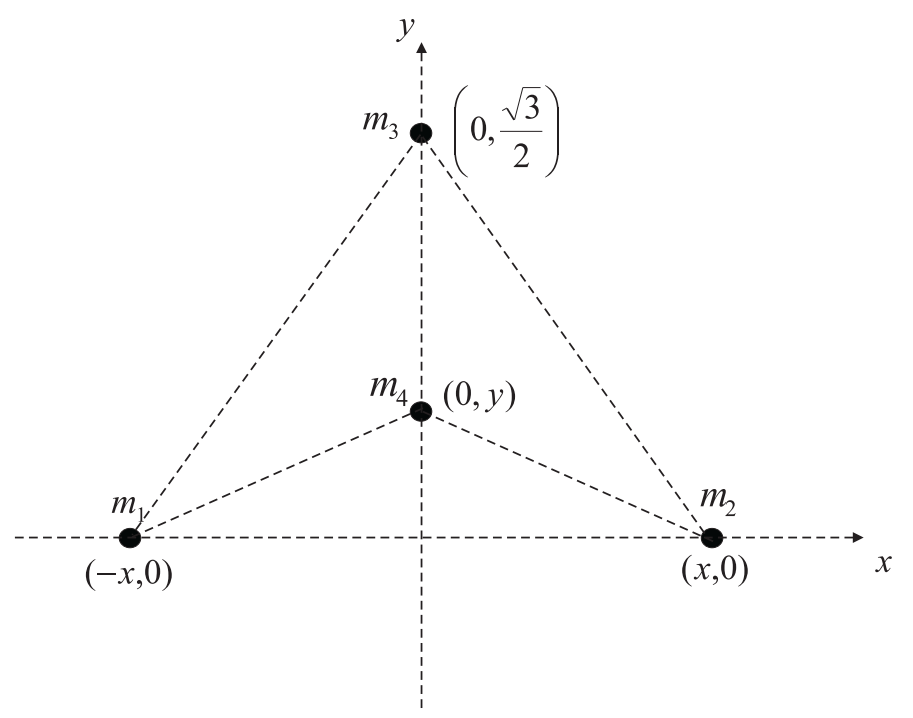

Figura 5.5: Configuração central do tipo Pipa.

na figura 5.5, sem colisões, devem satisfazer, entre outras, as seguintes relações

$$
r_{13}=r_{23}, \quad r_{14}=r_{24}, \quad \Delta_{143}=\Delta_{234}
$$

Consideremos as equações de Dziobek de nosso problema

$$
\begin{aligned}
& f_{12}=m_{3}\left(R_{13}-R_{23}\right) \Delta_{123}+m_{4}\left(R_{14}-R_{24}\right) \Delta_{124}=0, \\
& f_{13}=m_{2}\left(R_{12}-R_{32}\right) \Delta_{132}+m_{4}\left(R_{14}-R_{34}\right) \Delta_{134}=0, \\
& f_{14}=m_{2}\left(R_{12}-R_{42}\right) \Delta_{142}+m_{3}\left(R_{13}-R_{43}\right) \Delta_{143}=0, \\
& f_{23}=m_{1}\left(R_{21}-R_{31}\right) \Delta_{231}+m_{4}\left(R_{24}-R_{34}\right) \Delta_{234}=0, \\
& f_{24}=m_{1}\left(R_{21}-R_{41}\right) \Delta_{241}+m_{3}\left(R_{23}-R_{43}\right) \Delta_{243}=0, \\
& f_{34}=m_{1}\left(R_{31}-R_{41}\right) \Delta_{341}+m_{2}\left(R_{32}-R_{42}\right) \Delta_{342}=0 .
\end{aligned}
$$


Usando as relações acima, podemos reescrever a equação (5.20) da seguinte maneira

$$
\left(m_{1}-m_{2}\right)\left(R_{31}-R_{41}\right) \Delta_{341}=0
$$

Nas hipóteses do Teorema 5.2.5, o termo $\Delta_{134}$ nunca se anula, daí $m_{1}=m_{2}$ ou $R_{13}=R_{14}$. Se $R_{13}=R_{14}$, temos um quadrado, que é uma configuração central se, e somente se, $m_{1}=m_{2}=m_{3}=$ $m_{4}$ (veja item 3 do Teorema 5.2.5). Assim, podemos supor, sem perda de generalidade, que

$$
m_{1}=m_{2}=1
$$

A equação (5.15) é trivialmente satisfeita. Usando as relações de simetria acima, temos

$$
f_{13}=0 \Leftrightarrow f_{23}=0
$$

e

$$
f_{14}=0 \Leftrightarrow f_{24}=0 .
$$

Portanto, é suficiente encontrar soluções para (5.16) e (5.17), com valores positivos para as massas $m_{3}$ e $m_{4}$. Vamos reescrever estas equações de modo a obter $m_{3}$ e $m_{4}$ como funções das posições

$$
\begin{aligned}
& m_{3}=\frac{\left(R_{12}-R_{42}\right) \Delta_{142}}{\left(R_{43}-R_{13}\right) \Delta_{143}} \\
& m_{4}=\frac{\left(R_{12}-R_{32}\right) \Delta_{132}}{\left(R_{34}-R_{14}\right) \Delta_{134}} .
\end{aligned}
$$

Desejamos encontrar, se existirem, as regiões no semiplano $x>0$ para as quais teremos valores positivos para ambas as massas. Devemos, então, estudar o sinal dos termos envolvidos nas expressões de $m_{3}$ e $m_{4}$ em (5.21) e (5.22), respectivamente.

Substituindo as coordenadas, conforme indicações da Figura 5.6, temos

$$
\left(R_{12}-R_{42}\right)<0 \Leftrightarrow(x, y) \in\{x>0,-\sqrt{3} x<y<\sqrt{3} x\}
$$




$$
\begin{gathered}
\left(R_{34}-R_{14}\right)<0 \Leftrightarrow(x, y) \in\left\{x>0, y<-\frac{\sqrt{3}}{3} x^{2}+\frac{\sqrt{3}}{4}\right\}, \\
\left(R_{43}-R_{13}\right)<0 \Leftrightarrow(x, y) \in\left\{x>0, y<\frac{\sqrt{3}-\sqrt{3+4 x^{2}}}{2}\right\}, \\
\left(R_{12}-R_{32}\right)<0 \Leftrightarrow(x, y) \in\left\{x>\frac{1}{2}, y<\frac{\sqrt{3}}{2}\right\} .
\end{gathered}
$$

Para o sinal das áreas orientadas consideremos primeiramente $0<y<\sqrt{3} / 2$. Neste caso, nas expressões (5.21) e (5.22) vale

$$
\Delta_{142}<0, \quad \Delta_{143}>0, \quad \Delta_{132}<0, \quad \Delta_{134}<0
$$

Assim, comparando o sinal de cada um dos termos de (5.21) e (5.22), teremos que se $(x, y) \in A_{1} \cup A_{2}$, as massas $m_{3}$ e $m_{4}$ serão positivas, onde

$$
\begin{gathered}
A_{1}=\left\{(x, y) \in \mathbb{R}^{2}: \frac{2 \sqrt{3}-3}{2}<x<\frac{1}{2},-\frac{\sqrt{3}}{3} x^{2}+\frac{\sqrt{3}}{4}<y<\sqrt{3} x\right\}, \\
A_{2}=\left\{(x, y) \in \mathbb{R}^{2}: \frac{1}{2}<x<\frac{\sqrt{3}}{2}, 0<y<-\frac{\sqrt{3}}{3} x^{2}+\frac{\sqrt{3}}{4}\right\} .
\end{gathered}
$$

Consideremos agora $y<0$. Neste caso, nas expressões (5.21) e (5.22), temos

$$
\Delta_{142}>0, \quad \Delta_{143}>0, \quad \Delta_{132}<0, \quad \Delta_{134}<0 .
$$

Novamente, comparando o sinal de cada um dos termos de (5.21) e (5.22), teremos que se $(x, y) \in$ $A_{3}=B_{1} \cup B_{2}$, as massas $m_{3}$ e $m_{4}$ serão positivas, onde

$$
\begin{gathered}
B_{1}=\left\{(x, y) \in \mathbb{R}^{2}: \frac{1}{2}<x \leq \frac{3}{2},-\sqrt{3} x<y<\frac{\sqrt{3}-\sqrt{3+4 x^{2}}}{2}\right\}, \\
B_{2}=\left\{(x, y) \in \mathbb{R}^{2}: \frac{3}{2} \leq x \leq \frac{2 \sqrt{3}+3}{2},-\sqrt{3} x<y<-\frac{\sqrt{3}}{3} x^{2}+\frac{\sqrt{3}}{4}\right\} .
\end{gathered}
$$


O caso $y=0$ não precisa ser considerado, pois, não ocorre configuração central do tipo pipa, devido ao Teorema da Reta Perpendicular Bissetora, conforme [25].

Finalmente, concluímos que se $\left(x_{0}, y_{0}\right) \in A_{1} \cup A_{2} \cup A_{3}$, vide Figura 5.6, então as massas

$$
m_{1}=1, \quad m_{2}=1, \quad m_{3}=m_{3}\left(x_{0}, y_{0}\right), \quad m_{4}=m_{4}\left(x_{0}, y_{0}\right),
$$

com posições

$$
r_{1}=\left(-x_{0}, 0\right), \quad r_{2}=\left(x_{0}, 0\right), \quad r_{3}=\left(0, \frac{\sqrt{3}}{2}\right), \quad r_{4}=\left(0, y_{0}\right),
$$

formam uma configuração central do tipo pipa, como mostrada na Figura 5.6. O intervalo $I_{x_{0}}$ do enunciado do teorema é obtido tomando a interseção da reta $x=x_{0}$ com a região aberta $A_{i}$, $i=1,2,3$. Isso demonstra o item 1 do Teorema 5.2.5.

Para a prova do item 2, observemos que, neste caso, valem $r_{12}=r_{13}=r_{23}=1, r_{14}=r_{24}$ e $\Delta_{143}=\Delta_{234}$. Assim, $f_{12}=0$ é trivialmente satisfeita. De $f_{13}=0$ e de $f_{23}=0$ resultam, respectivamente, $R_{14}=R_{34}$ e $R_{24}=R_{34}$. Portanto, $R_{14}=R_{24}=R_{34}$. Assim, de $f_{24}=0$ e $f_{34}=0$, temos $m_{1}=m_{3}$ e $m_{1}=m_{2}$, respectivamente. Logo, $m_{1}=m_{2}=m_{3}$. De posse dessas conclusões sobre as massas e sobre as distâncias, segue que $f_{14}=0$ também é trivialmente satisfeita. Isso conclui a prova do item 2 do Teorema 5.2.5.

Passemos agora à prova do item 3 do Teorema 5.2.5. Neste caso, os 4 corpos estão sobre os vértices de um quadrado, de modo que as seguintes igualdades são obtidas: $r_{12}=r_{34}, r_{13}=r_{23}=$ $r_{14}=r_{24}$ e $\Delta_{123}=\Delta_{143}=\Delta_{234}=\Delta_{142}$. Substituindo essas informações nas equações de Dziobek resulta que $f_{12}=0$ e $f_{34}=0$ são trivialmente satisfeitas, enquanto que de $f_{13}=0, f_{14}=0$, $f_{23}=0$ e $f_{24}=0$ resultam $m_{2}=m_{4}, m_{2}=m_{3}, m_{1}=m_{4}$ e $m_{1}=m_{3}$, respectivamente. Portanto, $m_{1}=m_{2}=m_{3}=m_{4}$ e o item 3 do Teorema 5.2.5 está demonstrado.

Baseados no Teorema 5.2.5, seguem os seguintes corolários.

Corolário 5.2.6 Para cada $1 / 2<x_{0}<\sqrt{3} / 2$, existem dois intervalos abertos disjuntos $I_{x_{0}}^{+}$e $I_{x_{0}}^{-}$, tal que para cada $y_{0} \in I_{x_{0}}^{+} \cup I_{x_{0}}^{-}$, existem massas positivas $m_{1}=m_{2}, m_{3}$ e $m_{4}$, de modo que os quatro 


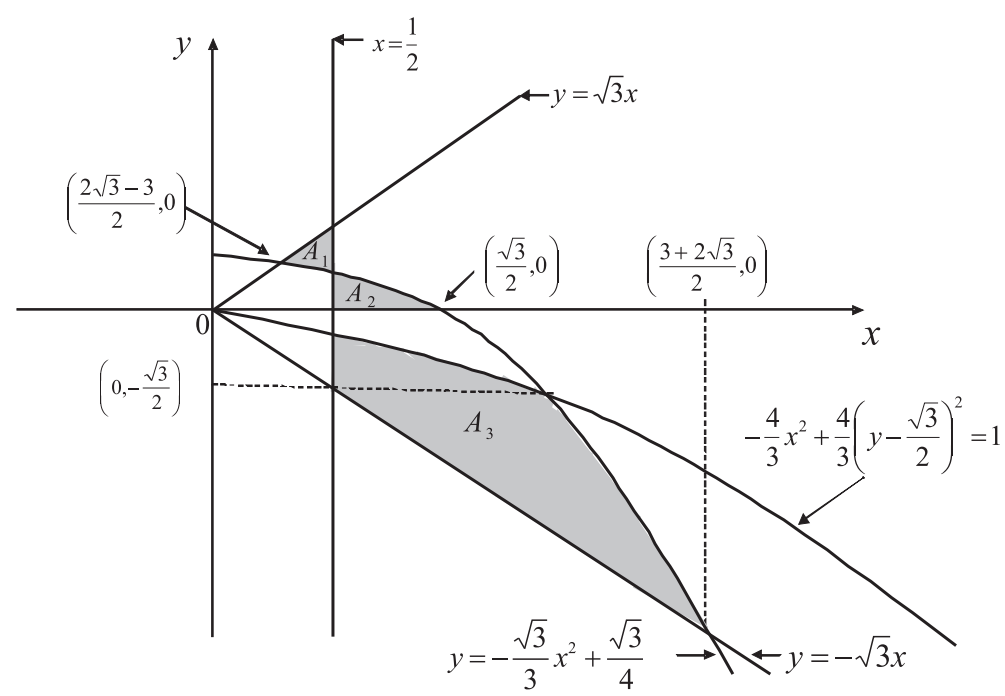

Figura 5.6: Regiões de existência de configurações centrais do tipo pipa.

corpos, como na Figura 5.5, estejam numa configuração central do tipo pipa. Mais especificamente, se $y_{0} \in I_{x_{0}}^{+}$a configuração central é do tipo pipa côncava e se $y_{0} \in I_{x_{0}}^{-}$a configuração central é do tipo pipa convexa.

Corolário 5.2.7 Considere $0<x<(2 \sqrt{3}-3) / 2$. Então não existem $y \in \mathbb{R}$ e massas positivas $m_{1}, m_{2}, m_{3}$ e $m_{4}$, de modo que os quatro corpos, como na Figura 5.5, estejam numa configuração central do tipo pipa.

Corolário 5.2.8 Considere $y<\sqrt{3} / 2$. Então, se $x>(2 \sqrt{3}+3) / 2$, não existem massas positivas $m_{1}, m_{2}, m_{3}$ e $m_{4}$, de modo que os quatro corpos, como na Figura 5.5, estejam numa configuração central do tipo pipa.

Um caso que pode interessar o leitor são as configurações centrais planares encaixantes, vide [47].

Vamos considerar agora alguns casos de configurações centrais espaciais, onde aplicaremos o segundo conjunto de equações de Dziobek 5.4. O próximo resultado pode ser encontrado em [48].

Primeiramente consideremos um problema de 5 corpos que de certa forma é uma generalização para o caso da pipa acima. Vamos supor as seguintes condições, vide 5.7(a) e 5.7(b):

1. O vetores posição $r_{1}, r_{2}$ e $r_{3}$ estão nos vértices de um triângulo equilátero $\mathcal{T}$; 


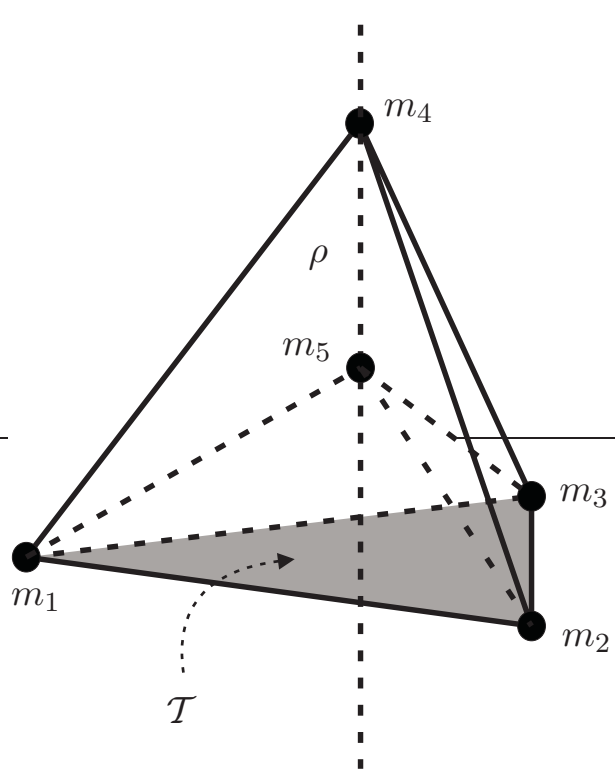

(a)

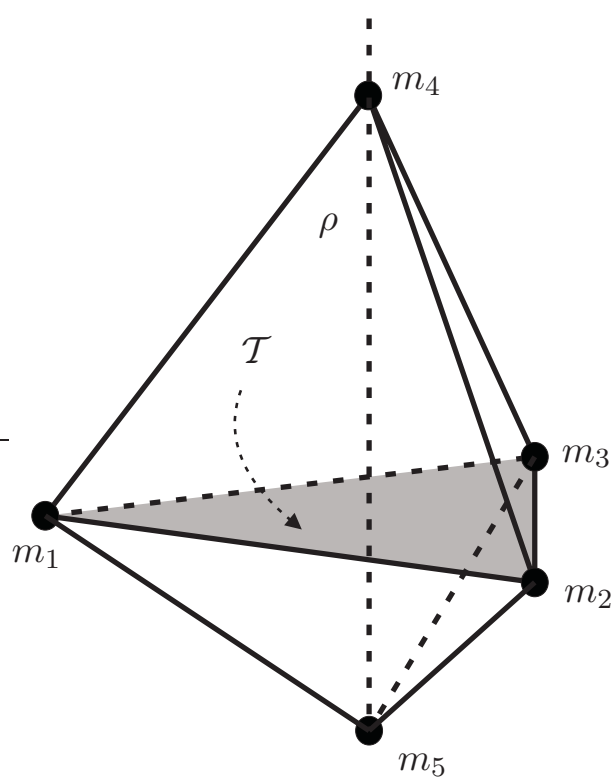

(b)

Figura 5.7: Configurações centrais espaciais para 5-corpos. Configuração Côncava (a). Configuração Convexa (b).

2. Defina a reta $\rho$ passando pelo baricentro de $\mathcal{T}$ perpendicular ao plano que contém $\mathcal{T}$. O vetor $r_{4} \in \rho$ está fixo e não pertence ao plano contendo $\mathcal{T}$;

3. $\mathrm{O}$ vetor $r_{5} \in \rho, r_{5} \neq r_{4}$.

Este tipo de configuração é chamada côncava se um dos corpos está no interior do fecho convexo dos outros quatro, vide figura 5.7(a). Caso contrário a configuração é chamada convexa, vide 5.7(b). Aqui nós diremos que uma configuração é côncava do tipo 1 se o corpo 5 está no interior do fecho convexo de 1, 2, 3 e 4, e diremos que a configuração é côncava do tipo 2 se o corpo 4 está no interior do fecho convexo de $1,2,3,5$.

Para simplificar a notação em nossos resultados, vamos considerar as seguintes coordenadas no nosso problema, $r_{1}=(x, 0,0), r_{2}=(-x / 2,-\sqrt{3} x / 2,0), r_{3}=(-x / 2, \sqrt{3} x / 2,0), r_{4}=(0,0, \sqrt{6} / 3) \mathrm{e}$ $r_{5}=(0,0, y) \operatorname{com} x>0$ e $y \neq \sqrt{6} / 3$, vide figura 5.8. Assim $x$ é o raio da circunferência circunscrita ao triângulo $\mathcal{T}$ e $y$ é a altura, com sinal, do quinto corpo com respeito ao plano que contém $\mathcal{T}$. Note que o quinto corpo estaria no baricentro de $\mathcal{T}$ se $y=0$. Por conseguinte, a configuração é convexa se e somente se $y \leq 0$. 


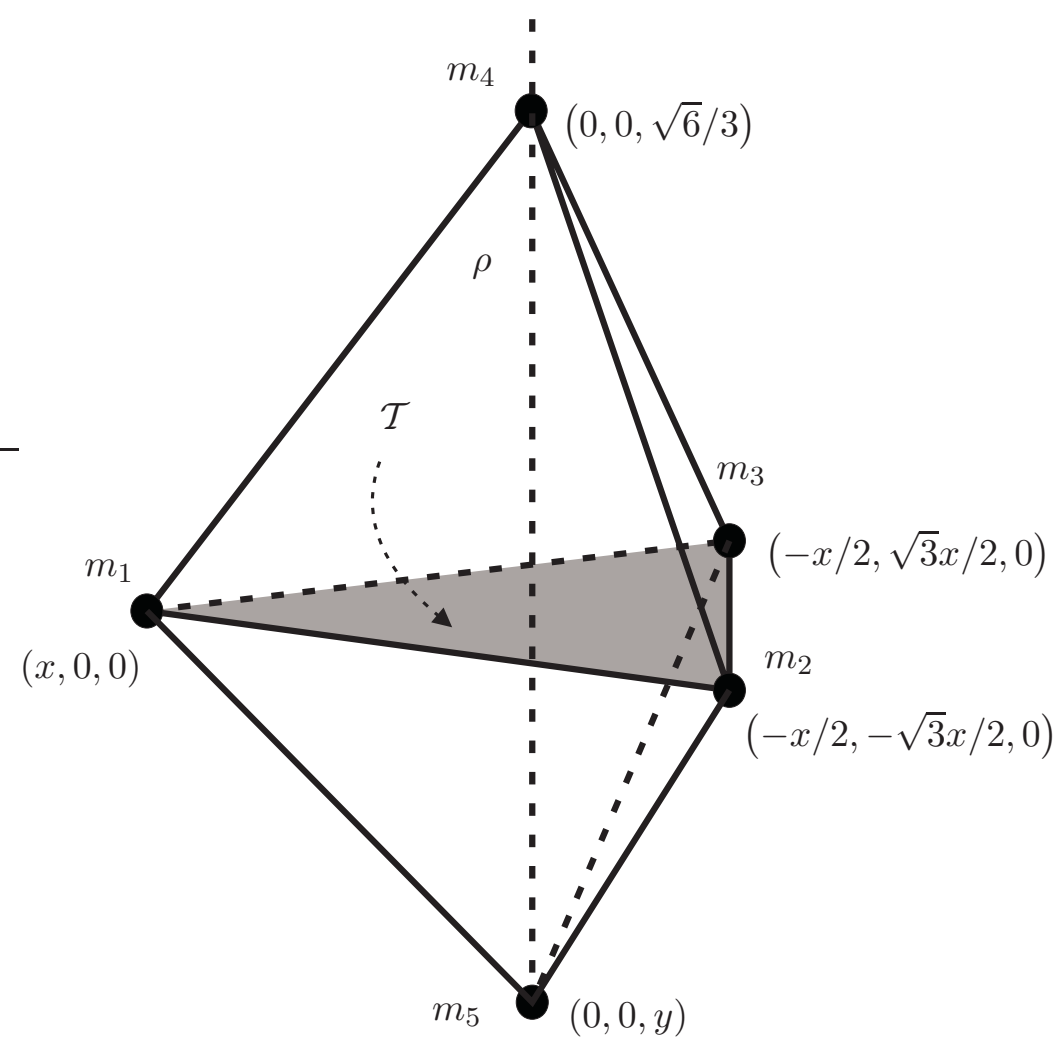

Figura 5.8: Coordenadas para o problema com 5 corpos.

Com estas considerações temos o seguinte resultado,

Teorema 5.2.9 Considere vetores posição nos vértices de um triângulo equilátero $\mathcal{T}, r_{1}=(x, 0,0)$, $r_{2}=(-x / 2,-\sqrt{3} x / 2,0), r_{3}=(-x / 2, \sqrt{3} x / 2,0)$ e vetores posição $r_{4}=(0,0, \sqrt{6} / 3), r_{5}=(0,0, y)$ sobre $\rho$, onde $\rho$ é a reta que passa pelo baricentro de $\mathcal{T}$ perpendicular ao plano que contém $\mathcal{T}$, de acordo com a figura 5.8. Assim,as seguintes afirmações são válidas:

1. Existe um valor mínimo $x=x_{\min }=(3 \sqrt{2}-2 \sqrt{3}) / 3$, tal que se $0<x \leq x_{\text {min }}$ não existem posições $r_{1}, \ldots, r_{5}$ nem massas positivas $m_{1}, \ldots, m_{5}$, tais que estes corpos formem uma configuração central;

2. Existem dois segmentos $I_{1}=((3 \sqrt{2}-2 \sqrt{3}) / 3, \sqrt{3} / 3)$ e $I_{2}=(\sqrt{3} / 3, \sqrt{6} / 3)$, tais que para cada $x \in I_{1} \cup I_{2}$ existe um segmento não vazio de possíveis valores para $y$, portanto possíveis posições para $r_{5}$ e massas positivas $m_{1}, \ldots, m_{5}$, tais que estes corpos formam uma família a um parâmetro de configurações centrais côncavas do tipo 1; 
3. Existe um valor distinguido e bem determinado $x=\bar{x}$ para o qual existe apenas uma posição $r_{5}$, tal que os corpos formam uma familia dois parâmetros de configurações centrais côncavas do tipo 1. Em verdade, esta configuração é exatamente aquela em que temos quatro massas iguais nos vértices de um tetraedro regular e o quinto corpo no baricentro deste tetraedro;

4. Existe um segmento $I_{3}=(\sqrt{3} / 3,(2 \sqrt{3}+3 \sqrt{2}) / 3)$, tal que para cada $x \in I_{3}$ existe um segmento não vazio de possiveis valores para $y$, portanto possiveis posições para $r_{5}$ e massas positivas $m_{1}, \ldots, m_{5}$, tais que estes corpos formam uma família a um parâmetro de configurações centrais convexas;

5. Existe um segmento ilimitado à direita $I_{4}=(4 \sqrt{3} / 3,+\infty)$, tal que para cada $x \in I_{4}$ existe um segmento não vazio de possíveis valores para $y$, portanto possíveis posições para $r_{5} e$ massas positivas $m_{1}, \ldots, m_{5}$, tais que estes corpos formam uma família a um parâmetro de configurações centrais côncavas do tipo 2;

6. Existe um valor distinguido e bem determinado $x=\bar{x}$ para o qual existe apenas uma posição $r_{5}$, tal que os corpos formam uma família dois parâmetros de configurações centrais côncavas do tipo 2. Em verdade, esta configuração é exatamente aquela em que temos quatro massas iguais nos vértices de um tetraedro regular e o quinto corpo no baricentro deste tetraedro.

Observação 5.2.10 Neste resultado os itens 2, 3 e 4 do teorema estão relacionados aos resultados obtidos por Leandro em [49], assim como os itens 3 e 6 podem ser encontrados em [34].

\section{Prova:}

Usaremos as equações de Dziobek com volumes, 5.6. Para cinco corpos teremos um sistema de 30 equações, mas existem algumas simetrias no nosso caso que simplificam os cálculos. Para as distâncias entre os corpos temos $R_{12}=R_{13}=R_{23}, R_{14}=R_{24}=R_{34}$ e $R_{15}=R_{25}=R_{35}$. Com nosso sistema de coordenadas temos $\Delta_{1234}>0$. Levando em conta as simetrias nos vem

$$
\begin{aligned}
& \Delta_{1425}=-\Delta_{1435}=-\Delta_{2415}=\Delta_{2435}=\Delta_{3415}=-\Delta_{3425}, \\
& \Delta_{1523}=-\Delta_{1532}=-\Delta_{2513}=\Delta_{2531}=\Delta_{3512}=-\Delta_{3521},
\end{aligned}
$$




$$
\Delta_{1524}=-\Delta_{1534}=-\Delta_{2514}=\Delta_{2534}=\Delta_{3514}=-\Delta_{3524} .
$$

Levando estas simetrias nas equações de Dziobek temos 9 equações de 5.4 trivialmente satisfeitas $f_{123}=0, f_{124}=0, f_{125}=0, f_{132}=0, f_{134}=0, f_{135}=0, f_{231}=0, f_{234}=0$ e $f_{235}=0$.

Ainda notando as simetrias, as seguintes equivalências entre equações são válidas,

$$
\begin{aligned}
& f_{145}=0 \Leftrightarrow f_{154}=0 \Leftrightarrow f_{451}=0 \Leftrightarrow\left(R_{12}-R_{24}\right) \Delta_{1452}\left(m_{2}-m_{3}\right)=0, \\
& f_{245}=0 \Leftrightarrow f_{254}=0 \Leftrightarrow f_{452}=0 \Leftrightarrow\left(R_{12}-R_{14}\right) \Delta_{2451}\left(m_{1}-m_{3}\right)=0, \\
& f_{345}=0 \Leftrightarrow f_{354}=0 \Leftrightarrow f_{453}=0 \Leftrightarrow\left(R_{13}-R_{14}\right) \Delta_{3451}\left(m_{1}-m_{2}\right)=0 .
\end{aligned}
$$

Daí as 21 equações restantes de 5.4 são reduzidas a 15 equações. Como nossas classes de configurações centrais satisfazem $r_{4} \neq r_{5}$ temos $\Delta_{1452} \neq 0, \Delta_{2451} \neq 0$ e $\Delta_{3451} \neq 0$. Das equações 5.23, 5.24 e 5.25 temos que analisar dois casos: quando as massas $m_{1}=m_{2}=m_{3}$ e quando $r_{1}, r_{2}, r_{3}$ e $r_{4}$ estão nos vértices de um tetraedro regular.

Caso 1. Considere $r_{1}, r_{2}, r_{3}$ e $r_{4}$ nos vértices de um tetraedro regular. Temos então o seguinte lema,

Lema 5.2.11 Considere $r_{1}, r_{2}, r_{3}$ e $r_{4}$ nos vértices de um tetraedro regular. Então, existe apenas uma posição $r_{5}$ no centro de massa do tetraedro e massas positivas $m_{1}=m_{2}=m_{3}=m_{4}=m e$ $m_{5}$, tais que os corpos formam uma família a dois parâmetros de configurações centrais côncavas do tipo 1.

Prova. Devido às simetrias as seguintes equações são equivalentes em 5.4

$$
\begin{aligned}
& f_{142}=0 \Leftrightarrow f_{241}=0, \quad f_{143}=0 \Leftrightarrow f_{341}=0, \quad f_{243}=0 \Leftrightarrow f_{342}=0, \\
& f_{152}=0 \Leftrightarrow f_{251}=0, \quad f_{153}=0 \Leftrightarrow f_{351}=0, \quad f_{253}=0 \Leftrightarrow f_{352}=0 .
\end{aligned}
$$

Daí as 15 equações restantes são reduzidas a 9 equações. De $f_{142}=0, f_{143}=0,2 f_{243}=0$ temos $m_{5}\left(R_{15}-R_{45}\right) \Delta_{1425}=0, m_{5}\left(R_{15}-R_{45}\right) \Delta_{1435}=0$ e $m_{5}\left(R_{25}-R_{45}\right) \Delta_{2435}=0$, respectivamente. 
As $\Delta_{1425} \neq 0, \Delta_{1435} \neq 0$ e $\Delta_{2435} \neq 0$ segue que $R_{15}=R_{45}=R_{25}$. Isto implica que $r_{5}$ deve estar no centro de massa do tetraedro. Levando esta informação em $f_{152}=0, f_{153}=0$ e $f_{253}=0$ temos $\left(m_{3}-m_{4}\right)\left(R_{13}-R_{35}\right) \Delta_{1523}=0,\left(m_{2}-m_{4}\right)\left(R_{12}-R_{25}\right) \Delta_{1532}=0$ e $\left(m_{1}-m_{4}\right)\left(R_{12}-R_{15}\right) \Delta_{2531}=$ 0, respectivamente. Estas 3 últimas equações são satisfeitas somente quando $m_{1}=m_{2}=m_{3}=m_{4}$. Por sua vez $m_{5}$ pode assumir qualquer valor positivo. As outras 3 equações restantes $f_{145}=0$, $f_{245}=0$ e $f_{345}=0$ são trivialmente verificadas. Assim o lema está provado.

Defina $\bar{x}=\sqrt{3} / 3 . \quad$ Assim $r_{1}=(\sqrt{3} / 3,0,0), r_{2}=(-\sqrt{3} / 6,-1 / 2,0), r_{3}=(-\sqrt{3} / 6,1 / 2,0)$, $r_{4}=(0,0, \sqrt{6} / 3)$ e $r_{5}=(0,0, \sqrt{6} / 12)$. O lema 5.2 .11 prova item 3 do teorema 5.2.9. No restante desta prova omitiremos esta situação de configuração central côncava.

Caso 2. Considere $m_{1}=m_{2}=m_{3}=m$. Então as equações $5.23,5.24$ e 5.25são satisfeitas e

$$
\begin{aligned}
& f_{142}=0 \Leftrightarrow f_{143}=0 \Leftrightarrow f_{241}=0 \Leftrightarrow f_{243}=0 \Leftrightarrow f_{341}=0 \Leftrightarrow f_{342}=0, \\
& f_{152}=0 \Leftrightarrow f_{153}=0 \Leftrightarrow f_{251}=0 \Leftrightarrow f_{253}=0 \Leftrightarrow f_{351}=0 \Leftrightarrow f_{352}=0 .
\end{aligned}
$$

Noutros termos, as 30 equações foram reduzidas a apenas 2, as quais seguem

$$
\begin{aligned}
& f_{142}=m\left(R_{13}-R_{34}\right) \Delta_{1423}+m_{5}\left(R_{15}-R_{45}\right) \Delta_{1425}=0, \\
& f_{152}=m\left(R_{13}-R_{35}\right) \Delta_{1523}+m_{4}\left(R_{14}-R_{45}\right) \Delta_{1524}=0 .
\end{aligned}
$$

As equações 5.26 e 5.27 podem ser resolvidas diretamente de forma a termos $m_{4}=m_{4}(x, y, m)$ e $m_{5}=m_{5}(x, y, m)$, respectivamente. Nestas equações $m$ pode ser entendido como um parâmetro para as configurações centrais.

Da equação 5.26 e 5.27 temos

$$
\frac{m_{4}}{m}=\frac{\left(R_{35}-R_{13}\right) \Delta_{1523}}{\left(R_{14}-R_{45}\right) \Delta_{1524}}
$$




$$
\frac{m_{5}}{m}=\frac{\left(R_{34}-R_{13}\right) \Delta_{1423}}{\left(R_{15}-R_{45}\right) \Delta_{1425}}
$$

O que precisamos determinar são os subconjuntos de $\mathcal{D}=\{x>0, y \in \mathbb{R}, y \neq \sqrt{6} / 3\}$ onde ambas as razões de massas $m_{4} / m$ e $m_{5} / m$ são positivas. Para o estudo dos sinais os termos que aparecem nas expressões 5.28 e 5.29 temos $R_{35}-R_{13}=0$ se e só se $(x, y) \in\{x>0, y=-\sqrt{2} x\} \cup\{x>$ $0, y=\sqrt{2} x\}$ (Retas), $R_{14}-R_{45}=0$ se e só se $(x, y) \in\left\{x>0, y=\sqrt{6} / 3-\sqrt{9 x^{2}+6} / 3\right\} \cup\{x>$ $\left.0, y=\sqrt{6} / 3+\sqrt{9 x^{2}+6} / 3\right\}$ (Ramo de Hipérbole), $R_{34}-R_{13}=0$ se e só $\operatorname{se}(x, y) \in\{x=\sqrt{3} / 3\}$, $R_{15}-R_{45}=0$ se e só se $(x, y) \in\left\{x>0, y=\sqrt{6}\left(2-3 x^{2}\right) / 12\right\}$ (Parábola), $\Delta_{1523}=0$ se e só se $(x, y) \in\{x>0, y=0\}, \Delta_{1524}=0$ se e só se $(x, y) \in\{x>0, y=\sqrt{6} / 3\}, \Delta_{1425}=0$ se e só se $(x, y) \in\{x>0, y=\sqrt{6} / 3\}$. Veja figuras 5.9 e 5.10 .

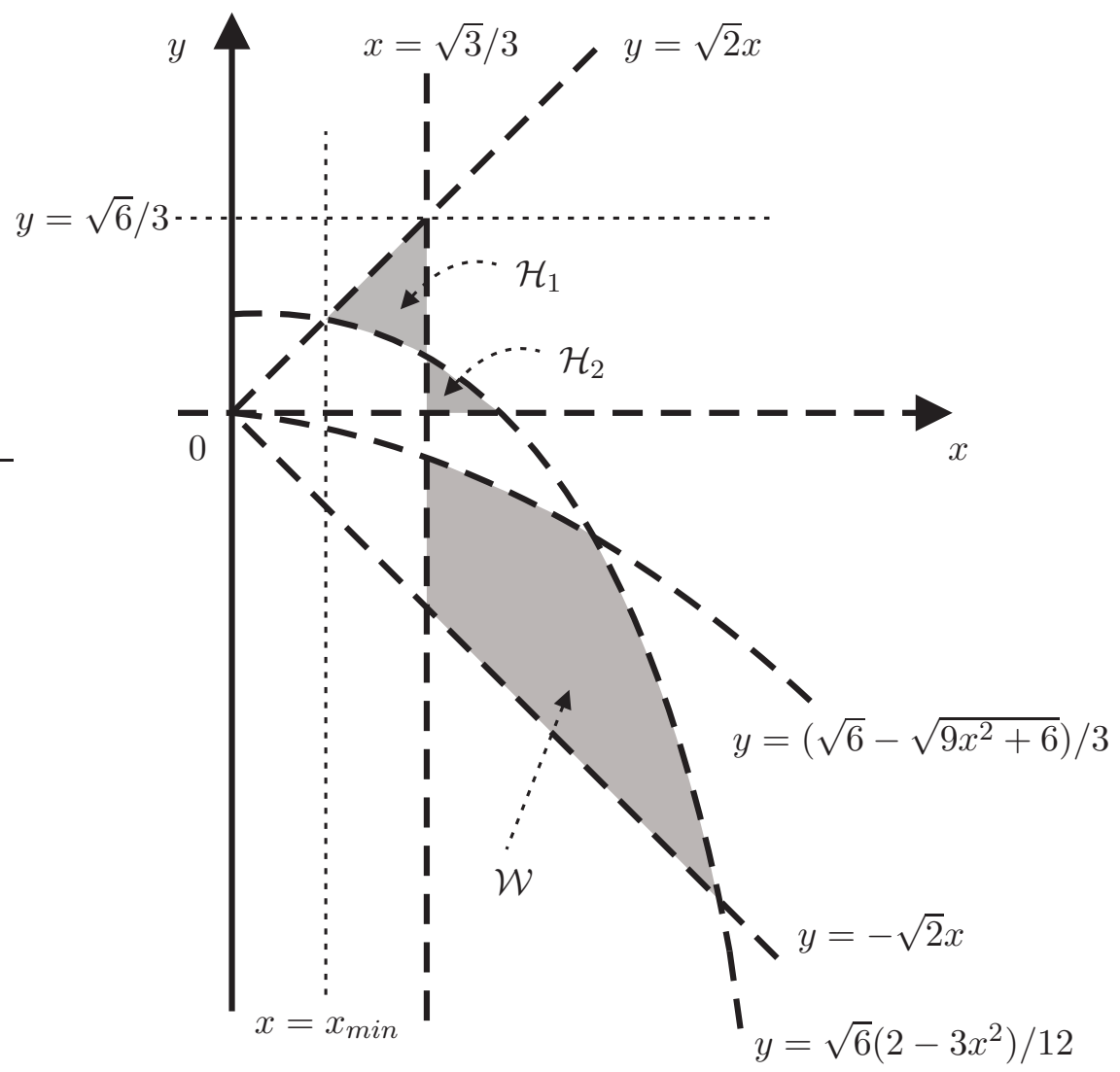

Figura 5.9: Regiões abertas $\mathcal{H}_{1}, \mathcal{H}_{2}$ e $\mathcal{W}$.

Caso 2.1. Considere $x>0$ e $y=0$. 
Lema 5.2.12 Considere $r_{5}=(0,0,0)$, que é $y=0$. Então, não existe $x>0$ tal que os 5 corpos com massas positivas formem uma configuração central de acordo com a figura 5.8.

Prova. Das equações 5.27 temos

$$
m\left(R_{13}-R_{35}\right) \Delta_{1523}+m_{4}\left(R_{14}-R_{45}\right) \Delta_{1524}=0 .
$$

Por hipótese $\Delta_{1523}=0$. Como $\left(R_{14}-R_{45}\right) \neq 0$ e $\Delta_{1524} \neq 0$ então $m_{4}=0$. Isto é uma contradição.

Caso 2.2. Considere $0<x \leq(3 \sqrt{2}-2 \sqrt{3}) / 3$ e $y \neq \sqrt{6} / 3$. Temos o seguinte lema.

Lema 5.2.13 Considere $0<x \leq(3 \sqrt{2}-2 \sqrt{3}) / 3$. Então não existe posição para o corpo 5 sobre $\rho$ e massas positivas $m_{i}, i=1, \ldots, 5$, tais que estes corpos formem uma configuração central de acordo com a figura 5.8 .

Prova. Devemos analisar três casos: $y<0,0<y<\sqrt{6} / 3$ e $y>\sqrt{6} / 3$.

Considere $y<0$. Da equação 5.26 temos

$$
m\left(R_{13}-R_{34}\right) \Delta_{1423}+m_{5}\left(R_{15}-R_{45}\right) \Delta_{1425}=0 .
$$

Por hipótese $R_{13}-R_{34}<0, R_{15}-R_{45}<0, \Delta_{1423}>0$ e $\Delta_{1425}>0$. Por conseguinte, os coeficientes da expressão acima tem o mesmo sinal. Para que se anule precisamos que $m_{5}$ e $m$ tenham sinais opostos. Isto é uma contradição.

Considere $0<y<\sqrt{6} / 3$. Por hipótese $R_{13}-R_{34}<0, R_{13}-R_{35}>0, R_{14}-R_{45}<0, \Delta_{1423}>0$, $\Delta_{1425}>0, \Delta_{1523}>0, \Delta_{1524}<0, R_{15}-R_{45}<0$, se $0<y<\sqrt{6}\left(2-3 x^{2}\right) / 12$ e $R_{15}-R_{45}>0$, se $\sqrt{6}\left(2-3 x^{2}\right) / 12<y<\sqrt{6} / 3$.

Da equação 5.26 temos

$$
m\left(R_{13}-R_{34}\right) \Delta_{1423}+m_{5}\left(R_{15}-R_{45}\right) \Delta_{1425}=0 .
$$


Se $0<y<\sqrt{6}\left(2-3 x^{2}\right) / 12$ então os coeficientes da expressão acima acima têm o mesmo sinal, portanto para que se anule é preciso que $m_{5}$ e $m$ tenham sinais opostos. Isto é uma contradição.

Considere agora a equação 5.27 temos

$$
m\left(R_{13}-R_{35}\right) \Delta_{1523}+m_{4}\left(R_{14}-R_{45}\right) \Delta_{1524}=0 .
$$

Se $\sqrt{6}\left(2-3 x^{2}\right) / 12<y<\sqrt{6} / 3$ então os coeficientes da expressão acima acima têm o mesmo sinal, portanto para que se anule é preciso que $m_{4}$ e $m$ tenham sinais opostos. Isto é uma contradição. Se $y=\sqrt{6}\left(2-3 x^{2}\right) / 12$ então $R_{15}-R_{45}=0$ mas isto implica que $m=0$ na equação 5.26 . Novamente temos contradição.

Considere $y>\sqrt{6} / 3$. Da equação 5.26 temos

$$
m\left(R_{13}-R_{34}\right) \Delta_{1423}+m_{5}\left(R_{15}-R_{45}\right) \Delta_{1425}=0 .
$$

Por hipótese $R_{13}-R_{34}<0, R_{15}-R_{45}>0, \Delta_{1423}>0$ e $\Delta_{1425}<0$. Por conseguinte os coeficientes da expressão acima acima têm o mesmo sinal, portanto para que se anule é preciso que $m_{5}$ e $m$ tenham sinais opostos. Isto é uma contradição.

Do lema 5.2.13 provamos o item 1 do teorema 5.2.9. O valor $x_{\min }=(3 \sqrt{2}-2 \sqrt{3}) / 3$ é definido como a coordenada $x$ da interseção das curvas $R_{35}-R_{13}=0$ e $R_{15}-R_{45}=0$, vide figura 5.9.

Caso 2.3. Considere $x>0$ e $0<y<\sqrt{6} / 3$.

Defina $\mathcal{H}=\mathcal{H}_{1} \cup \mathcal{H}_{2}$, vide figura 5.9, onde

$$
\begin{gathered}
\mathcal{H}_{1}=\left\{\frac{3 \sqrt{2}-2 \sqrt{3}}{3}<x<\frac{\sqrt{3}}{3}, \frac{\sqrt{6}\left(2-3 x^{2}\right)}{12}<y<\sqrt{2} x\right\}, \\
\mathcal{H}_{2}=\left\{\frac{\sqrt{3}}{3}<x<\frac{\sqrt{6}}{3}, 0<y<\frac{\sqrt{6}\left(2-3 x^{2}\right)}{12}\right\} .
\end{gathered}
$$


Para $(x, y) \in \mathcal{H}_{1}$ temos $x>0,0<y<\sqrt{6} / 3, R_{35}-R_{13}>0, R_{34}-R_{13}<0, R_{15}-R_{45}<0$, $R_{14}-R_{45}<0, \Delta_{1523}>0, \Delta_{1524}<0, \Delta_{1423}>0$ e $\Delta_{1425}>0$. Para $(x, y) \in \mathcal{H}_{2}$ temos $x>0$, $0<y<\sqrt{6} / 3, R_{35}-R_{13}>0, R_{34}-R_{13}>0, R_{15}-R_{45}>0, R_{14}-R_{45}<0, \Delta_{1523}>0, \Delta_{1524}<0$, $\Delta_{1423}>0$ e $\Delta_{1425}>0$.

É fácil ver que para $(x, y) \in \mathcal{H}$ o lado direito das expressões 5.28 e 5.29 é positivo, portanto temos configurações centrais côncavas do tipo 1 .

As projeções ortogonais dos conjuntos abertos $\mathcal{H}_{1}$ e $\mathcal{H}_{2}$ sobre o eixo $x$ nos dá dois segmentos $I_{1}=((3 \sqrt{2}-2 \sqrt{3}) / 3, \sqrt{3} / 3)$ e $I_{2}=(\sqrt{3} / 3, \sqrt{6} / 3)$, respectivamente. Para cada $x_{*} \in I_{1} \cup I_{2}$ a reta $x=x_{*}$ intersecta $\mathcal{H}_{1} \cup \mathcal{H}_{2}$ em um segmento não vazio. Isto prova o item 2 do teorema 5.2.9.

Caso 2.4. Considere $x>0$ e $y<0$.

Para termos configurações centrais convexas é necessário que $y<0$. Defina o conjunto aberto $\mathcal{W}=\mathcal{W}_{1} \cup \mathcal{W}_{2}$, vide figura 5.9 , onde

$$
\begin{gathered}
\mathcal{W}_{1}=\left\{\frac{\sqrt{3}}{3}<x \leq \sqrt{2},-\sqrt{2} x<y<\frac{\sqrt{6}}{3}-\frac{\sqrt{9 x^{2}+6}}{3}\right\}, \\
\mathcal{W}_{2}=\left\{\sqrt{2} \leq x<\frac{2 \sqrt{3}+3 \sqrt{2}}{3},-\sqrt{2} x<y<\frac{\sqrt{6}\left(2-3 x^{2}\right)}{12}\right\} .
\end{gathered}
$$

Para $(x, y) \in \mathcal{W}$ temos $x>0, y<0, R_{35}-R_{13}>0, R_{34}-R_{13}>0, R_{15}-R_{45}>0, R_{14}-R_{45}>0$, $\Delta_{1523}<0, \Delta_{1524}<0, \Delta_{1423}>0$ e $\Delta_{1425}>0$.

É fácil ver que para $(x, y) \in \mathcal{W}$ o lado direito das expressões 5.28 e 5.29 é positivo e por conseguinte temos configurações centrais convexas.

A projeção ortogonal de $\mathcal{W}$ sobre o eixo $x$ nos dá um segmento $I_{3}=(\sqrt{3} / 3,(2 \sqrt{3}+3 \sqrt{2}) / 3)$. Para cada $x_{*} \in I_{3}$ a reta $x=x_{*}$ intersecta $\mathcal{W}$ em um segmento não vazio. Isto prova o item 4 do teorema 5.2.9.

Caso 2.5. Considere $x>0$ e $y>\sqrt{6} / 3$.

Defina seguinte região ilimitada aberta, vide figura 5.10,

$$
\mathcal{U}=\left\{\frac{4 \sqrt{3}}{3}<x, \frac{\sqrt{6}+\sqrt{9 x^{2}+6}}{3}<y<\sqrt{2} x\right\}
$$




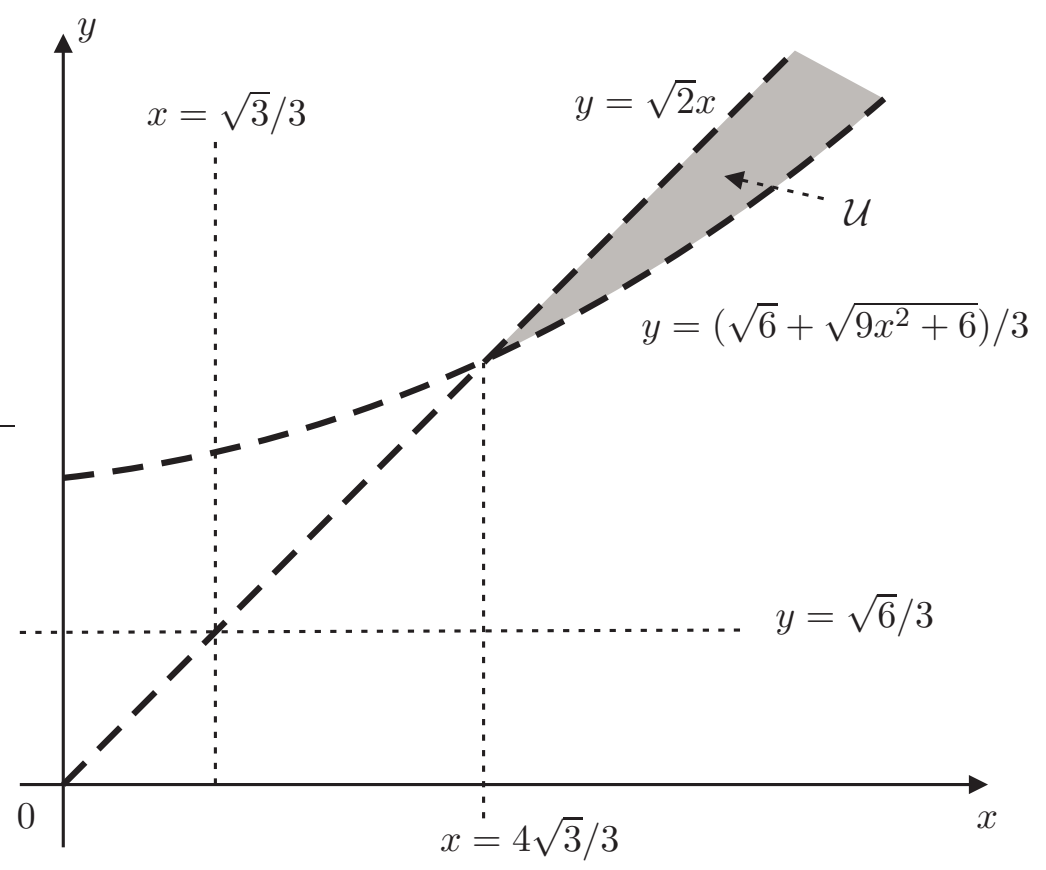

Figura 5.10: Região aberta $\mathcal{U}$.

Para $(x, y) \in \mathcal{U}$ temos $x>0, y>\sqrt{6} / 3, R_{35}-R_{13}>0, R_{34}-R_{13}>0, R_{15}-R_{45}<0, R_{14}-R_{45}>0$, $\Delta_{1523}>0, \Delta_{1524}>0, \Delta_{1423}>0$ e $\Delta_{1425}<0$.

É fácil ver que para $(x, y) \in \mathcal{U}$ o lado direito das expressões 5.28 e 5.29 é positivo e por conseguinte temos configurações centrais côncavas do tipo 2 .

A projeção ortogonal de $\mathcal{U}$ sobre o eixo $x$ nos dá o segmento ilimitado $I_{4}=(4 \sqrt{3} / 3,+\infty)$. Para cada $x_{*} \in I_{4}$ a reta $x=x_{*}$ intersecta $\mathcal{U}$ em um segmento não vazio. Isto prova o item 5 do teorema 5.2 .9 .

Caso 2.6. Considere $r_{1}, r_{2}, r_{3}$ e $r_{5}$ nos vértices de um tetraedro regular. Temos o seguinte lema,

Lema 5.2.14 Se $m_{1}=m_{2}=m_{3}=m_{5}=m$ e $m_{4}$ estão em $r_{1}=(4 \sqrt{3} / 3,0,0), r_{2}=(-2 \sqrt{3} / 3,-2,0)$, $r_{3}=(-2 \sqrt{3} / 3,2,0), r_{4}=(0,0, \sqrt{6} / 3)$ e $r_{5}=(0,0,4 \sqrt{6} / 3)$ então estes corpos formam uma familia a dois parâmetros de configurações centrais côncavas do tipo 2.

Prova. Por hipótese $x=4 \sqrt{3} / 3$ e $y=4 \sqrt{6} / 3$. Para estes valores temos $R_{13}-R_{35}=0$ e $R_{14}-R_{45}=0$. Por conseguinte, a expressão 5.27 é satisfeita para todo $m_{4}>0$. Como $m_{5}=m$ a 
equação 5.26 pode ser escrita como

$$
m\left[\left(R_{13}-R_{34}\right) \Delta_{1423}+\left(R_{15}-R_{45}\right) \Delta_{1425}\right]=0 .
$$

Com estes valores para $x$ e $y$ temos $r_{13}=r_{15}=4, r_{34}=r_{45}=\sqrt{6}, \Delta_{1423}=-\Delta_{1425}=8 \sqrt{2}$. Por um cálculo direto vemos que $\left(R_{13}-R_{34}\right) \Delta_{1423}+\left(R_{15}-R_{45}\right) \Delta_{1425}=0$. É fácil ver que $r_{1}, r_{2}, r_{3} \mathrm{e}$ $r_{5}$ estão nos vértices de um tetraedro regular e que $r_{4}$ está no baricentro do tetraedro.

A prova do item 6 do teorema 5.2.9 segue do lema 5.2.14 onde $\overline{\bar{x}}=4 \sqrt{3} / 3$. Isto finda a prova do teorema.

Um caso de configuração central espacial parecido e com prova similar este é o próximo teorema para um problema de seis corpos o qual respeita a seguinte construção

1. Os vetores posição $r_{1}, r_{2}$ e $r_{3}$ estão nos vértices de um triângulo equilátero $\mathcal{T}$ cujos lados tem comprimento $a$;

2. Seja $\mathcal{L}$ a linha passando pelo baricentro de $\mathcal{T}$ e perpendicular ao plano que contém $\mathcal{T}$. Os vetores posição $r_{4}, r_{5}, r_{6} \in \mathcal{L}$. Mais especificamente, o vetor $r_{6}$ está fixo no baricentro de $\mathcal{T}$ e os vetores $r_{4}$ e $r_{5}$ estão localizados simetricamente com respeito ao plano que contém $\mathcal{T}$.

Com estas considerações temos o seguinte resultado.

Teorema 5.2.15 Assuma três corpos com massas $m_{1}, m_{2}$ e $m_{3}$ nos vértices de um triângulo equilátero $\mathcal{T}$ cujos lados tem comprimento fixo a, dois corpos com massas $m_{4}$ e $m_{5}$ estão localizados simetricamente sobre a linha $\mathcal{L}$ passando pelo baricentro de $\mathcal{T}$ e perpendicular ao plano que contém $\mathcal{T}$ e um corpo com massa $m_{6}$ no baricentro de $\mathcal{T}$ de acordo com a figura 5.11. Para que este seis corpos formem uma configuração central as seguintes afirmações devem ser satisfeitas:

(a) As três massas $m_{1}, m_{2}$ e $m_{3}$ são iguais; 


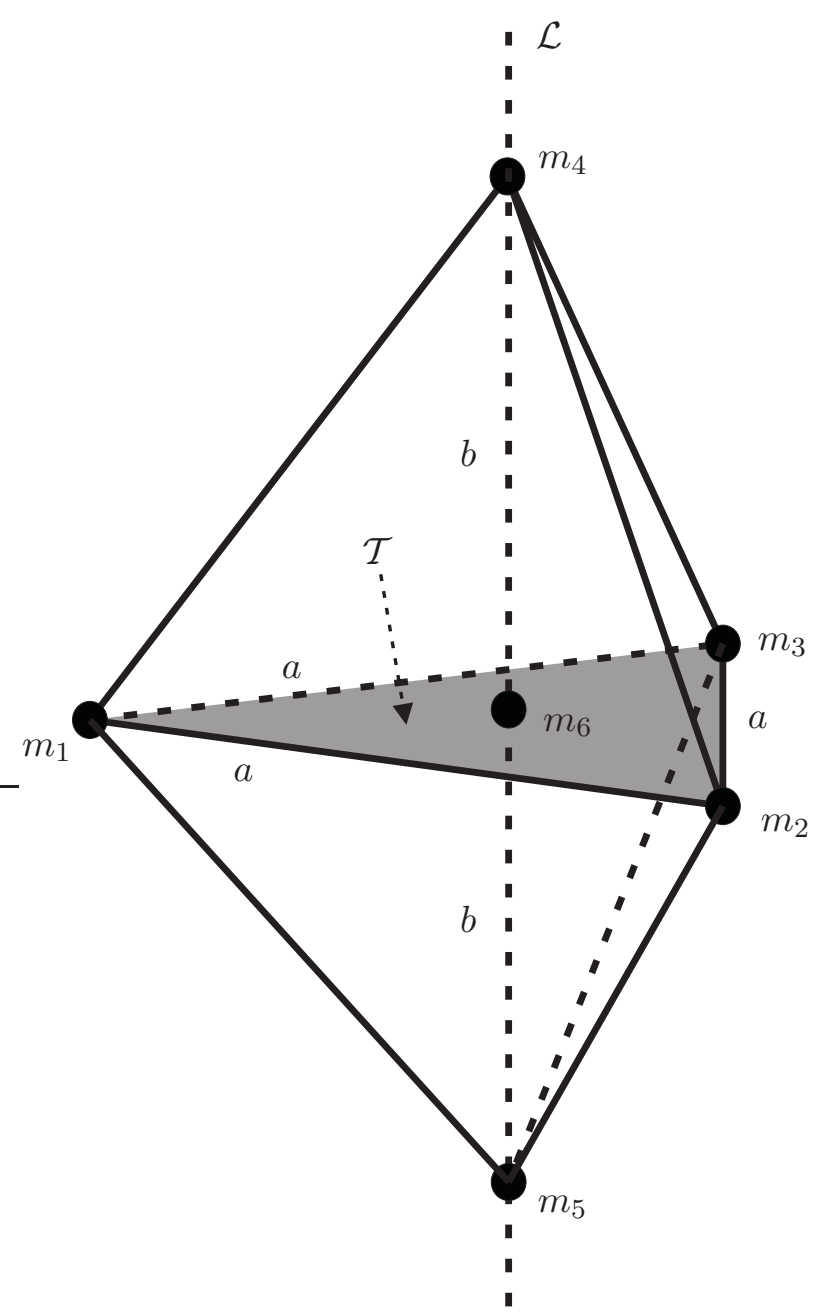

Figura 5.11: Os corpos com massas $m_{1}, m_{2}$ e $m_{3}$ estão nos vértices de um triângulo equilátero $\mathcal{T}$, o corpo com massa $m_{6}$ está no baricentro de $\mathcal{T}$ e os corpos com massas $m_{4}, m_{5}$ estão localizados simetricamente sobre $\mathcal{L}$.

(b) As duas massas $m_{4}$ e $m_{5}$ são iguais.

Seja $b$ a distância entre os corpos com massas $m_{4}$ e $m_{6}$ (veja a figura 5.11).

1. Existe um intervalo não vazio $I_{1} \subset \mathbb{R}$ tal que, para cada $b \in I_{1}$ existem vetores posição $r_{i}$, $i=1, \ldots, 6$ e massas positivas $m_{1}=m_{2}=m_{3}, m_{4}=m_{5}$ e $m_{6}$, tais que os seis corpos estão em uma configuração central;

2. Suponha que $m_{1}=m_{2}=m_{3}=m_{4}=m_{5}=m$. Então existe um intervalo não vazio $I_{2} \subset \mathbb{R}$ tal que, para cada $b \in I_{2}$ existem vetores posição $r_{i}, i=1, \ldots, 6$ e massas positivas $m$ e $m_{6}$ 
tais que os seis corpos estão em uma configuração central;

3. Suponha que $m_{1}=m_{2}=m_{3}=m_{4}=m_{5}=m_{6}=M$. Então existe um único valor para $b_{*} \in I_{2}$ tal que, os seis corpos estão em uma configuração central.

A prova deste teorema pode ser encontrada em [50].

Existem muitos problemas em aberto na linha das configurações centrais do problema, principalmente no caso espacial.

Observando as provas dos teoremas 5.2.5 e 5.2.9 é notável a semelhança entre as figuras 5.6 e 5.9. De fato, no problema planar também temos uma região ilimitada semelhante àquela mostrada na figura 5.10. Uma pergunta natural é: Substituindo o triângulo equilátero da base por um outro polígono regular o resultado é, ainda, similar? Se a resposta for afirmativa temos de encontrar uma prova simples para a mesma.

Conhecidas as configurações podemos construir soluções homográficas tomando a velocidade de cada massa perpendicular ao vetor posição, no centro de massa, e de tal forma que essas velocidades tenham módulos proporcionais, com mesma constante de proporcionalidade, ao módulo do vetor posição [51]. Desta forma todas as massas farão órbitas cônicas em torno do centro de massa, vide figura 5.12 . 


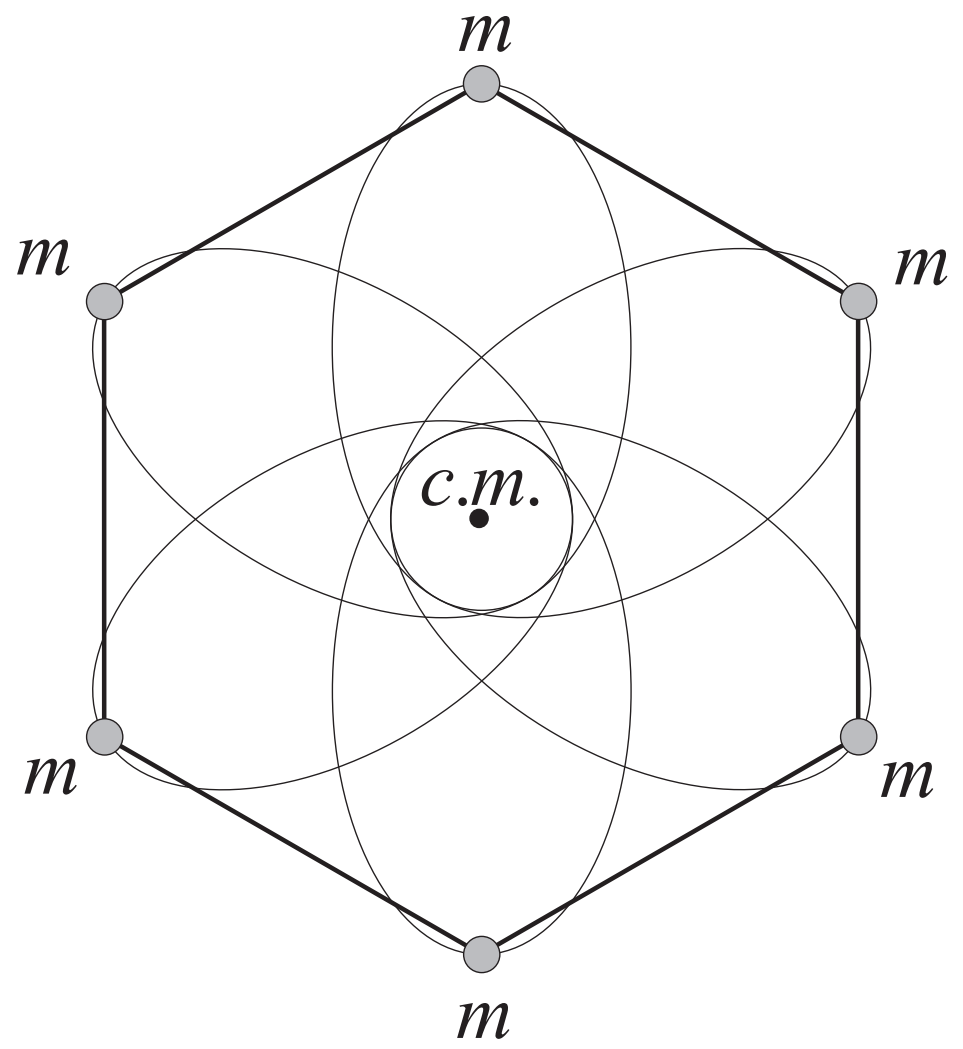

Figura 5.12: Solução homográfica para seis corpos. 


\section{Referências Bibliográficas}

[1] I. Newton, Philosophia Naturalis Principia Mathematica, Royal Society, London, 1687.

[2] H. BRuns, Über des integrales der Vielkörperproblem, Acta Mathematica, vol. 11, (1887).

[3] R. Abrahan and J. Marsden, Foundations of Mechanics, $2^{\text {nd }}$ edition, Adison Wesley, New York, (1994).

[4] F. Diacu, N-body Problem, Encyclopedia of Nonlinear Science, Fitzroy Dearbon (2003).

[5] M. Alonso E E. Finn, Física um Curso Universitário, vol. I,Edgard Bluscher, São Paulo (1972).

[6] N. CopéRnico, De revolutionibus orbium coelestium, (1543).

[7] R. Klippert, Programa mínimo em Cosmologia (Parte de Teoria da Relatividade Especial); Ainda não editado pelo ICRA/CBPF.

[8] E. Lifshitz, L. Landau, Mechanics, $3^{\text {rd }}$ edition, Butterworth-Heinemann, (1982).

[9] J. Moser, Regularization of Kepler's Problem and the Averaging Method on a Manifold, Comunications of pure and Applied Mathematics, vol. 23, (1970), 609-636.

[10] J.KePler, Astronomia nova, (1610).

[11] J.KePLER, Harmonice Mundi, (1614).

[12] G.G. Luther and W.R. Towler, Physical Review Letters vol. 48, 121 (1982).

[13] P. Smith, R. Smith, Mechanics; $2^{\text {nd }}$ edition, John Wiley \& Sons, New York, (1990).

[14] D.Figueiredo E A. Neves, Equações Diferenciais Aplicadas, Coleção Matemática Universitária - IMPA , Rio de Janeiro, (2005);

[15] W. Hamilton, The Hodograph or a new method of expressing in symbolic language the Newtonian law of attraction, Procedings of Royal Irish Academy, vol. 3, (1846), 344-353.

[16] J. Milnor, On the Geometry of Kepler Problem, The American Mathematical Monthly, vol. 90, (1983), 353-365.

[17] E. Belbruno, Two-Body motion under the inverse square central force and equivalent geodesic flows, Celestial Mechanics, vol. 15, (1977), 465-476. 
[18] W. Kaplan, Topology of the Two-Body Problem, American Mathematical Monthly, vol. 49, (1942), 316-323.

[19] H. Goldstein, C. Poole, J. Safko, Classical Mechanics, Addison Wesley; $3^{\text {th }}$, (2002).

[20] L. Euler, De moto rectilineo trium corporum se mutuo attahentium, Novi Comm. Acad. Sci. Imp. Petrop. vol. 11 (1767), 144-151.

[21] J.L. Lagrange, Essai sur le problème de trois corps, Ouvres, vol. 6, Gauthier-Villars, Paris, 1873.

[22] C. Moore, Braids in classical gravity, Physical Review Letters, vol. 70, (1993).

[23] A. Chenciner e R. Montgomery, A remarkable periodic solution for three-body problem in case of equal masses, Annals of Mathematics, vol. 152, (2000). 881-901.

[24] A. Chenciner, De l'espace des triangles au problème des 3 corps, Gazette des Math., mai (2004), 23-36.

[25] R. Moeckel, Some qualitative features of the three-body problem, Contemp. Math., vol. 81, (1988), 1-22.

[26] Kuo-Chang Chen, On Chenciner Montgomery's Orbit in the Three Body Problem, Discrete and Continuous Dynamical Systems, vol. 7, $\mathrm{n}^{\mathrm{o}} 1$ (2001), 85-90.

[27] W. Gordon W., A minimizing property of Kleperian orbits, Amer. J. Math, vol. 99, (1970).

[28] D. SAARI, Collisions, Rings and Other Newtonian N-Body Problems, CBMS, 104, (2005).

[29] R. Montgomery, Infinitely Many Syzygies Arch. Rat. Mech., vol. 164, no.4, 311-340, (2002).

[30] R. Moeckel, Shooting for the Eight - A Topological Existence Proof for a FigureEight Orbit of the Three-Body Problem, Rick Moeckel's home page: URL: http://www.math.umn.edu/ rick/research/Research.html (1/24/2007).

[31] J. Sotomayor, LIÇÕES DE EQUAÇÕES DIFERENCIAIS ORDINÁRIAS, Rio de Janeiro: IMPA - Projeto Euclides, (1979).

[32] E. Julliard-Toseal, Bruns' Theorem: the proof and some generalizations, Celestial Mechanics and Dynamical Astronomy, vol. 76, (2000), 241-281.

[33] S. Volchan, Uma Introdução à Mecânica Celeste, P. matemáticas - IMPA (2007).

[34] A. Wintner, Analytical Foundations of Celestial Mechanics, Princeton University Press, (1941).

[35] C. Vidal, G. Renildo, Homografic Solutions in the n-body problem. CUBO, vol. 6, 185$207,(2004)$. 
[36] D. SAARI, Collisions, Rings and Other N-Body Problems. CBMS, no. 104,(2000).

[37] S. Smale, Topology and Mechanics I, Inventiones Mathematicae, vol. 11, (1970), 4564.

[38] D. SAARI, On the role and properties of central configurations. Celestial Mechanics, vol. 21,9-20,(1980).

[39] C. Mccord, Planar central configuration estimates in the $N$-body problem, Ergodic Theory and Dynamical Systems, vol 16, 1059 - 1070, (1996).

[40] F. Moulton, An Introduction to Celestial Mechanics, Dover Publications, (1984).

[41] S. Smale, Mathematical problems for the next century, Math. Intelligencer, vol. 20, pp.7-15, (1998);

[42] M. Hampton, R. Moeckel, Finiteness of relative equilibria of the four-body problem, Invent. Math., vol. 163, 289-312, (2006).

[43] G. Roberts, A continuum of relative equilibria in the five-body problem, Physica D, vol. 127, 141-145, (1999).

[44] Y. Hagihara, Celestial Mechanics, vol 1, MIT Press, Massachusetts, (1970).

[45] J. Bernat, J. Llibre e E. Pérez-Chavela, On the planar central configurations of the 4-body problem with three equal masses, Dynam. Contin. Discrete Impuls. Systems, vol. 16, 1-13,(2009);

[46] L. Mello; F. CHAVES; A. C. Fernandes, Configurações Centrais Planares do Tipo Pipa. Revista Brasileira de Ensino de Física, v. 31, p. 1302, (2009);

[47] L.F. Mello e A.C. Fernandes, Configurações centrais planares encaixantes, Revista Brasileira de Ensino de Física 29, 385 (2007).

[48] L. F. Mello, A. C. Fernandes, New Spatial Central Configurations in the 5-body Problem, Submetido, (2009);

[49] E. S. Leandro, Finitness and Bifurcations of some Symmetrical Classes of Central Configurations, Arch. Ratio. Mech. Anal., vol. 167, 147-177, (2003);

[50] L. F. Mello, A. C. Fernandes, New Classes of Central Configurations in the Spatial 6-body Problem, Submetido, (2009);

[51] R. Moeckel, On central configurations, Math. Z., vol. 205 (1990), 499-517. 


\section{Índice Remissivo}

Órbita em Oito, 57

Aspectos geométricos, 22

Campo central, 6

Campo conservativo, 6

Centro de massa, 5

Classes de configurações centrais, 82

Configuração central, 81

Conjunto colisão, 72

Coordenadas generalizadas, 7

Corpo, 4

Energia cinética, 6

Energia mecânica, 7

Equações de Dziobek, 85

Equações de Euler-Lagrange, 9

Esfera de forma, 48

Lagrangiana, 8

Lei da gravitação de Newton, 12

Leis, 73

Leis de Kepler, 12

Leis de Newton, 5

Mínima ação, 8

massa, 5

Momento angular, 5

Momento angular total, 6

Momento conjugado, 9

Momento de Inércia, 6

Momento linear total, 5

Postulados de Newton, 4

Problema de $n$-corpos, 73

Regularização, 27
Solução de Chenciner e Montgomery, 48

Solução de equilíbrio relativo, 83

Solução de Euler, 41

Solução de Lagrange, 44

Solução homográfica, 82

Teorema de Bruns, 73

Velocidade, 4 